\title{
Second quantization method in the presence of bound states of particles
}

\author{
Sergey V. Peletminski* and Yuriy V. Slyusarenkd \\ National Science Center "Kharkov Institute of Physics and Technology" \\ Akademicheskaya Str.,1, Kharkov, 61108, Ukraine
}

(Dated: August 21, 2018)

\begin{abstract}
We develop an approximate second quantization method for describing the many-particle systems in the presence of bound states of particles at low energies (the kinetic energy of particles is small in comparison to the binding energy of compound particles). In this approximation the compound and elementary particles are considered on an equal basis. This means that creation and annihilation operators of compound particles can be introduced. The Hamiltonians, which specify the interactions between compound and elementary particles and between compound particles themselves are found in terms of the interaction amplitudes for elementary particles. The nonrelativistic quantum electrodynamics is developed for systems containing both elementary and compound particles. Some applications of this theory are considered.
\end{abstract}

PACS numbers: 03.65.Ca, 05.30.-d, 12.20.-m

*Electronic address: spelet@kipt.kharkov.ua

${ }^{\dagger}$ Electronic address: slusarenko@kipt.kharkov.ua 


\section{INTRODUCTION}

The second quantization is the effective method, which is used for description of physical processes in quantum theory of many-particle systems. The key role of this method consists in introduction of creation and annihilation operators of particles in a certain quantum state. The operators of physical quantities are constructed in terms of creation and annihilation operators. Such a description of quantum many-particle systems implies the particles to be elementary (not consisting of other particles). Moreover, it is absolutely accurate despite of the possible existence of compound particles. Since the interactions between particles may lead to formation of bound states, the standard second quantization method becomes too cumbersome. For this reason the construction of approximate quantummechanical theory for many-particle systems consisting of elementary particles and their bound states represents an actual problem. In this theory it is necessary to introduce the creation and annihilation operators of bound states as the operators of elementary objects (not compound). In addition we should preserve the required information concerning internal degrees of freedom for bound states. Such an approach may be actually realized in the approximation in which the binding energy of compound particles is much greater in comparison to their kinetic energy.

The problems leading to the necessity of accounting for compound particles along with elementary ones are typical in studying of the interaction of irradiation with matter (the matter consists of neutral atoms or molecules). The atoms or molecules may be both in the ground and excited states when it is necessary to take into account their internal structure retaining the convenience and simplicity of the second quantization method. Such a situation should take place in describing the experiments on laser cooling of atoms ${ }^{1-3}$ or experiments on observation of BoseEinstein condensates in magnetic traps, where a condensate temperature is reached by laser cooling of gases of alkali atoms $^{4}$. The similar problem of accounting for the bound states occurs also in quantum chemistry, for example, in studying of chemical reactions.

Thus, the construction of the quantum theory of many-particle systems in the presence of bound states of particles within the second quantization method represents not only academic but also applied interest. The formulation of the quantum theory itself is to be done so that the operators of physical quantities are constructed through the creation and annihilation operators of elementary particles and their bound states.

In this paper we demonstrate a possibility of construction of the above-mentioned quantum-mechanical theory by considering a system of two kinds of charged fermions. In this theory the compound particles (atoms) consist of two fermions of various kinds. The choice of such model is not associated with principle difficulties and makes it possible to simplify calculations and to obtain the visual results.

As applications of the developed theory we study a spontaneous radiation of two-component excited atom and obtain the expression for its probability (see, e.g., ${ }^{5}$ ). We also investigate the attractive forces acting between neutral atoms at long distances (the van der Waals forces) and derive the expression for the potential of the van der Waals forces ${ }^{6}$. Finally we consider a question concerning the scattering of photons and elementary particles by bound states ${ }^{7}$. 


\section{CREATION AND ANNIHILATION OPERATORS OF BOUND STATES OF PARTICLES}

Consider a system consisting of two kinds of fermions with masses $m_{1}$ and $m_{2}$. As was mentioned in the Introduction this case is more visual in order to demonstrate the procedure for obtaining the operators of basic physical quantities within the second quantization method in the presence of bound states of particles. We shall see that the developed methodology does not contain any difficulties for its generalization (the bound states of more than two particles, the presence of bosons in the system, the bound states of bosons and boson-fermion bound states). For simplicity we do not take into account a spin variable (this can be done without any difficulties).

Let $\hat{\psi}_{1}(\mathbf{x}), \hat{\psi}_{2}(\mathbf{x})$ be the annihilation operators of two kinds of fermions at the point $\mathbf{x}$,

$$
\hat{\psi}_{1}(\mathbf{x})|0\rangle=\hat{\psi}_{2}(\mathbf{x})|0\rangle=0
$$

where $|0\rangle$ is a vacuum state vector. Then the state vectors

$$
\left|\mathbf{x}_{1}, \ldots, \mathbf{x}_{n}, \mathbf{y}_{1}, \ldots, \mathbf{y}_{m}\right\rangle=\hat{\psi}_{1}^{+}\left(\mathbf{x}_{1}\right) \ldots \hat{\psi}_{1}^{+}\left(\mathbf{x}_{n}\right) \hat{\psi}_{2}^{+}\left(\mathbf{y}_{1}\right) \ldots \hat{\psi}_{2}^{+}\left(\mathbf{y}_{m}\right)|0\rangle
$$

$(n, m=0,1,2, \ldots)$ form a basis in the space of states $H$. In these states the particles are at the certain points $\mathbf{x}_{1}, \ldots, \mathbf{x}_{n} ; \mathbf{y}_{1}, \ldots, \mathbf{y}_{m} \in R$ of the coordinate space. The state vectors (1) satisfy the orthogonality and normalization relations and form a complete set of state vectors.

We assume that the particles of two different kinds form a bound state specified by the wave function

$$
\varphi_{\alpha}\left(\mathbf{x}_{1}, \mathbf{x}_{2}, \mathbf{x}\right)=\varphi_{\alpha}\left(\mathbf{x}_{1}-\mathbf{x}_{2}\right) \delta(\mathbf{x}-\mathbf{X}), \quad \mathbf{X}=\frac{m_{1} \mathbf{x}_{1}+m_{2} \mathbf{x}_{2}}{m_{1}+m_{2}}
$$

where $\mathbf{x}$ is the space coordinate and $\alpha$ are the quantum numbers of the bound state (atom) (we suppose that the particles of the same kind do not form the bound states). The corresponding state vector has the form

$$
|\alpha, \mathbf{x}\rangle=\int d \mathbf{x}_{1} d \mathbf{x}_{2} \varphi_{\alpha}\left(\mathbf{x}_{1}-\mathbf{x}_{2}\right) \delta(\mathbf{x}-\mathbf{X}) \hat{\psi}_{1}^{+}\left(\mathbf{x}_{1}\right) \hat{\psi}_{2}^{+}\left(\mathbf{x}_{2}\right)|0\rangle
$$

For this reason the operators

$$
\hat{\varphi}_{\alpha}^{+}(\mathbf{x})=\int d \mathbf{x}_{1} d \mathbf{x}_{2} \varphi_{\alpha}\left(\mathbf{x}_{1}-\mathbf{x}_{2}\right) \delta(\mathbf{x}-\mathbf{X}) \hat{\psi}_{1}^{+}\left(\mathbf{x}_{1}\right) \hat{\psi}_{2}^{+}\left(\mathbf{x}_{2}\right)
$$

we shall call as the creation operators of the bound states (atoms), so that

$$
\hat{\varphi}_{\alpha}^{+}(\mathbf{x})|0\rangle=|\alpha, \mathbf{x}\rangle, \quad \hat{\varphi}_{\alpha}(\mathbf{x})|0\rangle=0
$$

If the atom has a certain momentum, then its state vector is given by

$$
|\alpha, \mathbf{p}\rangle=\frac{1}{\sqrt{\mathcal{V}}} \int d \mathbf{x}_{1} d \mathbf{x}_{2} \varphi_{\alpha}\left(\mathbf{x}_{1}-\mathbf{x}_{2}\right) e^{i \mathbf{p} \mathbf{x}} \hat{\psi}_{1}^{+}\left(\mathbf{x}_{1}\right) \hat{\psi}_{2}^{+}\left(\mathbf{x}_{2}\right)|0\rangle
$$

where $\mathcal{V}$ is the volume of the system. The corresponding creation operator $\hat{\varphi}_{\alpha}^{+}(\mathbf{p})$ of the atom in the state with momentum $\mathbf{p}$ is defined by

$$
|\alpha, \mathbf{p}\rangle=\hat{\varphi}_{\alpha}^{+}(\mathbf{p})|0\rangle, \quad \hat{\varphi}_{\alpha}^{+}(\mathbf{x})=\frac{1}{\sqrt{\mathcal{V}}} \sum_{\mathbf{p}} \hat{\varphi}_{\alpha}^{+}(\mathbf{p}) e^{-i \mathbf{p x}} .
$$


Taking into account that

$$
\int d \mathbf{y}_{1} \varphi_{\alpha}^{*}\left(\mathbf{y}_{1}-\mathbf{y}_{2}\right) \varphi_{\beta}\left(\mathbf{y}_{1}-\mathbf{y}_{2}\right)=\delta_{\alpha \beta}
$$

it is easy to find the following commutation relations:

$$
\left[\hat{\varphi}_{\alpha}(\mathbf{x}), \hat{\varphi}_{\alpha^{\prime}}^{+}\left(\mathbf{x}^{\prime}\right)\right]=\delta_{\alpha \alpha^{\prime}} \delta\left(\mathbf{x}-\mathbf{x}^{\prime}\right)+\hat{\chi}_{\alpha \alpha^{\prime}}\left(\mathbf{x}, \mathbf{x}^{\prime}\right), \quad\left[\hat{\varphi}_{\alpha}(\mathbf{x}), \hat{\varphi}_{\alpha^{\prime}}\left(\mathbf{x}^{\prime}\right)\right]=0
$$

where

$$
\begin{aligned}
\hat{\chi}_{\alpha \alpha^{\prime}}\left(\mathbf{x}, \mathbf{x}^{\prime}\right)= & \int d \mathbf{y} d \mathbf{y}^{\prime} \varphi_{\alpha}^{*}(\mathbf{y}) \varphi_{\alpha \prime}\left(\mathbf{y}^{\prime}\right)\left\{\hat{\psi}_{1}^{+}\left(\mathbf{x}+\frac{m_{2}}{M} \mathbf{y}\right) \hat{\psi}_{1}\left(\mathbf{x}^{\prime}+\frac{m_{2}}{M} \mathbf{y}^{\prime}\right) \delta\left(\mathbf{y}-\mathbf{y}^{\prime}-\frac{m_{1}}{M}\left(\mathbf{x}-\mathbf{x}^{\prime}\right)\right)\right. \\
& \left.+\hat{\psi}_{2}^{+}\left(\mathbf{x}^{\prime}-\frac{m_{1}}{M} \mathbf{y}^{\prime}\right) \hat{\psi}_{2}\left(\mathbf{x}-\frac{m_{1}}{M} \mathbf{y}\right) \delta\left(\mathbf{y}-\mathbf{y}^{\prime}+\frac{m_{2}}{M}\left(\mathbf{x}-\mathbf{x}^{\prime}\right)\right)\right\}, \quad M=m_{1}+m_{2}
\end{aligned}
$$

moreover, $\hat{\chi}_{\alpha \alpha^{\prime}}\left(\mathbf{x}, \mathbf{x}^{\prime}\right)|0\rangle=0$. The vectors

$$
|\underbrace{\mathbf{x}_{1}, \ldots}_{n}, \underbrace{\mathbf{y}_{1}, \ldots}_{m}, \underbrace{\mathbf{z}_{1}, \ldots}_{l}\rangle \equiv \prod_{i=1}^{n} \hat{\psi}_{1}^{+}\left(\mathbf{x}_{i}\right) \prod_{k=1}^{m} \hat{\psi}_{2}^{+}\left(\mathbf{y}_{k}\right) \prod_{j=1}^{l} \hat{\varphi}_{\alpha_{j}}^{+}\left(\mathbf{z}_{j}\right)|0\rangle
$$

have an obvious physical meaning under the following conditions:

$$
\begin{aligned}
& \left|\mathbf{x}_{i}-\mathbf{x}_{j}\right| \gtrsim a, \quad\left|\mathbf{y}_{i}-\mathbf{y}_{j}\right| \gtrsim a, \quad\left|\mathbf{z}_{i}-\mathbf{z}_{j}\right| \gtrsim a, \\
& \left|\mathbf{x}_{i}-\mathbf{y}_{j}\right| \gtrsim a, \quad\left|\mathbf{x}_{i}-\mathbf{z}_{j}\right| \gtrsim a, \quad\left|\mathbf{y}_{i}-\mathbf{z}_{j}\right| \gtrsim a,
\end{aligned}
$$

$\left(\mathbf{x}, \mathbf{y}, \mathbf{z} \in R_{a}, a>>r_{0}, r_{0}\right.$ is the radius of the bound state; the definition of $a$ see below). In this case the elementary particles and their bound states are at the certain space points.

Notice that the state vectors (4) do not form a basis in the Gilbert space $H$ if (5) are valid. However, their linear span, which is a totality of the following vectors

$$
\sum_{n, m, l} \int \underbrace{\int d \mathbf{x}_{1} \ldots \int d \mathbf{y}_{1} \ldots \int d \mathbf{z}_{1} \ldots}_{R_{a}} C(\underbrace{\mathbf{x}_{1}, \ldots}_{n}, \underbrace{\mathbf{y}_{1}, \ldots}_{m}, \underbrace{\mathbf{z}_{1}, \ldots}_{l})|\underbrace{\mathbf{x}_{1}, \ldots}_{n}, \underbrace{\mathbf{y}_{1}, \ldots}_{m}, \underbrace{\mathbf{z}_{1}, \ldots}_{l}\rangle
$$

form a subspace $H_{a}$ of the space $H$. Let us show that the state vectors (4) [with conditions (5)] form an orthonormalized basis in the subspace $H_{a}$. To this end it is necessary to take into account that in calculating of the following vacuum averages:

$$
\left\langle 0\left|\hat{\psi}_{1}\left(\mathbf{x}_{1}\right) \ldots \hat{\psi}_{2}\left(\mathbf{x}_{2}\right) \ldots \hat{\varphi}_{\alpha}(\mathbf{x}) \hat{\varphi}_{\alpha^{\prime}}^{+}\left(\mathbf{x}^{\prime}\right) \ldots \hat{\psi}_{2}^{+}\left(\mathbf{x}_{2}^{\prime}\right) \ldots \hat{\psi}_{1}^{+}\left(\mathbf{x}_{1}^{\prime}\right) \ldots\right| 0\right\rangle
$$

we can use the Wick theorem with the following contractions:

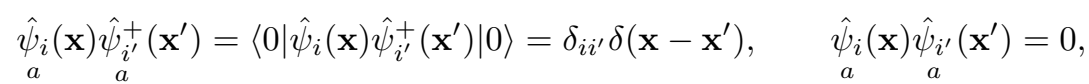

if to consider the operators $\hat{\psi}_{1}, \hat{\psi}_{2}, \hat{\varphi}$ referred to the moment of time +0 , and operators $\hat{\varphi}^{+}, \hat{\psi}_{2}^{+}, \hat{\psi}_{1}^{+}$to the moment of time -0 . In addition, we should remember that the creation and annihilation operators $\hat{\varphi}_{\alpha}, \hat{\varphi}_{\alpha^{\prime}}^{+}$depend on $\hat{\psi}_{i}, \hat{\psi}_{i^{\prime}}$ 
(see (3)). We also assume that the wave function (2) of the atom differs from zero for $\left|\mathbf{x}_{1}-\mathbf{x}_{2}\right|<r_{0}$. Taking into account (3) and noting that for $\left|\mathbf{x}_{1}^{\prime}-\mathbf{x}_{2}^{\prime}\right|>a$

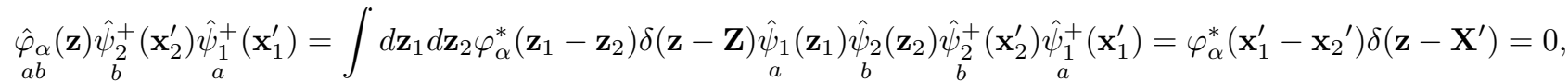

$$
\begin{aligned}
& \mathbf{X}^{\prime}=\frac{m_{1} \mathbf{x}_{1}^{\prime}+m_{2} \mathbf{x}_{2}^{\prime}}{m_{1}+m_{2}}
\end{aligned}
$$

and also

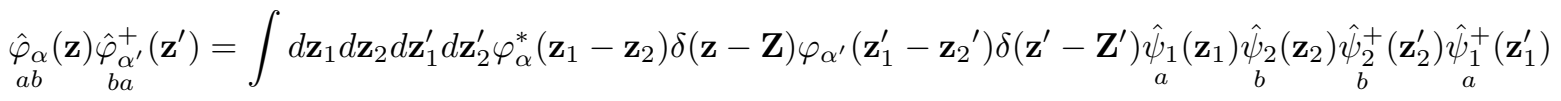

$$
\begin{aligned}
& =\int d \mathbf{z}_{1} d \mathbf{z}_{2} \varphi_{\alpha}^{*}\left(\mathbf{z}_{1}-\mathbf{z}_{2}\right) \delta(\mathbf{z}-\mathbf{Z}) \varphi_{\alpha^{\prime}}\left(\mathbf{z}_{1}-\mathbf{z}_{2}\right) \delta\left(\mathbf{z}^{\prime}-\mathbf{Z}\right)=\delta_{\alpha \alpha^{\prime}} \delta\left(\mathbf{z}-\mathbf{z}^{\prime}\right)
\end{aligned}
$$

(the double contractions correspond to the operators $\hat{\varphi}_{\alpha}, \hat{\varphi}_{\alpha}^{+}$) we get

$$
\begin{array}{r}
\left\langle 0|\underbrace{\hat{\psi}_{1}\left(\mathbf{x}_{1}\right) \ldots}_{n} \underbrace{\hat{\psi}_{2}\left(\mathbf{y}_{1}\right) \ldots}_{m} \underbrace{\hat{\varphi}_{\alpha_{1}}\left(\mathbf{z}_{1}\right) \ldots}_{l} \underbrace{\hat{\varphi}_{\alpha_{1}^{\prime}}^{+}\left(\mathbf{z}_{1}^{\prime}\right) \ldots}_{l^{\prime}} \underbrace{\hat{\psi}_{2}^{+}\left(\mathbf{y}_{1}^{\prime}\right) \ldots}_{m^{\prime}} \underbrace{\hat{\psi}_{1}\left(\mathbf{x}_{1}^{\prime}\right) \ldots}_{n^{\prime}}| 0\right\rangle= \\
=\delta_{n n^{\prime}} \delta_{m m^{\prime}} \delta_{l l^{\prime}} \sum \mathcal{P}_{x^{\prime}} \mathcal{P}_{y^{\prime}} \underbrace{\delta\left(\mathbf{x}_{1}-\mathbf{x}_{1}^{\prime}\right) \ldots}_{n} \underbrace{\delta\left(\mathbf{y}-\mathbf{y}_{1}^{\prime}\right) \ldots}_{m} \underbrace{\delta\left(\mathbf{z}_{1}-\mathbf{z}_{1}^{\prime}\right) \delta_{\alpha_{1} \alpha_{1}^{\prime}} \ldots}_{m}
\end{array}
$$

This relation shows that the vectors (6) form the orthonormalized basis in the subspace $H_{a}$ if to consider the creation and annihilation operators $\hat{\varphi}_{\alpha}^{+}(\mathbf{z}), \hat{\varphi}_{\alpha}(\mathbf{z})$ as the Bose operators, which commute with $\hat{\psi}_{i}(\mathbf{x}), \hat{\psi}_{i}^{+}(\mathbf{x})$. The quantity $\mathcal{P}_{x^{\prime}}$ in (7) is equal to +1 if the number of permutations of the arguments $\mathbf{x}_{1}^{\prime} \ldots \mathbf{x}_{n}^{\prime}$ is even and it is equal to -1 if the number of these permutations is odd. The quantity $\mathcal{P}_{y^{\prime}}$ is defined similarly. However, the case when it is necessary to take into account a more complicated arrangement of the contractions between $\varphi_{\alpha}, \varphi_{\alpha}^{+}$:

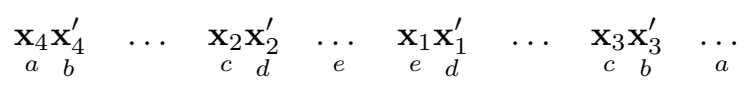

can take place (the odd indices correspond to the creation operators and even to annihilation operators). Since $\mathrm{x}_{1}^{\prime}=\mathrm{x}_{2}^{\prime}, \mathrm{x}_{2}=\mathrm{x}_{3}, \mathrm{x}_{4}^{\prime}=\mathrm{x}_{3}^{\prime}$

$$
\left|\mathbf{x}_{1}-\mathbf{x}_{1}^{\prime}\right| \lesssim r_{0}, \quad\left|\mathbf{x}_{1}^{\prime}-\mathbf{x}_{2}\right| \lesssim r_{0}, \quad\left|\mathbf{x}_{3}^{\prime}-\mathbf{x}_{2}\right| \lesssim r_{0}, \quad\left|\mathbf{x}_{4}-\mathbf{x}_{3}^{\prime}\right| \lesssim r_{0},
$$

whence $\left|\mathbf{x}_{4}-\mathbf{x}_{1}\right| \lesssim 4 r_{0}$. Therefore, if $a \leq 4 r_{0}$, then the above contractions are to be taken into account. If however, we consider the $n$-particle states, then the discussed arrangements of the contractions are not to be taken into account for $n \lesssim a / r_{0}$ (we emphasize that $r_{0} / a$ is a small parameter in our problem, see (11)). The said above concerns to the majority of problems in non-relativistic quantum theory, where a finite number of particles is usually studied (see sections $\mathrm{V}, \mathrm{VI})$.

With the use of (7) it is easy to find the projection operator $\mathcal{P}_{H_{a}}$ onto the subspace $H_{a}$ :

$$
\mathcal{P}_{H_{a}}=\sum_{k+m+l \leq n} \frac{1}{k !} \frac{1}{m !} \frac{1}{l !} \underbrace{\int d \mathbf{x}_{1} \ldots \int d \mathbf{y}_{1} \ldots \int d \mathbf{z}_{1} \ldots}_{R_{a}}|\underbrace{\mathbf{x}_{1} \ldots}_{k} \underbrace{\mathbf{y}_{1} \ldots}_{m} \underbrace{\mathbf{z}_{1} \ldots}_{l}\rangle\langle\underbrace{\mathbf{x}_{1} \ldots}_{k} \underbrace{\mathbf{y}_{1} \ldots}_{m} \underbrace{\mathbf{z}_{1} \ldots}_{l}|,
$$


such that the operators $\underline{A}$ acting in the subspace $H_{a} \in H$ correspond to the operators of physical quantities $A$ that act in $H$ (hereinafter the sums of $1,2, \ldots n$-particle subspaces over the bound states are considered; $n \approx a / r_{0}>>1$ ),

$$
\underline{A}=\mathcal{P}_{H_{a}} A \mathcal{P}_{H_{a}} .
$$

Let us introduce now an auxiliary space $\tilde{H}$ with the Fermi creation and annihilation operators $\hat{\chi}_{1}^{+}(\mathbf{x}), \hat{\chi}_{2}^{+}(\mathbf{x})$, $\hat{\chi}_{1}(\mathbf{x}), \hat{\chi}_{2}(\mathbf{x})$ and Bose creation and annihilation operators $\hat{\eta}_{\alpha}^{+}(\mathbf{x}), \hat{\eta}_{\alpha}(\mathbf{x})$ and take the vectors

$$
\left.\left.\mid \mathbf{x}_{1}, \ldots, \mathbf{y}_{1}, \ldots, \mathbf{z}_{1}, \ldots\right)=\hat{\chi}_{1}^{+}\left(\mathbf{x}_{1}\right) \ldots \hat{\chi}_{2}^{+}\left(\mathbf{y}_{1}\right) \ldots \hat{\eta}_{\alpha}^{+}\left(\mathbf{z}_{1}\right) \ldots \mid 0\right)
$$

as a basis of this space, where $\mid 0)$ is a vacuum vector in $\tilde{H}$. Then the linear span of the vectors

$$
\left.\mid \mathbf{x}_{1}, \ldots, \mathbf{y}_{1}, \ldots, \mathbf{z}_{1}, \ldots\right) \in \tilde{H}_{a}, \quad \mathbf{x}, \mathbf{y}, \mathbf{z} \in R_{a}
$$

determines the subspace $\tilde{H}_{a}$ of the space $\tilde{H}$.

Now we can easily establish the isomorphic correspondence between $H_{a}$ and $\tilde{H}_{a}$ :

$$
\left.\left|\mathbf{x}_{1}, \ldots, \mathbf{y}_{1}, \ldots, \mathbf{z}_{1}, \ldots\right\rangle \Longleftrightarrow \mid \mathbf{x}_{1}, \ldots, \mathbf{y}_{1}, \ldots, \mathbf{z}_{1}, \ldots\right)
$$

which preserves the scalar product

$$
\left\langle\mathbf{x}^{\prime}, \ldots, \mathbf{y}^{\prime}, \ldots, \mathbf{z}^{\prime}, \ldots \mid \mathbf{x}, \ldots, \mathbf{y}, \ldots, \mathbf{z} \ldots\right\rangle=\left(\mathbf{x}^{\prime}, \ldots, \mathbf{y}^{\prime}, \ldots, \mathbf{z}^{\prime}, \ldots \mid \mathbf{x}, \ldots, \mathbf{y}, \ldots, \mathbf{z} \ldots\right), \quad \mathbf{x}, \mathbf{y}, \mathbf{z} ; \mathbf{x}^{\prime}, \mathbf{y}^{\prime}, \mathbf{z}^{\prime} \in R_{a}
$$

We can also establish the isomorphism between the operators $\underline{A} \Longleftrightarrow \tilde{A}$, acting in the spaces $H_{a}$ and $\tilde{H}_{a}$ according to the formula:

$$
\left\langle\mathbf{x}^{\prime}, \ldots, \mathbf{y}^{\prime}, \ldots, \mathbf{z}^{\prime}, \ldots|\underline{A}| \mathbf{x}, \ldots, \mathbf{y}, \ldots, \mathbf{z} \ldots\right\rangle=\left(\mathbf{x}^{\prime}, \ldots, \mathbf{y}^{\prime}, \ldots, \mathbf{z}^{\prime}, \ldots|\tilde{A}| \mathbf{x}, \ldots, \mathbf{y}, \ldots, \mathbf{z} \ldots\right)
$$

This isomorphic correspondence is remained after the multiplication of the operator by a number, after addition of operators, and after multiplication of operators:

$$
\lambda \underline{A} \Longleftrightarrow \lambda \tilde{A}, \quad \underline{A}+\underline{B} \Longleftrightarrow \tilde{A}+\tilde{B}, \quad \underline{A} \underline{B} \Longleftrightarrow \tilde{A} \tilde{B} .
$$

The formulas (8), (9) lead to

$$
\left(\mathbf{x}^{\prime}, \ldots, \mathbf{y}^{\prime}, \ldots, \mathbf{z}^{\prime}, \ldots|\tilde{A}| \mathbf{x}, \ldots, \mathbf{y}, \ldots, \mathbf{z} \ldots\right)=\left\langle\mathbf{x}^{\prime}, \ldots, \mathbf{y}^{\prime}, \ldots, \mathbf{z}^{\prime}, \ldots|A| \mathbf{x}, \ldots, \mathbf{y}, \ldots, \mathbf{z} \ldots\right\rangle
$$

This relation determines the operators of various physical quantities $\tilde{A}$ acting in $\tilde{H}_{a}$ and, hence, transfers the quantum theory in which the compound (the bound states) and elementary particles exist on an equal basis from the space of states $H$ onto the space of states $\tilde{H}_{a}$. We would like to remind here that $\hat{\varphi}_{\alpha}^{+}$entering (4) is determined by (3).

The relation (10) defines the operator $\tilde{A}$ in $\tilde{H}_{a}$ uniquely, but it does not define it uniquely in $\tilde{H}$. It is evident that the operator $\tilde{A}$ acting in $\tilde{H}$ (continued from $\tilde{H}_{a}$ to the whole space $\tilde{H}$ ) is determined up to the term $\tilde{A}^{\prime}$, the matrix elements of which are zero in the space $\tilde{H}_{a}\left(\tilde{A}=\tilde{A}^{\prime}+\tilde{A}^{\prime \prime}\right)$. If to introduce the projection operator $\mathcal{P}_{\tilde{H}_{a}}$ onto the subspace $\tilde{H}_{a}$ and to require that the operator $\tilde{A}$ has no nonzero matrix elements in the orthogonal subspace, then 
the operator $\tilde{A}$ will be defined in $\tilde{H}$ uniquely, $\tilde{A}=\mathcal{P}_{\tilde{H}_{a}} \tilde{A}^{\prime \prime} \mathcal{P}_{\tilde{H}_{a}}$. We will omit the projection operator $\mathcal{P}_{\tilde{H}_{a}}$ when constructing the operator $\tilde{A}$ acting in $\tilde{H}$. The reason for this is the assumption that the matrix elements of the operators (in the position space), corresponding to a quite large external parameter $R \sim\left|\mathbf{x}_{i}-\mathbf{x}_{j}\right|$ give a dominant contribution to the quantum-mechanical processes. Further we will assume that $R>>r_{0}$ (usually in case of particle collisions $R^{-1} \sim \sqrt{m \mathcal{E}}$, where $\mathcal{E}$ is the particle kinetic energy). The latter inequality makes it possible to choose parameter $a$ (see (5)) as follows ${ }^{10}$ :

$$
R>>a>>r_{0} .
$$

The matrix elements of the operators should not depend on parameter $a$ chosen by this way. Let us mentally decrease the radius of a bound state, $r_{0} \rightarrow 0$. Then the whole written scheme, as we have already noted, does not depend on $a$ up to the values $a=r_{0}$. Therefore, the subspace $\tilde{H}_{a}$ can be identified with $\tilde{H}$ due to the inequality $R>>a$. In other words one can considers that $\mathcal{P}_{\tilde{H}_{a}} \rightarrow 1$ for $a \rightarrow 0$. At the same time we do not break the quantum-mechanical description of bound states in virtue of the inequality $a>>r_{0}$. From the physical point of view the inequality $r_{0}<<R$ gives the stability domain for the bound states considered as elementary particles. The calculation of the following approximation is to be associated with accounting of the difference of the subspace $H_{a}$ from the space $H$.

Finally we note that for an arbitrary vector $\mid) \in \tilde{H}_{a}$ the following evident relations are valid:

$$
\left.\hat{\zeta}(\mathbf{x}) \hat{\xi}\left(\mathbf{x}^{\prime}\right) \mid\right)=0, \quad\left(\left|\hat{\zeta}^{+}(\mathbf{x}) \hat{\xi}^{+}\left(\mathbf{x}^{\prime}\right)=0, \quad\right| \mathbf{x}-\mathbf{x}^{\prime} \mid<a,\right.
$$

or

$$
\hat{\zeta}(\mathbf{x}) \hat{\xi}\left(\mathbf{x}^{\prime}\right) \tilde{H}_{a}=0, \quad \tilde{H}_{a} \hat{\zeta}^{+}(\mathbf{x}) \hat{\xi}^{+}\left(\mathbf{x}^{\prime}\right)=0, \quad\left|\mathbf{x}-\mathbf{x}^{\prime}\right|<a
$$

where $\hat{\zeta}$ and $\hat{\xi}$ are any of the annihilation operators $\hat{\chi}_{1}, \hat{\chi}_{2}, \hat{\eta}$.

\section{THE STRUCTURE OF $\tilde{\hat{A}}(\mathbf{u}, \mathbf{v})$ OPERATORS}

Here we consider the method for obtaining the operators $\tilde{\hat{A}}$. Let the operator $\hat{A}$ represents a normal-ordered production of $\hat{\psi}_{i}(\mathbf{v}), \hat{\psi}_{i}^{+}(\mathbf{u}),(i=1,2)$ :

$$
\hat{A}(\mathbf{u}, \mathbf{v})=\hat{\psi}_{1}^{+}\left(\mathbf{u}_{1}\right) \ldots \hat{\psi}_{2}^{+}\left(\mathbf{u}_{2}\right) \hat{\psi}_{1}\left(\mathbf{v}_{1}\right) \ldots \hat{\psi}_{2}\left(\mathbf{v}_{2}\right) \ldots
$$

The operators of such type are the particle density operator $\hat{\rho}_{i}(\mathbf{x})$,

$$
\hat{\rho}_{i}(\mathbf{x})=\hat{\psi}_{i}^{+}(\mathbf{x}) \hat{\psi}_{i}(\mathbf{x})
$$

the momentum density operator $\hat{\pi}_{i}(\mathbf{x})$,

$$
\hat{\boldsymbol{\pi}}_{i}(\mathbf{x})=-\frac{i}{2}\left(\hat{\psi}_{i}^{+}(\mathbf{x}) \frac{\partial \hat{\psi}_{i}(\mathbf{x})}{\partial \mathbf{x}}-\frac{\partial \hat{\psi}_{i}^{+}(\mathbf{x})}{\partial \mathbf{x}} \hat{\psi}_{i}(\mathbf{x})\right),
$$

a Hamiltonian of the system, etc. 
The matrix element in the right-hand side of (10) may be written as the following vacuum average:

$$
\left\langle 0\left|\hat{\psi}_{1}\left(\mathbf{x}_{1}\right) \ldots \hat{\psi}_{2}\left(\mathbf{x}_{2}\right) \ldots \hat{\varphi}_{\alpha}(\mathbf{x}) \ldots \hat{A}(\mathbf{u}, \mathbf{v}) \hat{\varphi}_{\alpha^{\prime}}^{+}(\mathbf{x}) \ldots \hat{\psi}_{2}\left(\mathbf{x}_{2}^{\prime}\right) \ldots \hat{\psi}_{1}\left(\mathbf{x}_{1}^{\prime}\right) \ldots\right| 0\right\rangle
$$

Let us note that when calculating this average by using Wick's theorem, the quantity, which is averaged over the vacuum state has the meaning of a mixed $T$-product if to consider the operators $\hat{\psi}_{1}, \ldots, \hat{\psi}_{2}, \ldots, \hat{\varphi} \ldots$ referring to the moment of time +0 , the operators $\hat{\psi}_{1}^{+}, \ldots, \hat{\psi}_{2}^{+}, \ldots, \hat{\varphi}^{+} \ldots$ to -0 , and the normal-ordered operator $\hat{A}(\mathbf{u}, \mathbf{v})$ refereing to the moment of time 0 . Thus, there is no need to place the contractions inside the expression for $\hat{A}(\mathbf{u}, \mathbf{v})$. Let

$$
A_{b}\left(\mathbf{y} ; \mathbf{y}^{\prime} ; \mathbf{u}, \mathbf{v}\right) \equiv A_{b}\left(\mathbf{y}_{1}, \ldots, \mathbf{y}_{2}, \ldots, \mathbf{y}, \ldots ; \mathbf{y}_{1}^{\prime}, \ldots, \mathbf{y}_{2}^{\prime}, \ldots, \mathbf{y}^{\prime}, \ldots ; \mathbf{u}, \mathbf{v}\right)
$$

be the analytic expression that corresponds to the diagram " $b$ ". For this diagram the operators with the arguments $\mathbf{u}$ are related to the operators with the arguments $\mathbf{y}_{1}, \ldots, \mathbf{y}_{2}, \ldots, \mathbf{y}, \ldots$ In addition, the latter arguments are spaced apart by the distances greater than $a$ and coincide with some of the arguments $\mathbf{x}_{1}, \ldots, \mathbf{x}_{2}, \ldots, \mathbf{x}, \ldots$, . The similar statement should be also made concerning the arguments $\mathbf{y}_{1}^{\prime}, \ldots, \mathbf{y}_{2}^{\prime}, \ldots, \mathbf{y}^{\prime}, \ldots$ Then, the operator $\tilde{\hat{A}}(\mathbf{u}, \mathbf{v})$ acting in $\tilde{H}$ is given, according to (5), by

$$
\tilde{\hat{A}}(\mathbf{u}, \mathbf{v})=\sum_{b} \int \hat{R}_{1} \hat{R}_{2} \hat{R} A_{b}\left(\mathbf{y} ; \mathbf{y}^{\prime} ; \mathbf{u}, \mathbf{v}\right) \hat{R}_{1}^{\prime} \hat{R}_{2}^{\prime} \hat{R}^{\prime}
$$

where

$$
\begin{aligned}
& \hat{R}_{1}=\prod \hat{\chi}^{+}\left(\mathbf{y}_{1}\right) d \mathbf{y}_{1}, \quad \hat{R}_{2}=\prod \hat{\chi}^{+}\left(\mathbf{y}_{2}\right) d \mathbf{y}_{2}, \quad \hat{R}=\prod \hat{\eta}^{+}(\mathbf{y}) d \mathbf{y}, \\
& \hat{R}_{1}^{\prime}=\prod \hat{\chi}\left(\mathbf{y}_{1}^{\prime}\right) d \mathbf{y}_{1}^{\prime}, \quad \hat{R}_{2}^{\prime}=\prod \hat{\chi}\left(\mathbf{y}_{2}^{\prime}\right) d \mathbf{y}_{2}^{\prime}, \quad \hat{R}^{\prime}=\prod \hat{\eta}\left(\mathbf{y}^{\prime}\right) d \mathbf{y}^{\prime}
\end{aligned}
$$

Here the summation is taken over all diagrams of the described type.

If $\hat{A}(\mathbf{v})=1$ (see the proof of (7) $)$, then $\tilde{\hat{A}}(\mathbf{v})=1$ on the subspace $\tilde{H}_{a}$. Let now $\hat{A}(\mathbf{u}, \mathbf{v})=\hat{\psi}_{1}(\mathbf{v})$. Then the only diagrams of the described type for the vacuum average $\left\langle 0\left|\ldots \hat{\psi}_{1}(\mathbf{v}) \ldots\right| 0\right\rangle$ are the following diagrams:

$$
A_{b_{1}}=\hat{\psi}_{a}(\mathbf{v}) \ldots \hat{\psi}_{a}^{+}\left(\mathbf{y}^{\prime}\right), \quad A_{b_{2}}=\underset{a}{\hat{\psi}_{2}}\left(\mathbf{y}_{2}\right) \ldots \hat{\psi}_{b}(\mathbf{v}) \ldots \underset{b a}{\hat{\varphi}_{\alpha}^{+}}\left(\mathbf{y}^{\prime}\right) .
$$

The expressions

$$
\begin{gathered}
A_{b_{1}}\left(\mathbf{y}_{1}^{\prime} ; \mathbf{v}\right)=\delta\left(\mathbf{v}-\mathbf{y}_{1}^{\prime}\right) \\
A_{b_{2}}\left(\mathbf{y}_{2} ; \mathbf{y}^{\prime}, \mathbf{v}\right)=\int d \mathbf{x}_{1} d \mathbf{x}_{2} \varphi_{\alpha}\left(\mathbf{x}_{1}-\mathbf{x}_{2}\right) \delta\left(\mathbf{y}^{\prime}-\mathbf{X}\right) \delta\left(\mathbf{v}-\mathbf{x}_{1}\right) \delta\left(\mathbf{x}_{2}-\mathbf{y}_{2}\right)=\varphi_{\alpha}\left(\mathbf{v}, \mathbf{y}_{2}, \mathbf{y}^{\prime}\right),
\end{gathered}
$$

correspond to the above mentioned diagrams. Here $\varphi_{\alpha}\left(\mathbf{v}, \mathbf{y}_{2}, \mathbf{y}^{\prime}\right)$ is defined in accordance with (2). Therefore, according to (15) we have

$$
\tilde{\hat{\psi}}_{1}(\mathbf{v})=\int d \mathbf{y}_{1}^{\prime} A_{b_{1}}\left(\mathbf{y}_{1}^{\prime}, \mathbf{v}\right) \hat{\chi}_{1}\left(\mathbf{y}_{1}^{\prime}\right)+\int d \mathbf{y}_{2} d \mathbf{y}^{\prime} A_{b_{2}}\left(\mathbf{y}_{2} ; \mathbf{y}^{\prime}, \mathbf{v}\right) \hat{\chi}_{2}^{+}\left(\mathbf{y}_{2}\right) \hat{\eta}\left(\mathbf{y}^{\prime}\right)=\hat{\chi}_{1}(\mathbf{v})+\hat{O}_{1}(\mathbf{v}),
$$

where

$$
\hat{O}_{1}(\mathbf{v})=\int d \mathbf{y} \hat{\varphi}(\mathbf{v}, \mathbf{y}) \hat{\chi}_{2}^{+}(\mathbf{y}), \quad \hat{\varphi}\left(\mathbf{x}_{1}, \mathbf{x}_{2}\right)=\varphi_{\alpha}(\mathbf{x}) \hat{\eta}_{\alpha}(\mathbf{X})
$$


and $\mathbf{X}$ is given by (2), $\mathbf{x}=\mathbf{x}_{1}-\mathbf{x}_{2}$. Similarly, one finds

$$
\tilde{\hat{\psi}}_{2}(\mathbf{v})=\hat{\chi}_{2}(\mathbf{v})+\hat{O}_{2}(\mathbf{v})
$$

where

$$
\hat{O}_{2}(\mathbf{v})=\int d \mathbf{y} \hat{\chi}_{1}^{+}(\mathbf{y}) \hat{\varphi}(\mathbf{y}, \mathbf{v})
$$

When deriving (16), (18), we have essentially used the inequalities (5).

Now let us consider $\hat{A}(\mathbf{u}, \mathbf{v})=\hat{\psi}_{1}^{+}(\mathbf{u}) \hat{\psi}_{1}(\mathbf{v})$. In this case the following five diagrams

$$
\begin{aligned}
& A_{1}=\hat{\psi}_{a} \ldots \hat{\psi}_{a}^{+}(\mathbf{u}) \hat{\psi}_{b}(\mathbf{v}) \ldots \hat{\psi}_{b}^{+}, \quad A_{2}=\underset{a}{\hat{\psi}} \ldots \hat{\psi}_{b} \ldots \hat{\psi}_{b}^{+}(\mathbf{u}) \hat{\psi}_{c}(\mathbf{v}) \ldots \hat{\varphi}_{c a}^{+}, \quad A_{3}=\underset{a b}{\hat{\varphi}} \ldots \hat{\psi}_{b}^{+}(\mathbf{u}) \hat{\psi}_{c}(\mathbf{v}) \ldots \hat{\psi}_{c}^{+} \ldots \hat{\psi}_{a}^{+}, \\
& A_{4}=\underset{a b}{\hat{\varphi}} \ldots \hat{\psi}_{b}^{+}(\mathbf{u}) \hat{\psi}_{c}(\mathbf{v}) \ldots \underset{c a}{\hat{\varphi}_{c}^{+},} \quad A_{5}=\underset{a}{\hat{\psi}} \ldots \underset{b d}{\hat{\varphi}} \ldots \hat{\psi}_{d}^{+}(\mathbf{u}) \hat{\psi}_{c}(\mathbf{v}) \ldots \hat{\varphi}_{c a}^{+} \ldots \hat{\psi}_{b}^{+}
\end{aligned}
$$

correspond to this operator (the indices 1 and 2 for $\hat{\psi}$ and $\hat{\psi}^{+}$can be easy restored if to take into account (3) and the definition of contractions). The analytic expressions for these diagrams have the form

$$
\begin{gathered}
\tilde{\hat{A}}_{1}=\hat{\chi}_{1}^{+}\left(\mathbf{u}_{1}\right) \hat{\chi}_{1}\left(\mathbf{v}_{1}\right), \quad \tilde{\hat{A}}_{2}=\int d \mathbf{z}_{1} d \mathbf{z}_{2} \hat{\varphi}\left(\mathbf{z}_{1}, \mathbf{z}_{2}\right) \delta\left(\mathbf{z}_{1}-\mathbf{v}_{1}\right) \hat{\chi}_{1}^{+}\left(\mathbf{u}_{1}\right) \hat{\chi}_{2}^{+}\left(\mathbf{z}_{2}\right)=\int d \mathbf{z}_{2} \hat{\varphi}\left(\mathbf{v}_{1}, \mathbf{z}_{2}\right) \hat{\chi}_{1}^{+}\left(\mathbf{u}_{1}\right) \hat{\chi}_{2}^{+}\left(\mathbf{z}_{2}\right), \\
\tilde{\hat{A}}_{3}=\int d \mathbf{z}_{1} d \mathbf{z}_{2} \hat{\varphi}^{+}\left(\mathbf{z}_{1}, \mathbf{z}_{2}\right) \delta\left(\mathbf{z}_{1}-\mathbf{u}_{1}\right) \hat{\chi}_{2}\left(\mathbf{z}_{2}\right) \hat{\chi}_{1}\left(\mathbf{v}_{1}\right)=\int d \mathbf{z}_{2} \hat{\varphi}^{+}\left(\mathbf{u}_{1}, \mathbf{z}_{2}\right) \hat{\chi}_{2}\left(\mathbf{z}_{2}\right) \hat{\chi}_{1}\left(\mathbf{v}_{1}\right), \\
\tilde{\hat{A}}_{4}=\int d \mathbf{z}_{1} d \mathbf{z}_{2} d \mathbf{z}_{1}^{\prime} d \mathbf{z}_{2}^{\prime} \hat{\varphi}^{+}\left(\mathbf{z}_{1}, \mathbf{z}_{2}\right) \hat{\varphi}\left(\mathbf{z}_{1}^{\prime}, \mathbf{z}_{2}^{\prime}\right) \delta\left(\mathbf{z}_{1}-\mathbf{u}_{1}\right) \delta\left(\mathbf{v}_{1}-\mathbf{z}_{1}^{\prime}\right) \delta\left(\mathbf{z}_{2}-\mathbf{z}_{2}^{\prime}\right)=\int d \mathbf{z}_{2} \hat{\varphi}^{+}\left(\mathbf{u}_{1}, \mathbf{z}_{2}\right) \hat{\varphi}\left(\mathbf{v}_{1}, \mathbf{z}_{2}\right), \\
\tilde{\hat{A}}_{5}=-\int d \mathbf{z}_{1} d \mathbf{z}_{2} d \mathbf{z}_{1}^{\prime} d \mathbf{z}_{2}^{\prime} \hat{\varphi}^{+}\left(\mathbf{z}_{1}, \mathbf{z}_{2}\right) \hat{\varphi}\left(\mathbf{z}_{1}^{\prime}, \mathbf{z}_{2}^{\prime}\right) \delta\left(\mathbf{z}_{1}-\mathbf{u}_{1}\right) \delta\left(\mathbf{v}_{1}-\mathbf{z}_{1}^{\prime}\right) \hat{\chi}_{2}^{+}\left(\mathbf{z}_{2}^{\prime}\right) \hat{\chi}_{2}\left(\mathbf{z}_{2}\right) \\
=-\int d \mathbf{z}_{2} d \mathbf{z}_{2}^{\prime} \hat{\varphi}^{+}\left(\mathbf{u}_{1}, \mathbf{z}_{2}\right) \hat{\varphi}\left(\mathbf{v}_{1}, \mathbf{z}_{2}^{\prime}\right) \hat{\chi}_{2}^{+}\left(\mathbf{z}_{2}^{\prime}\right) \hat{\chi}_{2}\left(\mathbf{z}_{2}\right),
\end{gathered}
$$

whence, we find the operators (15) corresponding to the diagrams (14),

$$
\tilde{\hat{A}}_{1}=\hat{\chi}_{1}^{+}\left(\mathbf{u}_{1}\right) \hat{\chi}_{1}\left(\mathbf{v}_{1}\right), \quad \tilde{\hat{A}}_{2}=\hat{\chi}_{1}^{+}\left(\mathbf{u}_{1}\right) \hat{O}_{1}\left(\mathbf{v}_{1}\right), \quad \tilde{\hat{A}}_{3}=\hat{O}_{1}^{+}\left(\mathbf{u}_{1}\right) \hat{\chi}_{1}\left(\mathbf{v}_{1}\right), \quad \tilde{\hat{A}}_{4}+\tilde{\hat{A}}_{5}=\hat{O}_{1}^{+}\left(\mathbf{u}_{1}\right) \hat{O}_{1}\left(\mathbf{v}_{1}\right) .
$$

In obtaining the latter expression, we have taken into account the anticommutative relations for $\hat{\chi}, \hat{\chi}^{+}$. Next bearing in mind (16), (18) we obtain the final expression for $\tilde{\hat{A}}(\mathbf{u}, \mathbf{v})=\hat{\psi}_{1}^{+} \widetilde{\left(\mathbf{u}_{1}\right) \hat{\psi}_{1}}\left(\mathbf{v}_{1}\right)$ that corresponds to $\hat{A}(\mathbf{u}, \mathbf{v})=\hat{\psi}_{1}^{+}\left(\mathbf{u}_{1}\right) \hat{\psi}_{1}\left(\mathbf{v}_{1}\right)$ :

$$
\hat{\psi}_{1}^{+}\left(\mathbf{u}_{1}\right) \hat{\psi}_{1}\left(\mathbf{v}_{1}\right) \rightarrow \hat{\psi}_{1}^{+} \widetilde{\left(\mathbf{u}_{1}\right) \hat{\psi}_{1}}\left(\mathbf{v}_{1}\right)=\tilde{\hat{\psi}}_{1}^{+}\left(\mathbf{u}_{1}\right) \tilde{\hat{\psi}}_{1}\left(\mathbf{v}_{1}\right)
$$

Similarly, one gets

$$
\begin{aligned}
& \hat{\psi}_{1}\left(\mathbf{u}_{1}\right) \hat{\psi}_{1}\left(\mathbf{v}_{1}\right) \rightarrow \hat{\psi}_{1}\left(\widetilde{\left.\mathbf{u}_{1}\right) \hat{\psi}_{1}}\left(\mathbf{v}_{1}\right)=\tilde{\hat{\psi}}_{1}\left(\mathbf{u}_{1}\right) \tilde{\hat{\psi}}_{1}\left(\mathbf{v}_{1}\right),\right. \\
& \hat{\psi}_{1}^{+}\left(\mathbf{u}_{1}\right) \hat{\psi}_{2}\left(\mathbf{v}_{1}\right) \rightarrow \hat{\psi}_{1}^{+}\left(\widetilde{\left.\mathbf{u}_{1}\right) \hat{\psi}_{2}}\left(\mathbf{v}_{1}\right)=\tilde{\hat{\psi}}_{1}^{+}\left(\mathbf{u}_{1}\right) \tilde{\hat{\psi}}_{2}\left(\mathbf{v}_{1}\right)\right.
\end{aligned}
$$


In general case the following formula is valid:

$$
\hat{\psi}_{i_{1}}^{+}\left(\mathbf{u}_{i_{1}}\right) \ldots \hat{\psi}_{i_{n}}^{+}\left(\mathbf{u}_{i_{n}}\right) \hat{\psi}_{j_{1}}\left(\mathbf{v}_{j_{1}}\right) \ldots \hat{\psi}_{j_{m}}\left(\mathbf{v}_{j_{m}}\right) \rightarrow \hat{\psi}_{i_{1}}^{+}\left(\mathbf{u}_{i_{1}}\right) \ldots \hat{\psi}_{j_{m}}\left(\mathbf{v}_{j_{m}}\right)=\tilde{\hat{\psi}}_{i_{1}}^{+}\left(\mathbf{u}_{1}\right) \ldots \tilde{\hat{\psi}}_{j_{m}}\left(\mathbf{v}_{j_{m}}\right)
$$

To explain this formula we note that each of $\hat{\psi}_{j}\left(\mathbf{v}_{j}\right)$ (or $\hat{\psi}_{i}^{+}\left(\mathbf{u}_{i}\right)$ ) entering $\hat{A}(\mathbf{u}, \mathbf{v})$ (see (13)) is related to other operators $\hat{\psi}^{+}$(or $\hat{\psi}$ ) not entering $\hat{A}(\mathbf{u}, \mathbf{v})$ only by unique way, which leads to the binary relations (19), (20). Therefore, we come to (21) by sorting out all the operators $\hat{\psi}_{i}, \hat{\psi}_{i^{\prime}}$ containing in $\hat{A}(\mathbf{u}, \mathbf{v})$.

The operators $\hat{\psi}_{i}(\mathbf{x}), \hat{\psi}_{j}\left(\mathbf{x}^{\prime}\right)$ are anticommutative. For this reason there is a question concerning the consistency of (20), (21). The anticommutativity of $\tilde{\hat{\psi}}_{i}(\mathbf{x}), \tilde{\hat{\psi}}_{j}\left(\mathbf{x}^{\prime}\right)$ (and also $\tilde{\hat{\psi}}_{i}^{+}(\mathbf{x}), \tilde{\hat{\psi}}_{j}^{+}\left(\mathbf{x}^{\prime}\right)$ ) represents the consistency condition of these formulas,

$$
\left\{\tilde{\hat{\psi}}_{i}(\mathbf{x}), \tilde{\hat{\psi}}_{j}\left(\mathbf{x}^{\prime}\right)\right\}=\left\{\tilde{\hat{\psi}}_{i}^{+}(\mathbf{x}), \tilde{\hat{\psi}}_{j}^{+}\left(\mathbf{x}^{\prime}\right)\right\}=0 .
$$

The validity of these anticommutative relations can be easily proved if to use the definitions (16)-(18) for $\tilde{\hat{\psi}}, \tilde{\hat{\psi}}^{+}$and the commutative relations for $\hat{\chi}, \hat{\chi}^{+}$and $\hat{\eta}_{\alpha}, \hat{\eta}_{\alpha}^{+}$.

\section{THE OPERATORS OF BASIC PHYSICAL QUANTITIES}

In this section we consider the operators of basic physical quantities, which act in the Hilbert space $\tilde{H}$. Let us start from the density operator for particles of the first kind. The corresponding operator acting in the original Hilbert space $H$ is of the form

$$
\hat{\rho}_{1}(\mathbf{x})=\hat{\psi}_{1}^{+}(\mathbf{x}) \hat{\psi}_{1}(\mathbf{x})
$$

Hence, in accordance with (20), one finds

$$
\tilde{\hat{\rho}}_{1}(\mathbf{x})=\tilde{\hat{\psi}}_{1}^{+}(\mathbf{x}) \tilde{\hat{\psi}}_{1}(\mathbf{x})=\hat{\chi}_{1}^{+}(\mathbf{x}) \hat{\chi}_{1}(\mathbf{x})+\hat{O}_{1}^{+}(\mathbf{x}) \hat{\chi}_{1}(\mathbf{x})+\hat{\chi}_{1}^{+}(\mathbf{x}) \hat{O}_{1}(\mathbf{x})+\hat{O}_{1}^{+}(\mathbf{x}) \hat{O}_{1}(\mathbf{x})
$$

Note that the operators with zero matrix elements in the subspace $\tilde{H}_{a}$ appear in the right-hand side of (19) because of points $\mathbf{u}_{1}$ and $\mathbf{v}_{1}$ are close to each other. Since

$$
\hat{O}_{1}^{+}(\mathbf{x}) \hat{\chi}_{1}(\mathbf{x})=\int d \mathbf{y} \hat{\varphi}^{+}(\mathbf{x}, \mathbf{y}) \hat{\chi}_{2}(\mathbf{y}) \hat{\chi}_{1}(\mathbf{x}), \quad \hat{\chi}_{1}^{+}(\mathbf{x}) \hat{O}_{1}(\mathbf{x})=\int d \mathbf{y} \hat{\chi}_{1}^{+}(\mathbf{x}) \hat{\chi}_{2}^{+}(\mathbf{y}) \hat{\varphi}(\mathbf{x}, \mathbf{y})
$$

and $\hat{\varphi}(\mathbf{x}, \mathbf{y})$ differs from zero only for $|\mathbf{x}-\mathbf{y}| \lesssim a$, these operators according to (12) do not have the matrix elements in the subspace $\tilde{H}_{a}$ and therefore can be omitted. Using the permutation relation $\left\{\hat{\chi}_{2}^{+}\left(\mathbf{z}_{1}\right), \hat{\chi}_{2}\left(\mathbf{z}_{2}\right)\right\}=\delta\left(\mathbf{z}_{1}-\mathbf{z}_{2}\right)$ the operator $\hat{O}_{1}^{+}(\mathbf{x}) \hat{O}_{1}(\mathbf{x})$ is written as

$$
\hat{O}_{1}^{+}(\mathbf{x}) \hat{O}_{1}(\mathbf{x})=\int d \mathbf{z} \hat{\varphi}^{+}(\mathbf{x}, \mathbf{z}) \hat{\varphi}(\mathbf{x}, \mathbf{z})+\int d \mathbf{z}_{1} d \mathbf{z}_{2} \hat{\varphi}^{+}\left(\mathbf{x}, \mathbf{z}_{2}\right) \hat{\varphi}\left(\mathbf{x}, \mathbf{z}_{1}\right) \hat{\chi}_{2}^{+}\left(\mathbf{z}_{1}\right) \hat{\chi}_{2}\left(\mathbf{z}_{2}\right) .
$$

The matrix element of the second term is zero in the subspace $\tilde{H}_{a}$ because of $\mathbf{z}_{1} \approx \mathbf{z}_{2} \approx \mathbf{x}$ (see (12)). For this reason this term can be omitted. Thus, with the use of the method that was described in previous section we have

$$
\tilde{\hat{\rho}}_{1}(\mathbf{x})=\hat{\chi}_{1}^{+}(\mathbf{x}) \hat{\chi}_{1}(\mathbf{x})+\int d \mathbf{z} \hat{\varphi}^{+}(\mathbf{x}, \mathbf{z}) \hat{\varphi}(\mathbf{x}, \mathbf{z})
$$


Similarly, if $\hat{\rho}_{2}(\mathbf{x})=\hat{\psi}_{2}^{+}(\mathbf{x}) \hat{\psi}_{2}(\mathbf{x})$ represents the density operator for particles of the second kind, then $\hat{\rho}_{2}(\mathbf{x}) \rightarrow \tilde{\hat{\rho}}_{2}(\mathbf{x})$, where

$$
\tilde{\hat{\rho}}_{2}(\mathbf{x})=\hat{\chi}_{2}^{+}(\mathbf{x}) \hat{\chi}_{2}(\mathbf{x})+\int d \mathbf{z} \hat{\varphi}^{+}(\mathbf{z}, \mathbf{x}) \hat{\varphi}(\mathbf{z}, \mathbf{x})
$$

Bearing in mind (16) and the assumption concerning the "small radius" of the bound state, one gets the following formulas:

$$
\int d \mathbf{z} \hat{\varphi}^{+}(\mathbf{x}, \mathbf{z}) \hat{\varphi}(\mathbf{x}, \mathbf{z}) \approx \hat{\eta}_{\alpha}^{+}(\mathbf{x}) \hat{\eta}_{\alpha}(\mathbf{x}), \quad \int d \mathbf{z} \hat{\varphi}^{+}(\mathbf{z}, \mathbf{x}) \hat{\varphi}(\mathbf{z}, \mathbf{x}) \approx \hat{\eta}_{\alpha}^{+}(\mathbf{x}) \hat{\eta}_{\alpha}(\mathbf{x})
$$

which allow us to obtain the densities operators for particles of the first and second kinds,

$$
\tilde{\hat{\rho}}_{1}(\mathbf{x})=\hat{\chi}_{1}^{+}(\mathbf{x}) \hat{\chi}_{1}(\mathbf{x})+\hat{\eta}_{\alpha}^{+}(\mathbf{x}) \hat{\eta}_{\alpha}(\mathbf{x}), \quad \tilde{\hat{\rho}}_{2}(\mathbf{x})=\hat{\chi}_{2}^{+}(\mathbf{x}) \hat{\chi}_{2}(\mathbf{x})+\hat{\eta}_{\alpha}^{+}(\mathbf{x}) \hat{\eta}_{\alpha}(\mathbf{x})
$$

Thus, the operators $\hat{\eta}_{\alpha}^{+}(\mathbf{x}), \hat{\eta}_{\alpha}(\mathbf{x})$ can be interpreted as the creation and annihilation operators of the bound states with quantum numbers $\alpha$ at the point $\mathbf{x}$, and $\hat{\eta}_{\alpha}^{+}(\mathbf{x}) \hat{\eta}_{\alpha}(\mathbf{x})$ as the density operator of the bound states. For example, the first formula from (24) have a simple physical meaning: the density of particles of the first kind is equal to the sum of densities for free particles of the same kind and bound states (each bound state contains one particle of the first kind).

Consider a state vector $\Phi(\mathbf{X})=\hat{\eta}_{\alpha}^{+}(\mathbf{X})|0\rangle$, which specifies a compound particle at the point $\mathbf{X}$ (this state vector corresponds to continuous spectrum). Then, in accordance with (24), we have

$$
\left(\Phi(\mathbf{X}), \hat{\rho}_{1}\left(\mathbf{x}_{1}\right) \Phi\left(\mathbf{X}^{\prime}\right)\right)=\delta\left(\mathbf{X}-\mathbf{X}^{\prime}\right)\left(\frac{M}{m_{2}}\right)^{3}\left|\varphi_{\alpha}\left(\frac{M}{m_{2}}\left(\mathbf{x}_{1}-\mathbf{X}\right)\right)\right|^{2} .
$$

For a wave packet

$$
\Psi_{\mathbf{X}_{0}}=\int d \mathbf{X} f_{\mathbf{X}_{0}}(\mathbf{X}) \Phi(\mathbf{X}), \quad \int d \mathbf{X}\left|f_{\mathbf{X}_{0}}(\mathbf{X})\right|^{2}=1
$$

the quantity

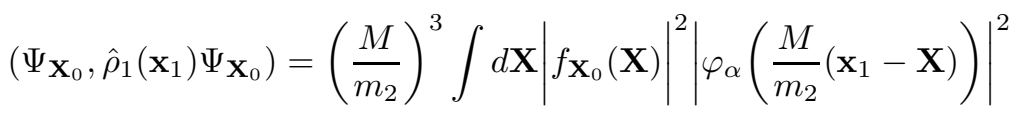

should be treated as the probability density to find the first particle at the point $\mathbf{x}_{1}$ if the atom is in a state $\Psi_{\mathbf{X}_{0}}$. If the bound state is localized near a point $\mathbf{X}_{0}$ (i.e., $\left|f_{\mathbf{X}_{0}}(\mathbf{X})\right|^{2} \rightarrow \delta\left(\mathbf{X}-\mathbf{X}_{0}\right)$ ), then

$$
\left(\Psi_{\mathbf{X}_{0}}, \hat{\rho}_{1}\left(\mathbf{x}_{1}\right) \Psi_{\mathbf{X}_{0}}\right) \rightarrow\left(\frac{M}{m_{2}}\right)^{3}\left|\varphi_{\alpha}\left(\frac{M}{m_{2}}\left(\mathbf{x}_{1}-\mathbf{X}_{0}\right)\right)\right|^{2}
$$

Since $\left(M / m_{2}\right)\left(\mathbf{x}_{1}-\mathbf{X}_{0}\right)=\mathbf{x}=\mathbf{x}_{1}-\mathbf{x}_{2}$, we come (as it should be) to the probability distribution for the space coordinate of the first particle in atom (the atom is at the point $\mathbf{X}_{0}$ ).

Let us find now the momentum density operator in the space $\tilde{H}$. In original Hilbert space, the momentum density operator $\hat{\boldsymbol{\pi}}_{1}(\mathbf{x})$ for particles of the first kind is defined as

$$
\hat{\boldsymbol{\pi}}_{1}(\mathbf{x})=-\frac{i}{2}\left(\hat{\psi}_{1}^{+}(\mathbf{x}) \frac{\partial \hat{\psi}_{1}(\mathbf{x})}{\partial \mathbf{x}}-\frac{\partial \hat{\psi}_{1}^{+}(\mathbf{x})}{\partial \mathbf{x}} \hat{\psi}_{1}(\mathbf{x})\right) .
$$


Then according to (20)

$$
\hat{\boldsymbol{\pi}}_{1}(\mathbf{x}) \rightarrow \tilde{\boldsymbol{\pi}}_{1}(\mathbf{x})=-\frac{i}{2}\left(\tilde{\hat{\psi}}_{1}^{+}(\mathbf{x}) \frac{\partial \tilde{\hat{\psi}}_{1}(\mathbf{x})}{\partial \mathbf{x}}-\frac{\partial \tilde{\hat{\psi}}_{1}^{+}(\mathbf{x})}{\partial \mathbf{x}} \tilde{\hat{\psi}}_{1}(\mathbf{x})\right) .
$$

Following the derivation of (22), (23) for $\tilde{\hat{\rho}}_{1}(\mathbf{x})$ and $\tilde{\hat{\rho}}_{2}(\mathbf{x})$ we obtain

$$
\begin{aligned}
& \tilde{\hat{\boldsymbol{\pi}}}_{1}(\mathbf{x})=-\frac{i}{2}\left(\hat{\chi}_{1}^{+}(\mathbf{x}) \frac{\partial \hat{\chi}_{1}(\mathbf{x})}{\partial \mathbf{x}}-\frac{\partial \hat{\chi}_{1}^{+}(\mathbf{x})}{\partial \mathbf{x}} \hat{\chi}_{1}(\mathbf{x})\right)-\frac{i}{2} \int d \mathbf{y}\left(\hat{\varphi}^{+}(\mathbf{x}, \mathbf{y}) \frac{\partial \hat{\varphi}(\mathbf{x}, \mathbf{y})}{\partial \mathbf{x}}-\frac{\partial \hat{\varphi}^{+}(\mathbf{x}, \mathbf{y})}{\partial \mathbf{x}} \hat{\varphi}(\mathbf{x}, \mathbf{y})\right), \\
& \tilde{\hat{\boldsymbol{\pi}}}_{2}(\mathbf{x})=-\frac{i}{2}\left(\hat{\chi}_{2}^{+}(\mathbf{x}) \frac{\partial \hat{\chi}_{2}(\mathbf{x})}{\partial \mathbf{x}}-\frac{\partial \hat{\chi}_{2}^{+}(\mathbf{x})}{\partial \mathbf{x}} \hat{\chi}_{2}(\mathbf{x})\right)-\frac{i}{2} \int d \mathbf{y}\left(\hat{\varphi}^{+}(\mathbf{y}, \mathbf{x}) \frac{\partial \hat{\varphi}(\mathbf{y}, \mathbf{x})}{\partial \mathbf{x}}-\frac{\partial \hat{\varphi}^{+}(\mathbf{y}, \mathbf{x})}{\partial \mathbf{x}} \hat{\varphi}(\mathbf{y}, \mathbf{x})\right) .
\end{aligned}
$$

It is convenient for our further consideration to rewrite (25), (26) in terms of the center of mass variables $\mathbf{y}=\mathbf{y}_{1}-\mathbf{y}_{2}$ and $\mathbf{Y}=\left(m_{1} \mathbf{y}_{1}+m_{2} \mathbf{y}_{2}\right) /\left(m_{1}+m_{2}\right)$ :

$$
\begin{aligned}
\tilde{\boldsymbol{\pi}}_{1}(\mathbf{x}) & =-\frac{i}{2}\left(\hat{\chi}_{1}^{+}(\mathbf{x}) \frac{\partial \hat{\chi}_{1}(\mathbf{x})}{\partial \mathbf{x}}-\frac{\partial \hat{\chi}_{1}^{+}(\mathbf{x})}{\partial \mathbf{x}} \hat{\chi}_{1}(\mathbf{x})\right)-\frac{i}{2} \int d \mathbf{y} d \mathbf{Y} \delta\left(\mathbf{x}-\mathbf{Y}-\frac{m_{2}}{M} \mathbf{y}\right)\left\{\hat{\varphi}^{+}(\mathbf{y}, \mathbf{Y}) \frac{\partial \hat{\varphi}(\mathbf{y}, \mathbf{Y})}{\partial \mathbf{y}}\right. \\
& \left.-\frac{\partial \hat{\varphi}^{+}(\mathbf{y}, \mathbf{Y})}{\partial \mathbf{y}} \hat{\varphi}(\mathbf{y}, \mathbf{Y})+\frac{m_{1}}{M}\left(\hat{\varphi}^{+}(\mathbf{y}, \mathbf{Y}) \frac{\partial \hat{\varphi}(\mathbf{y}, \mathbf{Y})}{\partial \mathbf{Y}}-\frac{\partial \hat{\varphi}^{+}(\mathbf{y}, \mathbf{Y})}{\partial \mathbf{Y}} \hat{\varphi}(\mathbf{y}, \mathbf{Y})\right)\right\}, \\
\tilde{\hat{\pi}}_{2}(\mathbf{x}) & =-\frac{i}{2}\left(\hat{\chi}_{2}^{+}(\mathbf{x}) \frac{\partial \hat{\chi}_{2}(\mathbf{x})}{\partial \mathbf{x}}-\frac{\partial \hat{\chi}_{2}^{+}(\mathbf{x})}{\partial \mathbf{x}} \hat{\chi}_{2}(\mathbf{x})\right)-\frac{i}{2} \int d \mathbf{y} d \mathbf{Y} \delta\left(\mathbf{x}-\mathbf{Y}+\frac{m_{1}}{M} \mathbf{y}\right)\left\{-\hat{\varphi}^{+}(\mathbf{y}, \mathbf{Y}) \frac{\partial \hat{\varphi}(\mathbf{y}, \mathbf{Y})}{\partial \mathbf{y}}\right. \\
& \left.+\frac{\partial \hat{\varphi}^{+}(\mathbf{y}, \mathbf{Y})}{\partial \mathbf{y}} \hat{\varphi}(\mathbf{y}, \mathbf{Y})+\frac{m_{2}}{M}\left(\hat{\varphi}^{+}(\mathbf{y}, \mathbf{Y}) \frac{\partial \hat{\varphi}(\mathbf{y}, \mathbf{Y})}{\partial \mathbf{Y}}-\frac{\partial \hat{\varphi}^{+}(\mathbf{y}, \mathbf{Y})}{\partial \mathbf{Y}} \hat{\varphi}(\mathbf{y}, \mathbf{Y})\right)\right\},
\end{aligned}
$$

where $\hat{\varphi}\left(\mathbf{y}_{1}, \mathbf{y}_{2}\right) \equiv \hat{\varphi}(\mathbf{y}, \mathbf{Y})$. Note that in terms of these variables the operators $\tilde{\hat{\rho}}_{1}(\mathbf{x})$ and $\tilde{\hat{\rho}}_{2}(\mathbf{x})$ have the form

$$
\begin{aligned}
& \tilde{\hat{\rho}}_{1}(\mathbf{x})=\hat{\chi}_{1}^{+}(\mathbf{x}) \hat{\chi}_{1}(\mathbf{x})+\int d \mathbf{y} d \mathbf{Y} \delta\left(\mathbf{x}-\mathbf{Y}-\frac{m_{2}}{M} \mathbf{y}\right) \hat{\varphi}^{+}(\mathbf{y}, \mathbf{Y}) \hat{\varphi}(\mathbf{y}, \mathbf{Y}), \\
& \tilde{\hat{\rho}}_{2}(\mathbf{x})=\hat{\chi}_{2}^{+}(\mathbf{x}) \hat{\chi}_{2}(\mathbf{x})+\int d \mathbf{y} d \mathbf{Y} \delta\left(\mathbf{x}-\mathbf{Y}+\frac{m_{1}}{M} \mathbf{y}\right) \hat{\varphi}^{+}(\mathbf{y}, \mathbf{Y}) \hat{\varphi}(\mathbf{y}, \mathbf{Y}) .
\end{aligned}
$$

It is clear that (27), (28) can be expressed through the creation and annihilation operators $\hat{\eta}_{\alpha}^{+}(\mathbf{x}), \hat{\eta}_{\alpha}(\mathbf{x})$ of atoms if to employ (17). Taking into account (27) it is easy to find the operator $\tilde{\hat{\boldsymbol{\pi}}}=\tilde{\hat{\boldsymbol{\pi}}}_{1}(\mathbf{x})+\tilde{\hat{\boldsymbol{\pi}}}_{2}(\mathbf{x})$ of the total momentum density of the system in the approximation, where the radius of bound state is small,

$\tilde{\hat{\boldsymbol{\pi}}}=-\frac{i}{2}\left(\hat{\chi}_{1}^{+}(\mathbf{x}) \frac{\partial \hat{\chi}_{1}(\mathbf{x})}{\partial \mathbf{x}}-\frac{\partial \hat{\chi}_{1}^{+}(\mathbf{x})}{\partial \mathbf{x}} \hat{\chi}_{1}(\mathbf{x})\right)-\frac{i}{2}\left(\hat{\chi}_{2}^{+}(\mathbf{x}) \frac{\partial \hat{\chi}_{2}(\mathbf{x})}{\partial \mathbf{x}}-\frac{\partial \hat{\chi}_{2}^{+}(\mathbf{x})}{\partial \mathbf{x}} \hat{\chi}_{2}(\mathbf{x})\right)-\frac{i}{2}\left(\hat{\eta}_{\alpha}^{+}(\mathbf{x}) \frac{\partial \hat{\eta}_{\alpha}(\mathbf{x})}{\partial \mathbf{x}}-\frac{\partial \hat{\eta}_{\alpha}^{+}(\mathbf{x})}{\partial \mathbf{x}} \hat{\eta}_{\alpha}(\mathbf{x})\right)$.

The third term in this formula is in accordance with interpretation of $\hat{\eta}_{\alpha}^{+}(\mathbf{x}), \hat{\eta}_{\alpha}(\mathbf{x})$ as the creation and annihilation operators of the bound state with quantum numbers $\alpha$ at the point $\mathbf{x}$.

Finally let us consider a Hamiltonian in the space $\tilde{H}$. We suppose that this Hamiltonian has a standard form in the Hilbert space $H$ and can be written as

$$
\hat{\mathcal{H}}=\hat{\mathcal{H}}_{0}+\hat{V}
$$

where $\hat{\mathcal{H}}_{0}$ and $\hat{V}$ are the operators of kinetic energy and potential energy given by

$$
\begin{gathered}
\hat{\mathcal{H}}_{0}=\sum_{i=1}^{2} \frac{1}{2 m_{i}} \int d \mathbf{x} \frac{\partial \hat{\psi}_{i}^{+}(\mathbf{x})}{\partial \mathbf{x}} \frac{\partial \hat{\psi}_{i}(\mathbf{x})}{\partial \mathbf{x}} \\
\hat{V}=\frac{1}{2} \sum_{i, j=1}^{2} \int d \mathbf{x} d \mathbf{x}^{\prime} \hat{\psi}_{i}^{+}(\mathbf{x}) \hat{\psi}_{j}^{+}\left(\mathbf{x}^{\prime}\right) \nu_{i j}\left(\mathbf{x}-\mathbf{x}^{\prime}\right) \hat{\psi}_{j}\left(\mathbf{x}^{\prime}\right) \hat{\psi}_{i}(\mathbf{x}),
\end{gathered}
$$


and $\nu_{i j}\left(\mathbf{x}-\mathbf{x}^{\prime}\right)$ is a potential energy of interaction for particles of the kinds $i$ and $j$. After the similar calculations that led us to the expressions for $\tilde{\hat{\rho}}, \tilde{\hat{\pi}}$, we obtain

$$
\hat{\mathcal{H}}_{0} \rightarrow \tilde{\hat{\mathcal{H}}}_{0}=\sum_{i=1}^{2} \frac{1}{2 m_{i}}\left(\int d \mathbf{x} \frac{\partial \hat{\chi}_{i}^{+}(\mathbf{x})}{\partial \mathbf{x}} \frac{\partial \hat{\chi}_{i}(\mathbf{x})}{\partial \mathbf{x}}+\int d \mathbf{x}_{1} d \mathbf{x}_{2} \frac{\partial \hat{\varphi}^{+}\left(\mathbf{x}_{1}, \mathbf{x}_{2}\right)}{\partial \mathbf{x}_{i}} \frac{\partial \hat{\varphi}\left(\mathbf{x}_{1}, \mathbf{x}_{2}\right)}{\partial \mathbf{x}_{i}}\right) .
$$

Next changing to the center of mass variables (see (17)) and noting that

$$
\frac{\partial}{\partial \mathbf{x}_{1}}=\frac{\partial}{\partial \mathbf{x}}+\frac{m_{1}}{M} \frac{\partial}{\partial \mathbf{X}}, \quad \frac{\partial}{\partial \mathbf{x}_{2}}=-\frac{\partial}{\partial \mathbf{x}}+\frac{m_{2}}{M} \frac{\partial}{\partial \mathbf{X}}
$$

one gets

$$
\tilde{\hat{\mathcal{H}}}_{0}=\sum_{i=1}^{2} \frac{1}{2 m_{i}} \int d \mathbf{x} \frac{\partial \hat{\chi}_{i}^{+}(\mathbf{x})}{\partial \mathbf{x}} \frac{\partial \hat{\chi}_{i}(\mathbf{x})}{\partial \mathbf{x}}-\frac{1}{2 \mu} \int d \mathbf{x} d \mathbf{X} \hat{\eta}_{\alpha}^{+}(\mathbf{X}) \hat{\eta}_{\beta}(\mathbf{X}) \frac{\partial \varphi_{\alpha}^{*}(\mathbf{x})}{\partial \mathbf{x}} \frac{\partial \varphi_{\beta}(\mathbf{x})}{\partial \mathbf{x}}+\frac{1}{2 M} \int d \mathbf{X} \frac{\partial \hat{\eta}_{\alpha}^{+}(\mathbf{X})}{\partial \mathbf{X}} \frac{\partial \hat{\eta}_{\alpha}(\mathbf{X})}{\partial \mathbf{X}}
$$

where $\mu=m_{1} m_{2} /\left(m_{1}+m_{2}\right)$ is a reduced mass.

Let us find now $\tilde{\hat{V}}(\hat{V} \rightarrow \tilde{\hat{V}})$. According to (20) we have

$$
\tilde{\hat{V}}=\frac{1}{2} \sum_{i, j=1}^{2} \int d \mathbf{x} d \mathbf{x}^{\prime} \tilde{\hat{\psi}}_{i}^{+}(\mathbf{x}) \tilde{\hat{\psi}}_{j}^{+}\left(\mathbf{x}^{\prime}\right) \nu_{i j}\left(\mathbf{x}-\mathbf{x}^{\prime}\right) \tilde{\hat{\psi}}_{j}\left(\mathbf{x}^{\prime}\right) \tilde{\hat{\psi}}_{i}(\mathbf{x}),
$$

where $\tilde{\hat{\psi}}_{i}(\mathbf{x})=\hat{\chi}_{i}(\mathbf{x})+\hat{O}_{i}(\mathbf{x})$ (see (201) $)$. Thus, $\tilde{\hat{V}}$ can be represented in the form

$$
\tilde{\hat{V}}=\tilde{\hat{V}}_{0}+\tilde{\hat{V}}_{1}+\tilde{\hat{V}}_{2}+\tilde{\hat{V}}_{3}+\tilde{\hat{V}}_{4}
$$

where $\tilde{\hat{V}}_{k}(k=0, \ldots 4)$ contains $k$ multipliers of type $\hat{\chi}$ and $4-k$ multipliers of type $\hat{O}$. The operators $\hat{O}_{i}(\mathbf{x})$ have, according to (16), (18), the form

$$
\begin{gathered}
\hat{O}_{i}(\mathbf{x})=\int d \mathbf{y} \hat{\varphi}_{i}(\mathbf{x}, \mathbf{y}) \hat{\chi}_{i^{\prime}}^{+}(\mathbf{y}), \\
\hat{\varphi}_{1}(\mathbf{x}, \mathbf{y})=\hat{\varphi}(\mathbf{x}, \mathbf{y}), \quad \hat{\varphi}_{2}(\mathbf{x}, \mathbf{y})=\hat{\varphi}(\mathbf{y}, \mathbf{x}),
\end{gathered}
$$

where index $i^{\prime}$ is defined as $1^{\prime}=2,2^{\prime}=1$. Then $\tilde{\hat{V}}_{0}$ is of the form

$$
\begin{aligned}
\tilde{\hat{V}}_{0}=\frac{1}{2} \sum_{i, j=1}^{2} \int d \mathbf{x}_{1} d \mathbf{x}_{2} \nu_{i j}\left(\mathbf{x}_{1}-\mathbf{x}_{2}\right) \\
\quad \times \int d \mathbf{y}_{1} d \mathbf{y}_{2} d \mathbf{y}_{3} d \mathbf{y}_{4} \hat{\varphi}_{i}^{+}\left(\mathbf{x}_{1}, \mathbf{y}_{1}\right) \hat{\varphi}_{j}^{+}\left(\mathbf{x}_{2}, \mathbf{y}_{2}\right) \hat{\varphi}_{j}\left(\mathbf{x}_{2}, \mathbf{y}_{3}\right) \hat{\varphi}_{i}\left(\mathbf{x}_{1}, \mathbf{y}_{4}\right) \hat{\chi}_{i^{\prime}}\left(\mathbf{y}_{1}\right) \hat{\chi}_{j^{\prime}}\left(\mathbf{y}_{2}\right) \hat{\chi}_{j^{\prime}}^{+}\left(\mathbf{y}_{3}\right) \hat{\chi}_{i^{\prime}}^{+}\left(\mathbf{y}_{4}\right) .
\end{aligned}
$$

Note that the operators $\hat{\varphi}$ and $\hat{\varphi}^{+}$in (33) are normally ordered, whereas $\hat{\chi}$ and $\hat{\chi}^{+}$are not normally ordered. Therefore, we put them in order using Wick's theorem:

$$
\begin{aligned}
& \hat{\chi}_{i^{\prime}}\left(\mathbf{y}_{1}\right) \hat{\chi}_{j^{\prime}}\left(\mathbf{y}_{2}\right) \hat{\chi}_{j^{\prime}}^{+}\left(\mathbf{y}_{3}\right) \hat{\chi}_{i^{\prime}}^{+}\left(\mathbf{y}_{4}\right)=: \hat{\chi}_{i^{\prime}}\left(\mathbf{y}_{1}\right) \hat{\chi}_{j^{\prime}}\left(\mathbf{y}_{2}\right) \hat{\chi}_{j^{\prime}}^{+}\left(\mathbf{y}_{3}\right) \hat{\chi}_{i^{\prime}}^{+}\left(\mathbf{y}_{4}\right):+\underset{a}{\hat{\chi}_{i^{\prime}}}\left(\mathbf{y}_{1}\right) \hat{\chi}_{j^{\prime}}\left(\mathbf{y}_{2}\right) \hat{\chi}_{j^{\prime}}^{+}\left(\mathbf{y}_{3}\right) \hat{\chi}_{i^{\prime}}^{+}\left(\mathbf{y}_{4}\right): \\
& +: \underset{a}{\hat{\chi}_{i^{\prime}}}\left(\mathbf{y}_{1}\right) \hat{\chi}_{j^{\prime}}\left(\mathbf{y}_{2}\right) \hat{\chi}_{j^{\prime}}^{+}\left(\mathbf{y}_{3}\right) \underset{a}{\hat{\chi}_{i^{\prime}}}\left(\mathbf{y}_{4}\right):+: \hat{\chi}_{i^{\prime}}\left(\mathbf{y}_{1}\right) \underset{a}{\hat{\chi}_{j^{\prime}}} \underset{a}{\left(\mathbf{y}_{2}\right)} \underset{\hat{\chi}_{j^{\prime}}}{+}\left(\mathbf{y}_{3}\right) \hat{\chi}_{i^{\prime}}^{+}\left(\mathbf{y}_{4}\right):+: \hat{\chi}_{i^{\prime}}\left(\mathbf{y}_{1}\right) \hat{\chi}_{j^{\prime}}\left(\mathbf{y}_{2}\right) \hat{\chi}_{j^{\prime}}^{+}\left(\mathbf{y}_{3}\right) \hat{\chi}_{i^{\prime}}^{+}\left(\mathbf{y}_{4}\right):
\end{aligned}
$$

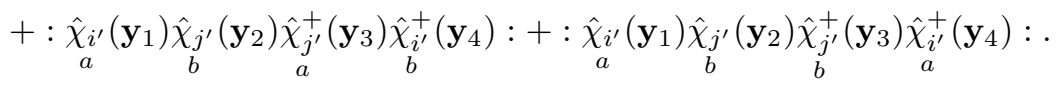


The operator $\hat{\varphi}(\mathbf{x}, \mathbf{y}) \equiv \varphi_{\alpha}(\mathbf{x}-\mathbf{y}) \hat{\eta}_{\alpha}\left(\frac{m_{1} \mathbf{x}+m_{2} \mathbf{y}}{m_{1}+m_{2}}\right)$ differs from zero only for $\mathbf{x} \approx \mathbf{y},(|\mathbf{x}-\mathbf{y}|<a)$. Thus, only those of $\varphi_{i}$, for which $\left|\mathbf{y}_{1}-\mathbf{y}_{4}\right|<a$ contribute to the integral over $\mathbf{y}$ in (33). This means that the first term in (34) in virtue of (12) does not contribute to the matrix element of $\tilde{\hat{V}}_{0}$ taken between the states belonging to $\tilde{H}_{a}$ because of $\hat{\varphi}_{i}\left(\mathbf{x}_{1}, \mathbf{y}_{4}\right) \hat{\chi}_{i}\left(\mathbf{y}_{1}\right) \Phi=0$. Similarly, one can prove that the terms, which contain the single contractions in (34) do not give a contribution to the matrix element of $\tilde{\hat{V}}_{0}$ taken between the states in $\tilde{H}_{a}$. The penultimate term in (34) containing the double contractions does not also contribute to the above mentioned matrix element. Indeed, the penultimate term in (34) equals to $\delta\left(\mathbf{y}_{1}-\mathbf{y}_{3}\right) \delta\left(\mathbf{y}_{2}-\mathbf{y}_{4}\right)$. In this case the nonzero matrix element exists for $\mathbf{x}_{1} \approx \mathbf{x}_{2}$ and $\hat{\varphi}_{j}\left(\mathbf{x}_{2}, \mathbf{y}_{3}\right) \hat{\varphi}_{i}\left(\mathbf{x}_{1}, \mathbf{y}_{4}\right) \Phi=0$ in virtue of (12). Thus, only the latter term in (34) equal $\delta\left(\mathbf{y}_{2}-\mathbf{y}_{3}\right) \delta\left(\mathbf{y}_{1}-\mathbf{y}_{4}\right)$ can give the contribution to the matrix element of $\tilde{\hat{V}}_{0}$. Therefore, not changing the matrix elements in $\tilde{H}_{a}$, the operator $\tilde{\hat{V}}_{0}$ can be represented in the form

$$
\tilde{\hat{V}}_{0}=\frac{1}{2} \sum_{i, j=1}^{2} \int d \mathbf{x}_{1} d \mathbf{x}_{2} d \mathbf{y}_{1} d \mathbf{y}_{2} \hat{\varphi}_{i}^{+}\left(\mathbf{x}_{1}, \mathbf{y}_{1}\right) \hat{\varphi}_{j}^{+}\left(\mathbf{x}_{2}, \mathbf{y}_{2}\right) \nu_{i j}\left(\mathbf{x}_{1}-\mathbf{x}_{2}\right) \hat{\varphi}_{j}\left(\mathbf{x}_{2}, \mathbf{y}_{2}\right) \hat{\varphi}_{i}\left(\mathbf{x}_{1}, \mathbf{y}_{1}\right),
$$

or, according to (32),

$$
\begin{aligned}
\tilde{\hat{V}}_{0}=\frac{1}{2} \int d \mathbf{x}_{1} d \mathbf{x}_{2} d \mathbf{y}_{1} d \mathbf{y}_{2} \hat{\varphi}^{+} & \left(\mathbf{x}_{1}, \mathbf{y}_{1}\right) \hat{\varphi}^{+}\left(\mathbf{x}_{2}, \mathbf{y}_{2}\right) \\
& \times\left\{\nu_{11}\left(\mathbf{x}_{1}-\mathbf{x}_{2}\right)+\nu_{22}\left(\mathbf{y}_{1}-\mathbf{y}_{2}\right)+\nu_{12}\left(\mathbf{x}_{1}-\mathbf{y}_{2}\right)+\nu_{21}\left(\mathbf{y}_{1}-\mathbf{x}_{2}\right)\right\} \hat{\varphi}\left(\mathbf{x}_{2}, \mathbf{y}_{2}\right) \hat{\varphi}\left(\mathbf{x}_{1}, \mathbf{y}_{1}\right) .
\end{aligned}
$$

Similarly, noting that

$$
\begin{aligned}
& \tilde{\hat{V}}_{1}=\frac{1}{2} \sum_{i, j=1}^{2} \int d \mathbf{x}_{1} d \mathbf{x}_{2} d \mathbf{y} \nu_{i j}\left(\mathbf{x}_{1}-\mathbf{x}_{2}\right) \\
& \times\left\{\hat{\varphi}_{i}^{+}\left(\mathbf{x}_{1}, \mathbf{y}\right) \hat{\chi}_{i^{\prime}}(\mathbf{y}) \hat{\chi}_{j}^{+}\left(\mathbf{x}_{2}\right) \hat{\chi}_{j}\left(\mathbf{x}_{2}\right) \hat{\chi}_{i}\left(\mathbf{x}_{1}\right)+\hat{\varphi}_{j}^{+}\left(\mathbf{x}_{2}, \mathbf{y}\right) \hat{\chi}_{i}\left(\mathbf{x}_{1}\right) \hat{\chi}_{j^{\prime}}^{+}(\mathbf{y}) \hat{\chi}_{j}\left(\mathbf{x}_{2}\right) \hat{\chi}_{i}\left(\mathbf{x}_{1}\right)+h . c .\right\} \\
& \tilde{\hat{V}}_{3}=\frac{1}{2} \sum_{i, j=1}^{2} \int d \mathbf{x}_{1} d \mathbf{x}_{2} d \mathbf{y}_{1} d \mathbf{y}_{2} d \mathbf{y}_{3} \nu_{i j}\left(\mathbf{x}_{1}-\mathbf{x}_{2}\right)\left\{\hat{\varphi}_{i}^{+}\left(\mathbf{x}_{1}, \mathbf{y}_{1}\right) \hat{\varphi}_{j}^{+}\left(\mathbf{x}_{2}, \mathbf{y}_{2}\right) \hat{\varphi}_{j}\left(\mathbf{x}_{2}, \mathbf{y}_{3}\right) \hat{\chi}_{i^{\prime}}\left(\mathbf{y}_{1}\right) \hat{\chi}_{j^{\prime}}\left(\mathbf{y}_{2}\right) \hat{\chi}_{j^{\prime}}^{+}\left(\mathbf{y}_{3}\right) \hat{\chi}_{i}\left(\mathbf{x}_{1}\right)\right. \\
& \left.+\hat{\varphi}_{i}^{+}\left(\mathbf{x}_{1}, \mathbf{y}_{1}\right) \hat{\varphi}_{j}^{+}\left(\mathbf{x}_{2}, \mathbf{y}_{2}\right) \hat{\varphi}_{i}\left(\mathbf{x}_{1}, \mathbf{y}_{3}\right) \hat{\chi}_{i^{\prime}}\left(\mathbf{y}_{1}\right) \hat{\chi}_{j^{\prime}}\left(\mathbf{y}_{2}\right) \hat{\chi}_{j}\left(\mathbf{x}_{2}\right) \hat{\chi}_{i^{\prime}}^{+}\left(\mathbf{y}_{3}\right)+h . c .\right\}
\end{aligned}
$$

and performing the same derivation as for $\tilde{\hat{V}}_{0}$, it is easy to verify (using the anticommutative relations for $\hat{\chi}, \hat{\chi}^{+}$and (34)) that we can consider $\tilde{\hat{V}}_{1}=\tilde{\hat{V}}_{3}=0$ not changing the matrix elements of $\tilde{\hat{V}}_{1}$ and $\tilde{\hat{V}}_{3}$ in the subspace $\tilde{H}_{a}$. Next it is evident that the following formula is valid:

$$
\tilde{\hat{V}}_{4}=\frac{1}{2} \sum_{i, j=1}^{2} \int d \mathbf{x}_{1} d \mathbf{x}_{2} \hat{\chi}_{i}^{+}\left(\mathbf{x}_{1}\right) \hat{\chi}_{j}^{+}\left(\mathbf{x}_{2}\right) \nu_{i j}\left(\mathbf{x}_{1}-\mathbf{x}_{2}\right) \hat{\chi}_{j}\left(\mathbf{x}_{2}\right) \hat{\chi}_{i}\left(\mathbf{x}_{1}\right) .
$$

Finally let us find $\tilde{\hat{V}}_{2}$,

$$
\begin{aligned}
\tilde{\hat{V}}_{2}=\frac{1}{2} \sum_{i, j=1}^{2} \int d \mathbf{x}_{1} d \mathbf{x}_{2} \nu_{i j}\left(\mathbf{x}_{1}-\mathbf{x}_{2}\right)\left\{\hat{O}_{i}^{+}\left(\mathbf{x}_{1}\right) \hat{O}_{j}^{+}\left(\mathbf{x}_{2}\right) \hat{\chi}_{j}\left(\mathbf{x}_{2}\right) \hat{\chi}_{i}\left(\mathbf{x}_{1}\right)+\hat{O}_{i}^{+}\left(\mathbf{x}_{1}\right) \hat{\chi}_{j}^{+}\left(\mathbf{x}_{2}\right) \hat{O}_{j}\left(\mathbf{x}_{2}\right) \hat{\chi}_{i}\left(\mathbf{x}_{1}\right)+h . c .\right. \\
\left.+\hat{O}_{i}^{+}\left(\mathbf{x}_{1}\right) \hat{\chi}_{j}^{+}\left(\mathbf{x}_{2}\right) \hat{\chi}_{j}\left(\mathbf{x}_{2}\right) \hat{O}_{i}\left(\mathbf{x}_{1}\right)+\hat{\chi}_{i}^{+}\left(\mathbf{x}_{1}\right) \hat{O}_{j}^{+}\left(\mathbf{x}_{2}\right) \hat{O}_{j}\left(\mathbf{x}_{2}\right) \hat{\chi}_{i}\left(\mathbf{x}_{1}\right)\right\} .
\end{aligned}
$$


It can be easily seen that the first two terms and the corresponding Hermitian conjugate terms do not give a contribution to the matrix element of $\tilde{\hat{V}}_{2}$ in the subspace $\tilde{H}_{a}$. Therefore, we can consider

$$
\begin{aligned}
\tilde{\hat{V}}_{2}=\frac{1}{2} \sum_{i, j=1}^{2} \int d \mathbf{x}_{1} d \mathbf{x}_{2} d \mathbf{y}_{1} d \mathbf{y}_{2} \nu_{i j}\left(\mathbf{x}_{1}-\mathbf{x}_{2}\right)\left\{\hat{\varphi}_{i}^{+}\left(\mathbf{x}_{1}, \mathbf{y}_{1}\right) \hat{\varphi}_{i}\left(\mathbf{x}_{1}, \mathbf{y}_{2}\right) \hat{\chi}_{i^{\prime}}\left(\mathbf{y}_{1}\right) \hat{\chi}_{j}^{+}\left(\mathbf{x}_{2}\right) \hat{\chi}_{j}\left(\mathbf{x}_{2}\right) \hat{\chi}_{i^{\prime}}^{+}\left(\mathbf{y}_{2}\right)\right. \\
\left.+\hat{\varphi}_{j}^{+}\left(\mathbf{x}_{2}, \mathbf{y}_{1}\right) \hat{\varphi}_{j}\left(\mathbf{x}_{2}, \mathbf{y}_{2}\right) \hat{\chi}_{i}^{+}\left(\mathbf{x}_{1}\right) \hat{\chi}_{j^{\prime}}\left(\mathbf{y}_{1}\right) \hat{\chi}_{j^{\prime}}^{+}\left(\mathbf{y}_{2}\right) \hat{\chi}_{i}\left(\mathbf{x}_{1}\right)\right\} .
\end{aligned}
$$

The first and second terms in this expression contribute to the matrix element of $\tilde{\hat{V}}_{2}$ in $\tilde{H}_{a}$ under the following arrangements of contractions:

$$
\begin{aligned}
& : \hat{\chi}_{i^{\prime}}\left(\mathbf{y}_{1}\right) \underset{a}{\hat{\chi}_{j}^{+}}\left(\mathbf{x}_{2}\right) \underset{b}{\hat{\chi}_{j}}\left(\mathbf{x}_{2}\right) \hat{\chi}_{i^{\prime}}^{+}\left(\mathbf{y}_{2}\right):+\underset{a}{\hat{\chi}_{i^{\prime}}}\left(\mathbf{y}_{1}\right) \hat{\chi}_{j}^{+}\left(\mathbf{x}_{2}\right) \hat{\chi}_{j}\left(\mathbf{x}_{2}\right) \underset{a}{\hat{\chi}_{i^{\prime}}^{+}}\left(\mathbf{y}_{2}\right):=\delta_{j i^{\prime}} \delta\left(\mathbf{y}_{1}-\mathbf{x}_{2}\right) \delta\left(\mathbf{y}_{2}-\mathbf{x}_{2}\right)+\delta\left(\mathbf{y}_{1}-\mathbf{y}_{2}\right) \hat{\chi}_{j}^{+}\left(\mathbf{x}_{2}\right) \hat{\chi}_{j}\left(\mathbf{x}_{2}\right), \\
& : \hat{\chi}_{i}^{+}\left(\mathbf{x}_{1}\right) \hat{\chi}_{a}^{j^{\prime}}\left(\mathbf{y}_{1}\right) \hat{\chi}_{j^{\prime}}^{+}\left(\mathbf{y}_{2}\right) \hat{\chi}_{i}\left(\mathbf{x}_{1}\right):=\delta\left(\mathbf{y}_{1}-\mathbf{y}_{2}\right) \hat{\chi}_{i}^{+}\left(\mathbf{x}_{1}\right) \hat{\chi}_{i}\left(\mathbf{x}_{1}\right)
\end{aligned}
$$

Thus, we have

$$
\begin{gathered}
\tilde{\hat{V}}_{2}=\int d \mathbf{x}_{1} d \mathbf{x}_{2} \nu_{12}\left(\mathbf{x}_{1}-\mathbf{x}_{2}\right) \hat{\varphi}^{+}\left(\mathbf{x}_{1}, \mathbf{x}_{2}\right) \hat{\varphi}\left(\mathbf{x}_{1}, \mathbf{x}_{2}\right)+\int d \mathbf{x}_{1} d \mathbf{x}_{2} \hat{\varphi}^{+}\left(\mathbf{x}_{2}, \mathbf{y}_{2}\right) \hat{\varphi}\left(\mathbf{x}_{2}, \mathbf{y}_{2}\right) \\
\times\left\{\nu_{11}\left(\mathbf{x}_{1}-\mathbf{x}_{2}\right) \hat{\chi}_{1}^{+}\left(\mathbf{x}_{1}\right) \hat{\chi}_{1}\left(\mathbf{x}_{1}\right)+\nu_{21}\left(\mathbf{x}_{1}-\mathbf{y}_{2}\right) \hat{\chi}_{1}^{+}\left(\mathbf{x}_{1}\right) \hat{\chi}_{1}\left(\mathbf{x}_{1}\right)+\nu_{22}\left(\mathbf{x}_{1}-\mathbf{y}_{2}\right) \hat{\chi}_{2}^{+}\left(\mathbf{x}_{1}\right) \hat{\chi}_{2}\left(\mathbf{x}_{1}\right)+\nu_{12}\left(\mathbf{x}_{1}-\mathbf{x}_{2}\right) \hat{\chi}_{2}^{+}\left(\mathbf{x}_{1}\right) \hat{\chi}_{2}\left(\mathbf{x}_{1}\right)\right\}
\end{gathered}
$$

The first term in this formula quadratic in field operators can be combined with the latter term in (31). As a result we obtain

$$
\begin{aligned}
\int d \mathbf{x}_{1} d \mathbf{x}_{2} \nu_{12}\left(\mathbf{x}_{1}-\mathbf{x}_{2}\right) \hat{\varphi}^{+}\left(\mathbf{x}_{1}, \mathbf{x}_{2}\right) \hat{\varphi}\left(\mathbf{x}_{1}, \mathbf{x}_{2}\right)-\frac{1}{2 \mu} \int d \mathbf{x} d \mathbf{X} \hat{\eta}_{\alpha}^{+}(\mathbf{X}) \hat{\eta}_{\alpha}(\mathbf{X}) \frac{\partial \varphi_{\alpha}^{*}(\mathbf{x})}{\partial \mathbf{x}} \frac{\partial \varphi_{\alpha}(\mathbf{x})}{\partial \mathbf{x}}= \\
\int d \mathbf{x} d \mathbf{X} \hat{\eta}_{\alpha}^{+}(\mathbf{X}) \hat{\eta}_{\beta}(\mathbf{X}) \varphi_{\alpha}^{*}(\mathbf{x})\left\{-\frac{1}{2 \mu} \Delta_{\mathbf{x}}+\nu_{12}(\mathbf{x})\right\} \varphi_{\beta}(\mathbf{x}) .
\end{aligned}
$$

Since $\varphi_{\beta}(\mathbf{x})$ satisfies the Schroedinger equation

$$
\left\{-\frac{1}{2 \mu} \Delta_{\mathbf{x}}+\nu_{12}(\mathbf{x})\right\} \varphi_{\beta}(\mathbf{x})=\varepsilon_{\beta} \varphi_{\beta}(\mathbf{x})
$$

where $\varepsilon_{\beta}$ are the atomic energy levels, the latter formula takes the form

$$
\int d \mathbf{X} \sum_{\alpha} \varepsilon_{\alpha} \hat{\eta}_{\alpha}^{+}(\mathbf{X}) \hat{\eta}_{\alpha}(\mathbf{X})
$$

Hence, taking into account (30), (31), (36), the Hamiltonian of the system $\tilde{\hat{\mathcal{H}}}$ is of the form

$$
\tilde{\hat{\mathcal{H}}}=\tilde{\hat{\mathcal{H}}}_{0}+\tilde{\hat{\mathcal{H}}}_{i n t}^{1}+\tilde{\hat{\mathcal{H}}}_{i n t}^{2}+\tilde{\hat{\mathcal{H}}}_{\text {int }}^{3}
$$

where

$$
\tilde{\hat{\mathcal{H}}}_{0}=\sum_{j=1}^{2} \frac{1}{2 m_{j}} \int d \mathbf{x} \frac{\partial \hat{\chi}_{j}^{+}(\mathbf{x})}{\partial \mathbf{x}} \frac{\partial \hat{\chi}_{j}(\mathbf{x})}{\partial \mathbf{x}}+\sum_{\alpha} \int d \mathbf{X}\left\{\frac{1}{2 M} \frac{\partial \hat{\eta}_{\alpha}^{+}(\mathbf{X})}{\partial \mathbf{X}} \frac{\partial \hat{\eta}_{\alpha}(\mathbf{X})}{\partial \mathbf{X}}+\varepsilon_{\alpha} \hat{\eta}_{\alpha}^{+}(\mathbf{X}) \hat{\eta}_{\alpha}(\mathbf{X})\right\}
$$

is the Hamiltonian for free particles and bound states, and 


$$
\begin{aligned}
& \tilde{\hat{\mathcal{H}}}_{\text {int }}^{1}=\int d \mathbf{x}_{1} d \mathbf{x}_{2} d \mathbf{y}_{2} \hat{\varphi}^{+}\left(\mathbf{x}_{2}, \mathbf{y}_{2}\right) \hat{\varphi}\left(\mathbf{x}_{2}, \mathbf{y}_{2}\right)\left\{\left(\nu_{11}\left(\mathbf{x}_{1}-\mathbf{x}_{2}\right)+\nu_{21}\left(\mathbf{x}_{1}-\mathbf{y}_{2}\right)\right) \hat{\chi}_{1}^{+}\left(\mathbf{x}_{1}\right) \hat{\chi}_{1}\left(\mathbf{x}_{1}\right)\right. \\
& \left.+\left(\nu_{22}\left(\mathbf{x}_{1}-\mathbf{y}_{2}\right)+\nu_{12}\left(\mathbf{x}_{1}-\mathbf{x}_{2}\right)\right) \hat{\chi}_{2}^{+}\left(\mathbf{x}_{1}\right) \hat{\chi}_{2}\left(\mathbf{x}_{1}\right)\right\}, \\
& \tilde{\hat{\mathcal{H}}}_{i n t}^{2}=\frac{1}{2} \int d \mathbf{x}_{1} d \mathbf{x}_{2} d \mathbf{y}_{1} d \mathbf{y}_{2} \hat{\varphi}^{+}\left(\mathbf{x}_{1}, \mathbf{y}_{1}\right) \hat{\varphi}^{+}\left(\mathbf{x}_{2}, \mathbf{y}_{2}\right) \hat{\varphi}\left(\mathbf{x}_{2}, \mathbf{y}_{2}\right) \hat{\varphi}\left(\mathbf{x}_{1}, \mathbf{y}_{1}\right) \\
& \times\left\{\nu_{11}\left(\mathbf{x}_{1}-\mathbf{x}_{2}\right)+\nu_{22}\left(\mathbf{y}_{1}-\mathbf{y}_{2}\right)+\nu_{12}\left(\mathbf{x}_{1}-\mathbf{y}_{2}\right)+\nu_{21}\left(\mathbf{y}_{1}-\mathbf{x}_{2}\right)\right\}, \\
& \tilde{\hat{\mathcal{H}}}_{\text {int }}^{3}=\frac{1}{2} \int d \mathbf{x}_{1} d \mathbf{x}_{2}\left\{\nu_{11}\left(\mathbf{x}_{1}-\mathbf{x}_{2}\right) \hat{\chi}_{1}^{+}\left(\mathbf{x}_{1}\right) \hat{\chi}_{1}^{+}\left(\mathbf{x}_{2}\right) \hat{\chi}_{1}\left(\mathbf{x}_{2}\right) \hat{\chi}_{1}\left(\mathbf{x}_{1}\right)\right. \\
& \left.+\nu_{22}\left(\mathbf{x}_{1}-\mathbf{x}_{2}\right) \hat{\chi}_{2}^{+}\left(\mathbf{x}_{1}\right) \hat{\chi}_{2}^{+}\left(\mathbf{x}_{2}\right) \hat{\chi}_{2}\left(\mathbf{x}_{2}\right) \hat{\chi}_{2}\left(\mathbf{x}_{1}\right)+2 \nu_{12}\left(\mathbf{x}_{1}-\mathbf{x}_{2}\right) \hat{\chi}_{1}^{+}\left(\mathbf{x}_{1}\right) \hat{\chi}_{1}\left(\mathbf{x}_{1}\right) \hat{\chi}_{2}^{+}\left(\mathbf{x}_{2}\right) \hat{\chi}_{2}\left(\mathbf{x}_{2}\right)\right\}
\end{aligned}
$$

are the Hamiltonians of interaction. The Hamiltonian $\tilde{\hat{\mathcal{H}}}_{\text {int }}^{1}$ corresponds to scattering of particles of the first and

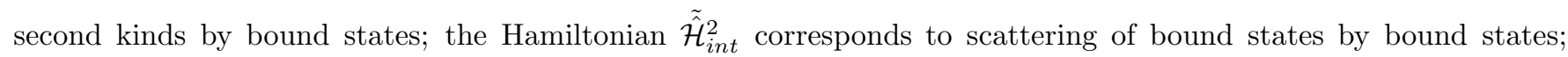
finally, the Hamiltonian $\tilde{\hat{\mathcal{H}}}_{\text {int }}^{3}$ corresponds to scattering of particles of the first and second kinds by particles of the same kinds. The Hamiltonians of interaction (38), (39) may be written through the creation $\hat{\eta}_{\alpha}^{+}(\mathbf{x})$ and annihilation $\hat{\eta}_{\alpha}(\mathbf{x})$ operators of atoms by using of (17). We want to emphasize that the obtained Hamiltonians of interaction do not lead to decay processes and formation of compound particles as it should be in the low-energy approximation. This fact reflects that atoms are absolutely stable in the main approximation.

In conclusion of this section we address to the Galilean invariance of the developed theory. We suppose that the initial theory based only on the field operators $\hat{\psi}_{i}(\mathbf{x}), \hat{\psi}_{i}^{+}(\mathbf{x}),(i=1,2)$ is Galilean invariant. This means that under the following transformations:

$$
\hat{\psi}_{i}(\mathbf{x}) \rightarrow \hat{\psi}_{i}^{\prime}(\mathbf{x})=e^{i m_{i} \mathbf{v} \mathbf{x}} \hat{\psi}_{i}(\mathbf{x})
$$

the operators for particles number $\hat{N}_{i}$, momentum $\hat{\mathcal{P}}_{k}$ and energy $\hat{\mathcal{H}}$ transform as follows:

$$
\begin{gathered}
\hat{N}_{i} \rightarrow \hat{N}_{i}^{\prime}=\hat{N}_{i}, \quad i=1,2, \\
\hat{\mathcal{P}}_{k} \rightarrow \hat{\mathcal{P}}_{k}^{\prime}=\hat{\mathcal{P}}_{k}+v_{k}\left(\hat{N}_{1}+\hat{N}_{2}\right), \quad \hat{\mathcal{H}} \rightarrow \hat{\mathcal{H}}^{\prime}=\hat{\mathcal{H}}+v_{k} \hat{\mathcal{P}}_{k}+\frac{1}{2} m_{1} v^{2} \hat{N}_{1}+\frac{1}{2} m_{2} \hat{N}_{2} v^{2} .
\end{gathered}
$$

Since $\hat{\psi}_{i}(\mathbf{x})$ and $\hat{\psi}_{i}^{\prime}(\mathbf{x})$ meet the same commutation relations, they are related to each other by the unitary transformation

$$
\hat{\psi}_{i}^{\prime}(\mathbf{x})=U_{v} \hat{\psi}_{i}(\mathbf{x}) U_{v}^{+}
$$

where the unitary operator $U_{v}$, is defined by ${ }^{8}$ :

$$
U_{v}=\exp \left(-i \mathbf{v} m_{1} \int d \mathbf{x} \mathbf{x} \hat{\rho}_{1}(\mathbf{x})-i \mathbf{v} m_{2} \int d \mathbf{x} \mathbf{x} \hat{\rho}_{2}(\mathbf{x})\right) .
$$


Let us show that

$$
\tilde{U}_{v}=\exp \left(-i \mathbf{v} m_{1} \int d \mathbf{x} \mathbf{x} \tilde{\hat{\rho}}_{1}(\mathbf{x})-i \mathbf{v} m_{2} \int d \mathbf{x} \mathbf{x} \tilde{\hat{\rho}}_{2}(\mathbf{x})\right)
$$

defines the Galilei transformations in the presence of bound states of particles (compare to (41)). To this end we note that this operator can be written, according to (28), in the form

$$
\tilde{U}_{v}=\exp \left(-i \mathbf{v} \sum_{i=1}^{2} m_{i} \int d \mathbf{x} \hat{\chi}_{i}^{+}(\mathbf{x}) \hat{\chi}_{i}(\mathbf{x})-i \mathbf{v} \hat{\mathbf{s}}\right)
$$

where

$$
\begin{aligned}
\hat{\mathbf{s}}=m_{1} \int d \mathbf{x}_{1} d \mathbf{x}_{2} \mathbf{x}_{1} \hat{\varphi}^{+}\left(\mathbf{x}_{1}, \mathbf{x}_{2}\right) \hat{\varphi}\left(\mathbf{x}_{1}, \mathbf{x}_{2}\right) & +m_{2} \int d \mathbf{x}_{1} d \mathbf{x}_{2} \mathbf{x}_{1} \hat{\varphi}^{+}\left(\mathbf{x}_{2}, \mathbf{x}_{1}\right) \hat{\varphi}\left(\mathbf{x}_{2}, \mathbf{x}_{1}\right) \\
& =\int d \mathbf{x}_{1} d \mathbf{x}_{2}\left(m_{1} \mathbf{x}_{1}+m_{2} \mathbf{x}_{2}\right) \hat{\varphi}^{+}\left(\mathbf{x}_{1}, \mathbf{x}_{2}\right) \hat{\varphi}\left(\mathbf{x}_{1}, \mathbf{x}_{2}\right) .
\end{aligned}
$$

Since

$$
\hat{\varphi}\left(\mathbf{x}_{1}, \mathbf{x}_{2}\right)=\varphi_{\alpha}(\mathbf{x}) \hat{\eta}_{\alpha}(\mathbf{X}), \quad \int d \mathbf{x} \varphi_{\alpha}^{*}(\mathbf{x}) \varphi_{\beta}(\mathbf{x})=\delta_{\alpha \beta},
$$

changing to the center of mass variables one finds,

$$
\hat{\mathbf{s}}=\left(m_{1}+m_{2}\right) \int d \mathbf{X X} \sum_{\alpha} \hat{\eta}_{\alpha}^{+}(\mathbf{X}) \hat{\eta}_{\alpha}(\mathbf{X})
$$

Therefore, just as we expected

$$
\tilde{U}_{v}=\exp \left(-i \mathbf{v} \sum_{i=1}^{2} m_{i} \int d \mathbf{x} \hat{\chi}_{i}^{+}(\mathbf{x}) \hat{\chi}_{i}(\mathbf{x})-i \mathbf{v}\left(m_{1}+m_{2}\right) \int d \mathbf{X X} \sum_{\alpha} \hat{\eta}_{\alpha}^{+}(\mathbf{X}) \hat{\eta}_{\alpha}(\mathbf{X})\right) .
$$

The employment of this formula and the canonic permutation relations result in

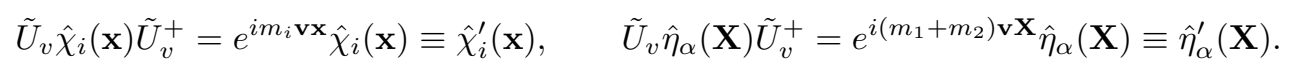

Since the quantity $\hat{\varphi}^{+}(\mathbf{x}, \mathbf{X}) \hat{\varphi}(\mathbf{x}, \mathbf{X})$ is not changed under such transformation, the Hamiltonians of interaction $\tilde{\hat{\mathcal{H}}}_{\text {int }}^{1}$,

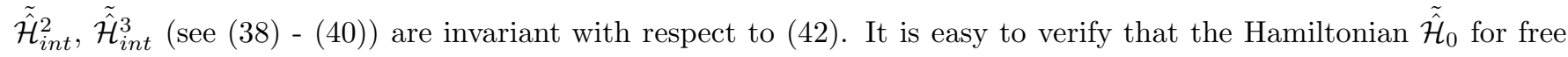
particles and bound states transforms according to the law

$$
\tilde{U}_{v} \tilde{\hat{\mathcal{H}}}_{0} \tilde{U}_{v}^{+}=\tilde{\hat{\mathcal{H}}}_{0}+v_{k} \tilde{\hat{\mathcal{P}}}_{k}+\frac{m_{1} v^{2}}{2} \tilde{\hat{\hat{N}}}_{1}+\frac{m_{2} v^{2}}{2} \tilde{\hat{\hat{N}}}_{2}+\frac{\left(m_{1}+m_{2}\right) v^{2}}{2} \tilde{\hat{N}}_{b}
$$

Hence,

$$
\begin{gathered}
\tilde{U}_{v} \tilde{\hat{\mathcal{H}}} \tilde{U}_{v}^{+}=\tilde{\hat{\mathcal{H}}}+v_{k} \tilde{\hat{\mathcal{P}}}_{k}+\frac{m_{1} v^{2}}{2} \tilde{\hat{N}}_{1}+\frac{m_{2} v^{2}}{2} \tilde{\hat{N}}_{2}+\frac{\left(m_{1}+m_{2}\right) v^{2}}{2} \tilde{\hat{N}}_{b} \\
\tilde{U}_{v} \tilde{\hat{\mathcal{P}}}_{k} \tilde{U}_{v}^{+}=\tilde{\hat{\mathcal{P}}}_{k}+m_{1} v_{k} \tilde{\hat{N}}_{1}+m_{2} v_{k} \tilde{\hat{N}}_{2}+\left(m_{1}+m_{2}\right) v_{k} \tilde{\hat{N}}_{b}
\end{gathered}
$$

where $\tilde{\hat{\mathcal{P}}}_{k}, \tilde{\hat{N}}_{1}, \tilde{\hat{N}}_{2}, \tilde{\hat{N}}_{b}$ are the momentum operator and the particle number operators (for free particles of the first and second kinds and their bound states). These formulas prove the Galilean invariance of the developed theory. Finally we note the validity of the following formula:

$$
\tilde{U}_{v} \tilde{\hat{\boldsymbol{\pi}}}_{i}(\mathbf{x}) \tilde{U}_{v}^{+}=\tilde{\hat{\boldsymbol{\pi}}}_{i}(\mathbf{x})+m_{i} \mathbf{v} \tilde{\hat{\rho}}_{i}(\mathbf{x}),
$$

which follows from (27), (28) by using (42). 


\section{ELECTROMAGNETIC INTERACTION}

Here we consider the electromagnetic interaction assuming that the formation of bound states of particles is caused by Coulomb's (electromagnetic) forces. Therefore, the potential energy $\nu_{i j}\left(\mathbf{x}-\mathbf{x}^{\prime}\right)$ entering (38)-(40) should be written as

$$
\nu_{i j}\left(\mathbf{x}-\mathbf{x}^{\prime}\right)=\frac{e_{i} e_{j}}{\left|\mathbf{x}-\mathbf{x}^{\prime}\right|}, \quad i, j=1,2 .
$$

We also introduce the additional interactions of particles (see below) with an external electromagnetic field $\mathbf{A}^{(e)}(\mathbf{x}, t)$, $\varphi^{(e)}(\mathbf{x}, t)$ and quantized electromagnetic field specified by a potential $\hat{\mathbf{a}}(\mathbf{x})$ (Coulomb's gauge)

$$
\hat{\mathbf{A}}(\mathbf{x}, t)=\hat{\mathbf{a}}(\mathbf{x})+\mathbf{A}^{(e)}(\mathbf{x}, t),
$$

where $^{8}$

$$
\hat{\mathbf{a}}(\mathbf{x})=\sum_{\mathbf{k}} \sum_{\lambda=1}^{2}\left(\frac{2 \pi}{\mathcal{V} \omega_{k}}\right)^{1 / 2}\left(\mathbf{e}_{\mathbf{k} \lambda} \hat{C}_{\mathbf{k} \lambda} e^{i \mathbf{k x}}+\text { h.c. }\right)
$$

$\left(\hat{C}_{\mathbf{k} \lambda}\right.$ is the annihilation operator of a photon with momentum $k$ and polarization $\left.\mathbf{e}_{\mathbf{k} \lambda}\right)$. As in section II, we identify the subspace $\tilde{H}_{a}$ to $\tilde{H}$ assuming that the matrix elements of the operators in coordinate representation give the main contribution to the quantum electrodynamics processes and correspond to the space scale $\Delta x \gtrsim a\left(a \rightarrow 0, a>>r_{0}\right)$.

The density $\varepsilon(\mathbf{x})$ of a Hamiltonian in the second quantization representation has the well-known form

$$
\hat{\varepsilon}(\mathbf{x})=\hat{\varepsilon}_{f}(\mathbf{x})+\hat{\varepsilon}_{C}(\mathbf{x})+\sum_{i=1}^{2} e_{i} \hat{\psi}_{i}^{+}(\mathbf{x}) \hat{\psi}_{i}(\mathbf{x}) \varphi^{(e)}(\mathbf{x}, t)+\sum_{i=1}^{2} \frac{1}{2 m_{i}}\left(\frac{\partial}{\partial \mathbf{x}}-i e_{i} \hat{\mathbf{A}}(\mathbf{x}, t)\right) \hat{\psi}_{i}^{+}(\mathbf{x})\left(\frac{\partial}{\partial \mathbf{x}}+i e_{i} \hat{\mathbf{A}}(\mathbf{x}, t)\right) \hat{\psi}_{i}(\mathbf{x}),
$$

where $\hat{\varepsilon}_{f}(\mathbf{x})$ is the energy density of a free electromagnetic field, $\hat{\varepsilon}_{C}(\mathbf{x})$ is the energy density of Coulomb's interaction, the third term describes the interaction between the particles and external scalar potential. This formula leads to the following Hamiltonian of the system:

$$
\hat{\mathcal{H}}(t)=\hat{\mathcal{H}}_{0}+\hat{\mathcal{H}}_{i n t}+\hat{V}(t), \quad \hat{\mathcal{H}}_{0}=\hat{\mathcal{H}}_{f}+\hat{\mathcal{H}}_{p}
$$

where

$$
\begin{gathered}
\hat{\mathcal{H}}_{f} \equiv \int d \mathbf{x} \hat{\varepsilon}_{f}(\mathbf{x})=\sum_{\mathbf{k}, \lambda} \omega_{k} \hat{C}_{\mathbf{k} \lambda}^{+} \hat{C}_{\mathbf{k} \lambda}, \\
\hat{\mathcal{H}}_{p}=\sum_{i=1}^{2} \frac{1}{2 m_{i}} \int d \mathbf{x} \frac{\partial \hat{\psi}_{i}^{+}(\mathbf{x})}{\partial \mathbf{x}} \frac{\partial \hat{\psi}_{i}(\mathbf{x})}{\partial \mathbf{x}}, \\
\hat{\mathcal{H}}_{i n t}=\int d \mathbf{x} \hat{\varepsilon}_{C}(\mathbf{x})
\end{gathered}
$$

are the Hamiltonians for free photons, free particles, and Coulomb's interaction respectively. The operator $\hat{V}(t)$ represents a Hamiltonian that describes the interaction of particles with the electromagnetic fields $\hat{\mathbf{A}}(\mathbf{x}, t), \varphi^{(e)}(\mathbf{x}, t)$

$$
\hat{V}(t)=-\int d \mathbf{x} \hat{\mathbf{A}}(\mathbf{x}, t) \hat{\mathbf{J}}(\mathbf{x}, t)-\frac{1}{2} \int d \mathbf{x} \hat{\mathbf{A}}^{2}(\mathbf{x}, t) \sum_{i=1}^{2} \frac{e_{i}}{m_{i}} \hat{\sigma}_{i}(\mathbf{x})+\int d \mathbf{x} \varphi^{(e)}(\mathbf{x}, t) \hat{\sigma},
$$




$$
\hat{\mathbf{J}}(\mathbf{x}, t)=-\hat{\mathbf{A}}(\mathbf{x}, t) \sum_{i=1}^{2} \frac{e_{i}}{m_{i}} \hat{\sigma}_{i}(\mathbf{x})+\hat{\mathbf{j}}_{0}(\mathbf{x}), \quad \hat{\mathbf{j}}_{0}(\mathbf{x})=\sum_{i=1}^{2} \frac{e_{i}}{m_{i}} \hat{\boldsymbol{\pi}}_{i}(\mathbf{x}),
$$

where $\hat{\sigma}_{i}(x)=e_{i} \hat{\rho}_{i}(x)$ is the charge density for particles of the kind $i, \hat{\sigma}=\hat{\sigma}_{1}+\hat{\sigma}_{2}$. This Hamiltonian is exact and describes (in this sense) the processes in which the bound states of particles are involved. Since $\hat{\mathbf{a}}(\mathbf{x})$ commute among themselves and with $\hat{\psi}_{i}(\mathbf{x}), \hat{\psi}_{i}^{+}(\mathbf{x})$, in order to find the effective Hamiltonian $\tilde{\hat{\mathcal{H}}}(t)$ that describes electrodynamic processes at low-energies in the presence of bound states of particles we need, in accordance with section IV, to replace the operators $\hat{\psi}_{i}, \hat{\psi}_{i}^{+}$, â by $\tilde{\hat{\psi}}_{i}, \tilde{\hat{\psi}}_{i}^{+}, \tilde{\hat{\mathbf{a}}}$ :

$$
\tilde{\hat{\mathcal{H}}}(t)=\left.\hat{\mathcal{H}}(t)\right|_{\hat{\psi}_{1} \rightarrow \tilde{\hat{\psi}}_{1}, \hat{\psi}_{2} \rightarrow \tilde{\hat{\psi}}_{2}, \hat{\mathbf{a}} \rightarrow \tilde{\mathbf{a}}},
$$

where

$$
\tilde{\hat{\psi}}_{1}(\mathbf{x})=\hat{\chi}_{1}(\mathbf{x})+\int d \mathbf{y} \hat{\varphi}(\mathbf{x}, \mathbf{y}) \hat{\chi}_{2}^{+}(\mathbf{y}), \quad \tilde{\hat{\psi}}_{2}(\mathbf{x})=\hat{\chi}_{2}(\mathbf{x})+\int d \mathbf{y} \hat{\varphi}(\mathbf{y}, \mathbf{x}) \hat{\chi}_{1}^{+}(\mathbf{y}), \quad \tilde{\hat{\mathbf{a}}}=\hat{\mathbf{a}}
$$

$(\tilde{\hat{\mathbf{a}}}(\mathbf{x})$ coincides with $\hat{\mathbf{a}}(\mathbf{x})$ and acts in the space $\tilde{H})$. As a result the effective Hamiltonian is defined by (44)-(49), in which

$$
\hat{\sigma}_{i}(\mathbf{x}) \rightarrow \tilde{\hat{\sigma}}_{i}(\mathbf{x})=e_{i} \tilde{\hat{\rho}}_{i}(\mathbf{x}), \quad \hat{\boldsymbol{\pi}}_{i}(\mathbf{x}) \rightarrow \tilde{\hat{\boldsymbol{\pi}}}_{i}(\mathbf{x}), \quad \hat{\psi}_{i}(\mathbf{x}) \rightarrow \tilde{\hat{\psi}}_{i}(\mathbf{x})
$$

moreover, the operators $\tilde{\hat{\rho}}_{i}(\mathbf{x}), \tilde{\boldsymbol{\pi}}_{i}(\mathbf{x}), \tilde{\hat{\psi}}_{i}(\mathbf{x})$ containing the creation $\hat{\eta}_{\alpha}^{+}$and annihilation $\hat{\eta}_{\alpha}$ operators of bound states are determined by (27), (28), (16)-(18).

Let us define the operator of magnetic field

$$
\hat{\mathbf{H}}(\mathbf{x}, t)=\hat{\mathbf{h}}(\mathbf{x})+\hat{\mathbf{H}}^{(e)}(\mathbf{x}, t)
$$

where

$$
\hat{\mathbf{h}}(\mathbf{x})=\operatorname{rot} \hat{\mathbf{a}}(\mathbf{x}), \quad \hat{\mathbf{H}}^{(e)}(\mathbf{x}, t)=\operatorname{rot} \mathbf{A}^{(e)}(\mathbf{x}, t)
$$

and the operator of electric field

$$
\hat{\mathbf{E}}(\mathbf{x}, t)=\hat{\mathbf{e}}(\mathbf{x})+\hat{\mathbf{E}}^{(e)}(\mathbf{x}, t)
$$

where

$$
\hat{\mathbf{e}}(\mathbf{x})=\hat{\mathbf{e}}^{t}(\mathbf{x})+\hat{\mathbf{e}}^{l}(\mathbf{x}), \quad \hat{\mathbf{E}}^{(e)}(\mathbf{x}, t)=-\frac{\partial}{\partial t} \mathbf{A}^{(e)}(\mathbf{x}, t)-\frac{\partial}{\partial \mathbf{x}} \varphi^{(e)}(\mathbf{x}, t)
$$

The transverse $\hat{\mathbf{e}}^{t}(\mathbf{x})$ and longitudinal $\hat{\mathbf{e}}^{l}(\mathbf{x})$ components of the electric field created by particles are defined as follows:

$$
\hat{\mathbf{e}}^{l}(\mathbf{x})=-\frac{\partial}{\partial \mathbf{x}} \hat{\mathbf{a}}_{0}(\mathbf{x}), \quad \hat{\mathbf{e}}^{t}(\mathbf{x})=-\dot{\hat{\mathbf{a}}}(\mathbf{x}) \equiv-i[\tilde{\hat{\mathcal{H}}}(t), \hat{\mathbf{a}}(\mathbf{x})]
$$


Here $\hat{\mathbf{a}}_{0}(\mathbf{x})$ represents the operator of the scalar potential,

$$
\hat{\mathbf{a}}_{0}(\mathbf{x})=\int d \mathbf{x}^{\prime} \frac{\tilde{\hat{\sigma}}\left(\mathbf{x}^{\prime}\right)}{\left|\mathbf{x}-\mathbf{x}^{\prime}\right|}
$$

Next it is easy to verify that

$$
\left[\hat{\eta}_{\alpha}(\mathbf{X}), \hat{\eta}_{\beta}^{+}\left(\mathbf{X}^{\prime}\right)\right]=\delta_{\alpha \beta} \delta\left(\mathbf{X}-\mathbf{X}^{\prime}\right), \quad \sum_{\alpha} \varphi_{\alpha}^{*}(\mathbf{x}) \varphi_{\alpha}\left(\mathbf{x}^{\prime}\right)=\delta\left(\mathbf{x}-\mathbf{x}^{\prime}\right)
$$

lead to the following commutation relation:

$$
\left[\hat{\varphi}(\mathbf{x}, \mathbf{X}), \hat{\varphi}^{+}\left(\mathbf{x}^{\prime}, \mathbf{X}^{\prime}\right)\right]=\delta\left(\mathbf{x}-\mathbf{x}^{\prime}\right) \delta\left(\mathbf{X}-\mathbf{X}^{\prime}\right)
$$

where $\hat{\varphi}(\mathbf{x}, \mathbf{X}) \equiv \hat{\varphi}\left(\mathbf{x}_{1}, \mathbf{x}_{2}\right)$ is determined by (17). Hereinafter we assume that a set of wave functions $\varphi_{\alpha}\left(\mathbf{x}^{\prime}\right)$ is complete. Therefore this set accounts for the wave functions of continuous spectrum. In addition, if we introduce the operator

$$
\hat{n}(\mathbf{x}, \mathbf{X})=\hat{\varphi}^{+}(\mathbf{x}, \mathbf{X}) \hat{\varphi}(\mathbf{x}, \mathbf{X})
$$

then

$$
\left[\hat{n}(\mathbf{x}, \mathbf{X}), \hat{n}\left(\mathbf{x}^{\prime}, \mathbf{X}^{\prime}\right)\right]=0
$$

Thus, it is clear that the Hamiltonian $\tilde{\hat{\mathcal{H}}}_{\text {int }}$, which describes the Coulomb interaction between particles and bound states meets the commutation relation

$$
\left[\tilde{\hat{\mathcal{H}}}_{i n t}, \tilde{\hat{\rho}}_{i}(\mathbf{x})\right]=0 .
$$

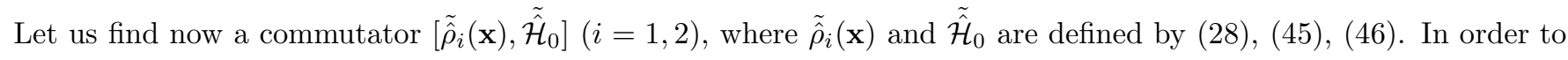
do this we consider $\tilde{\hat{\rho}}_{1}(\mathbf{x})$ in the form

$$
\tilde{\hat{\rho}}_{1}(\mathbf{x})=\tilde{\hat{\rho}}_{1}^{\prime}(\mathbf{x})+\tilde{\hat{\rho}}_{1}^{\prime \prime}(\mathbf{x}),
$$

where

$$
\tilde{\hat{\rho}}_{1}^{\prime}(\mathbf{x})=\hat{\chi}_{1}^{+}(\mathbf{x}) \hat{\chi}_{1}(\mathbf{x}), \quad \tilde{\hat{\rho}}_{1}^{\prime \prime}(\mathbf{x})=\int d \mathbf{y} d \mathbf{Y} \delta\left(\mathbf{x}-\mathbf{Y}-\frac{m_{2}}{M} \mathbf{y}\right) \hat{\varphi}^{+}(\mathbf{y}, \mathbf{Y}) \hat{\varphi}(\mathbf{y}, \mathbf{Y})
$$

Then in accordance with the commutation relations for the field operators $\hat{\chi}_{i}(\mathbf{x})$,

$$
\left[\hat{\chi}_{i}(\mathbf{x}), \hat{\chi}_{j}^{+}\left(\mathbf{x}^{\prime}\right)\right]=\delta_{i j} \delta\left(\mathbf{x}-\mathbf{x}^{\prime}\right)
$$

one gets

$$
\left[\tilde{\hat{\rho}}_{1}^{\prime}(\mathbf{x}), \int d \mathbf{x}^{\prime} \sum_{j=1}^{2} \frac{1}{2 m_{j}} \frac{\partial \hat{\chi}_{j}^{+}\left(\mathbf{x}^{\prime}\right)}{\partial \mathbf{x}^{\prime}} \frac{\partial \hat{\chi}_{j}\left(\mathbf{x}^{\prime}\right)}{\partial \mathbf{x}^{\prime}}\right]=-\frac{i}{2 m_{1}} \frac{\partial}{\partial x_{k}}\left(\hat{\chi}_{1}^{+}(\mathbf{x}) \frac{\partial \hat{\chi}_{1}(\mathbf{x})}{\partial x_{k}}-\frac{\partial \hat{\chi}_{1}^{+}(\mathbf{x})}{\partial x_{k}} \hat{\chi}_{1}(\mathbf{x})\right)
$$


Next according to (30) we have

$$
\begin{aligned}
i\left[\tilde{\hat{\rho}}_{1}^{\prime \prime}(\mathbf{x}), \tilde{\hat{\mathcal{H}}}_{0}\right] & =\frac{1}{2 \mu} \int d \mathbf{y} d \mathbf{Y} \delta\left(\mathbf{x}-\mathbf{Y}-\frac{m_{2}}{M} \mathbf{y}\right) \frac{\partial}{\partial y_{k}}\left(-\hat{\varphi}^{+}(\mathbf{y}, \mathbf{Y}) \frac{\partial \hat{\varphi}(\mathbf{y}, \mathbf{Y})}{\partial y_{k}}+\frac{\partial \hat{\varphi}^{+}(\mathbf{y}, \mathbf{Y})}{\partial y_{k}} \hat{\varphi}(\mathbf{y}, \mathbf{Y})\right) \\
& +\frac{1}{2 M} \int d \mathbf{y} d \mathbf{Y} \delta\left(\mathbf{x}-\mathbf{Y}-\frac{m_{2}}{M} \mathbf{y}\right) \frac{\partial}{\partial Y_{k}}\left(-\hat{\varphi}^{+}(\mathbf{y}, \mathbf{Y}) \frac{\partial \hat{\varphi}(\mathbf{y}, \mathbf{Y})}{\partial Y_{k}}+\frac{\partial \hat{\varphi}^{+}(\mathbf{y}, \mathbf{Y})}{\partial Y_{k}} \hat{\varphi}(\mathbf{y}, \mathbf{Y})\right) .
\end{aligned}
$$

Bearing in mind

$$
-\frac{\partial}{\partial y_{k}} \delta\left(\mathbf{x}-\mathbf{Y}-\frac{m_{2}}{M} \mathbf{y}\right)=\frac{m_{2}}{M} \frac{\partial}{\partial x_{k}} \delta\left(\mathbf{x}-\mathbf{Y}-\frac{m_{2}}{M} \mathbf{y}\right), \quad-\frac{\partial}{\partial Y_{k}} \delta\left(\mathbf{x}-\mathbf{Y}-\frac{m_{2}}{M} \mathbf{y}\right)=\frac{\partial}{\partial x_{k}} \delta\left(\mathbf{x}-\mathbf{Y}-\frac{m_{2}}{M} \mathbf{y}\right)
$$

and taking into account the definitions of $\tilde{\hat{\rho}}_{i}, \tilde{\hat{\pi}}_{i}(i=1,2)$, one finds

$$
i\left[\tilde{\hat{\rho}}_{1}(\mathbf{x}), \tilde{\hat{\mathcal{H}}}_{0}+\tilde{\hat{\mathcal{H}}}_{i n t}\right]=\frac{1}{m_{1}} \operatorname{div} \tilde{\hat{\boldsymbol{\pi}}}_{1}(\mathbf{x}) .
$$

Similarly,

$$
i\left[\tilde{\hat{\rho}}_{2}(\mathbf{x}), \tilde{\hat{\mathcal{H}}}_{0}+\tilde{\hat{\mathcal{H}}}_{i n t}\right]=\frac{1}{m_{2}} \operatorname{div} \tilde{\hat{\boldsymbol{\pi}}}_{2}(\mathbf{x}) .
$$

Let us find now a commutator $\left[\tilde{\hat{\rho}}_{i}\left(\mathbf{x}^{\prime}\right), \tilde{\hat{\pi}}_{k}(\mathbf{x})\right],(i, k=1,2)$. With the use of (27) we have

$$
\left[\tilde{\hat{\rho}}_{i}^{\prime \prime}\left(\mathbf{x}^{\prime}\right), \tilde{\hat{\boldsymbol{\pi}}}_{k}(\mathbf{x})\right]=i \delta_{i k} \tilde{\hat{\rho}}_{i}^{\prime \prime}(\mathbf{x}) \frac{\partial}{\partial \mathbf{x}} \delta\left(\mathbf{x}-\mathbf{x}^{\prime}\right) .
$$

Noting also that

$$
\left[\tilde{\hat{\rho}}_{i}^{\prime}\left(\mathbf{x}^{\prime}\right), \tilde{\hat{\boldsymbol{\pi}}}_{k}(\mathbf{x})\right]=i \delta_{i k} \tilde{\hat{\rho}}_{i}^{\prime}(\mathbf{x}) \frac{\partial}{\partial \mathbf{x}} \delta\left(\mathbf{x}-\mathbf{x}^{\prime}\right),
$$

we get finally

$$
\left[\tilde{\hat{\rho}}_{i}\left(\mathbf{x}^{\prime}\right), \tilde{\hat{\boldsymbol{\pi}}}_{k}(\mathbf{x})\right]=i \delta_{i k} \tilde{\hat{\rho}}_{i}(\mathbf{x}) \frac{\partial}{\partial \mathbf{x}} \delta\left(\mathbf{x}-\mathbf{x}^{\prime}\right) .
$$

These commutation relations along with (49) result in

$$
\left[\tilde{\hat{\mathbf{j}}}_{0}(\mathbf{x}), \tilde{\hat{\sigma}}\left(\mathbf{x}^{\prime}\right)\right]=-i \sum_{i=1}^{2} \frac{e_{i}}{m_{i}} \tilde{\hat{\sigma}}_{i}\left(\mathbf{x}^{\prime}\right) \frac{\partial}{\partial \mathbf{x}} \delta\left(\mathbf{x}-\mathbf{x}^{\prime}\right) .
$$

Thus, according to (52), (55), (56) we have

$$
\dot{\hat{\sigma}}(\mathbf{x})=i[\tilde{\hat{\mathcal{H}}}(t), \tilde{\hat{\sigma}}(\mathbf{x})]=\operatorname{div} \tilde{\hat{\mathbf{J}}}(\mathbf{x}, t) .
$$

The definitions (52), (53) of the longitudinal component of the electric field along with the above formula give

$$
\dot{\hat{\mathbf{e}}}^{l}(\mathbf{x}) \equiv i\left[\tilde{\hat{\mathcal{H}}}(t), \hat{\mathbf{e}}^{l}(\mathbf{x})\right]=\frac{\partial}{\partial \mathbf{x}} \operatorname{div} \int d \mathbf{x}^{\prime} \frac{\tilde{\hat{\mathbf{J}}}\left(\mathbf{x}^{\prime}, t\right)}{\left|\mathbf{x}-\mathbf{x}^{\prime}\right|} .
$$

Let us find the equation of motion for $\hat{\mathbf{e}}^{t}$. The use of commutation relations for creation $\hat{C}_{\mathbf{k} \lambda}^{+}$and annihilation $\hat{C}_{\mathbf{k} \lambda}$ operators of photons,

$$
\left[\hat{C}_{\mathbf{k} \lambda}, \hat{C}_{\mathbf{k}^{\prime} \lambda^{\prime}}^{+}\right]=\delta_{\mathbf{k k}^{\prime}} \delta_{\lambda \lambda^{\prime}}
$$


lead to

$$
\begin{gathered}
\hat{\mathbf{e}}^{t}(\mathbf{x})=-i[\tilde{\hat{\mathcal{H}}}(t), \hat{\mathbf{a}}(\mathbf{x})]=i \sum_{\mathbf{k}} \sum_{\lambda=1}^{2}\left(\frac{2 \pi \omega_{k}}{\mathcal{V}}\right)^{1 / 2}\left(\mathbf{e}_{\mathbf{k}}^{(\lambda)} \hat{C}_{\mathbf{k} \lambda} e^{i \mathbf{k} \mathbf{x}}-h . c .\right), \\
\dot{\hat{\mathbf{e}}}^{t}(\mathbf{x})=-i\left[\tilde{\hat{\mathcal{H}}}(t), \hat{\mathbf{e}}^{t}(\mathbf{x})\right]=\operatorname{rot} \hat{\mathbf{h}}(\mathbf{x})+i\left[\hat{\hat{V}}(t), \hat{\mathbf{e}}^{t}(\mathbf{x})\right],
\end{gathered}
$$

and also

$$
\left[\hat{a}_{i}(\mathbf{x}), \hat{e}_{s}^{t}\left(\mathbf{x}^{\prime}\right)\right]=-4 \pi i \delta_{i s} \delta\left(\mathbf{x}-\mathbf{x}^{\prime}\right)-i \frac{\partial^{2}}{\partial x_{i} \partial x_{s}} \frac{1}{\left|\mathbf{x}-\mathbf{x}^{\prime}\right|}
$$

This formula and the definition of $\tilde{\hat{V}}(t)$ allows us to find the commutator:

$$
i\left[\tilde{\hat{V}}(t), \hat{\mathbf{e}}^{t}(\mathbf{x})\right]=-4 \pi \tilde{\hat{\mathbf{J}}}(\mathbf{x}, t)-\frac{\partial}{\partial \mathbf{x}} \operatorname{div} \int d \mathbf{x}^{\prime} \frac{\tilde{\hat{\mathbf{J}}}\left(\mathbf{x}^{\prime}, t\right)}{\left|\mathbf{x}-\mathbf{x}^{\prime}\right|} .
$$

Therefore, it follows from (57), (58) that

$$
\dot{\hat{\mathbf{e}}}(\mathbf{x})=\operatorname{rot} \hat{\mathbf{h}}(\mathbf{x})-4 \pi \tilde{\hat{\mathbf{J}}}(\mathbf{x}, t), \quad \dot{\hat{\mathbf{h}}}(\mathbf{x}) \equiv i[\hat{\hat{\mathcal{H}}}(t), \hat{\mathbf{h}}(\mathbf{x})]=i\left[\hat{\mathcal{H}}_{f}, \hat{\mathbf{h}}(\mathbf{x})\right]
$$

Upon calculating this commutator with the use of (151), one obtains

$$
\dot{\hat{\mathbf{h}}}(\mathbf{x})=-\operatorname{rot} \hat{\mathbf{e}}(\mathbf{x})
$$

In addition, (51), (52) give $\operatorname{div} \hat{\mathbf{h}}(\mathbf{x})=0, \operatorname{div} \hat{\mathbf{e}}(\mathbf{x})=4 \pi \tilde{\hat{\sigma}}(\mathbf{x})$. These equations along with (59), (60) represent the Maxwell-Lorentz equations for the operators of electromagnetic field

$$
\begin{gathered}
2 \dot{\hat{\mathbf{e}}}(\mathbf{x})=\operatorname{rot} \hat{\mathbf{h}}(\mathbf{x})-4 \pi \tilde{\hat{\mathbf{J}}}(\mathbf{x}, t), \quad \operatorname{div} \hat{\mathbf{e}}(\mathbf{x})=4 \pi \tilde{\hat{\sigma}}(\mathbf{x}), \\
\dot{\hat{\mathbf{h}}}(\mathbf{x})=-\operatorname{rot} \hat{\mathbf{e}}(\mathbf{x}), \quad \operatorname{div} \hat{\mathbf{h}}(\mathbf{x})=0 .
\end{gathered}
$$

Notice that (46), (51), (58) result in

$$
\hat{\mathcal{H}}_{f}=\sum_{\mathbf{k}} \sum_{\lambda=1}^{2} \omega_{k} \hat{C}_{\mathbf{k} \lambda}^{+} \hat{C}_{\mathbf{k} \lambda}=\frac{1}{8 \pi} \int d \mathbf{x}\left(\mathbf{e}^{t^{2}}+\mathbf{h}^{2}\right),
$$

where we neglect the unessential term $\sum_{\mathbf{k} \lambda} \frac{1}{2} \omega_{k}$.

Up to now we used the Schroedinger representation, in which the Schroedinger equation has the form

$$
i \frac{\partial \Phi(t)}{\partial t}=\tilde{\hat{\mathcal{H}}}(t) \Phi(t)
$$

In this representation a dot above the certain operator $\dot{\hat{b}}(\mathbf{x})$ (see (152)) does not mean the differentiation with respect to time. It means the following:

$$
\dot{\hat{b}}(\mathbf{x})=i\left[\tilde{\hat{\mathcal{H}}}\left(\underline{\hat{\chi}}\left(\mathbf{x}^{\prime}\right), t\right), \hat{b}(\mathbf{x})\right]
$$

Here the Hamiltonian $\tilde{\hat{\mathcal{H}}}$ depends on dynamic variables $\underline{\hat{\chi}}\left(\mathbf{x}^{\prime}\right)$ (these variables unite $\hat{\chi}$ and $\hat{\eta}$ ) and time $t$. Introducing the unitary operator $\hat{S}(t, 0)$,

$$
i \frac{\partial \hat{S}(t, 0)}{\partial t}=\tilde{\hat{\mathcal{H}}}\left(\underline{\hat{\chi}}\left(\mathbf{x}^{\prime}\right), t\right) \hat{S}(t, 0), \quad \hat{S}(0,0)=1
$$


we can write the solution of the equation for $\Phi(t)$ in the form,

$$
\Phi(t)=\hat{S}(t, 0) \Phi(0)
$$

In the Heisenberg representation the vector $\Phi(0)=\Psi$ is taken as a time-independent state vector. Then, the time dependent Heisenberg operators

$$
\underline{\hat{\chi}}(\mathbf{x}, t) \equiv \underline{\hat{\chi}}(x)=\hat{S}^{+}(t, 0) \underline{\hat{\chi}}(\mathbf{x}) \hat{S}(t, 0)
$$

satisfy the equation

$$
i \frac{\partial \underline{\hat{\chi}}(x)}{\partial t}=[\underline{\hat{\chi}}(x), \hat{\hat{\mathcal{H}}}(\underline{\hat{\chi}}(x), t)]
$$

Since the external fields $\mathbf{H}^{(e)}, \mathbf{E}^{(e)}$ satisfy the Maxwell equations,

$$
\begin{gathered}
\frac{\partial \mathbf{H}^{(e)}}{\partial t}=-\operatorname{rot} \mathbf{E}^{(e)}, \quad \operatorname{div} \mathbf{H}^{(e)}=0, \\
\frac{\partial \mathbf{E}^{(e)}}{\partial t}=\operatorname{rot} \mathbf{H}^{(e)}-4 \pi \mathbf{J}^{(e)},
\end{gathered}
$$

the operators of total fields $\hat{\mathbf{E}}, \hat{\mathbf{H}}$ in the Heisenberg representation satisfy, in accordance with (62), the equations

$$
\begin{gathered}
\frac{\partial \hat{\mathbf{H}}}{\partial t}=-\operatorname{rot} \hat{\mathbf{E}}, \quad \operatorname{div} \hat{\mathbf{H}}=0, \\
\frac{\partial \hat{\mathbf{E}}}{\partial t}=\operatorname{rot} \hat{\mathbf{H}}-4 \pi\left(\tilde{\hat{\mathbf{J}}}+\mathbf{J}^{(e)}\right), \quad \operatorname{div} \hat{\mathbf{E}}=4 \pi\left(\hat{\hat{\sigma}}+\sigma^{(e)}\right),
\end{gathered}
$$

where $\mathbf{J}^{(e)}$ and $\sigma^{(e)}$ are the extrinsic current and charge densities.

Let us write out the expressions for the charge density operator $\tilde{\hat{\sigma}}$ and current density operator $\tilde{\hat{\mathbf{J}}}$ in terms of creation and annihilation operators of particles and bound states. Bearing in mind (50) we have

$$
\begin{gathered}
\tilde{\hat{\sigma}}(\mathbf{x})=\sum_{i=1}^{2} e_{i} \tilde{\hat{\rho}}_{i}(\mathbf{x}), \\
\tilde{\hat{\mathbf{J}}}(\mathbf{x})=-\hat{\mathbf{A}}(\mathbf{x}) \sum_{i=1}^{2} \frac{e_{i}^{2}}{m_{i}} \tilde{\hat{\rho}}_{i}(\mathbf{x})+\sum_{i=1}^{2} \frac{e_{i}}{m_{i}} \tilde{\hat{\boldsymbol{\pi}}}_{i}(\mathbf{x}),
\end{gathered}
$$

where the particle density operators $\tilde{\hat{\rho}}_{i}(\mathbf{x})$ are defined by (28) and the momentum density operators $\tilde{\hat{\boldsymbol{\pi}}}_{i}(\mathbf{x})$ by (27). The first terms in expressions for $\tilde{\hat{\rho}}_{i}(\mathbf{x})$ and $\tilde{\hat{\boldsymbol{\pi}}}_{i}(\mathbf{x})$ define the contribution of particles, whereas the second terms define the contribution of bound states (see (27), (28)).

Let us find now the equations of motion for the field operators of particles $\hat{\chi}_{i}(\mathbf{x})$ and bound states $\hat{\varphi}(\mathbf{x}, \mathbf{y})$. In the Heisenberg representation these equations are obtained from the general formulas (62). Indeed, it follows from (38)- (40) that

$$
\begin{gathered}
{\left[\hat{\chi}_{1}(\mathbf{x}), \tilde{\hat{\mathcal{H}}}_{i n t}\left(\hat{\chi}\left(\mathbf{x}^{\prime}\right)\right)\right]=\left\{\int d \mathbf{x}_{2} \nu_{11}\left(\mathbf{x}-\mathbf{x}_{2}\right) \tilde{\hat{\rho}}_{1}\left(\mathbf{x}_{2}\right)+\int d \mathbf{x}_{2} \nu_{12}\left(\mathbf{x}-\mathbf{x}_{2}\right) \tilde{\hat{\rho}}_{2}\left(\mathbf{x}_{2}\right)\right\} \hat{\chi}_{1}(\mathbf{x}),} \\
{\left[\hat{\chi}_{2}(\mathbf{x}), \hat{\hat{\mathcal{H}}}_{i n t}\left(\hat{\chi}\left(\mathbf{x}^{\prime}\right)\right)\right]=\left\{\int d \mathbf{x}_{1} \nu_{22}\left(\mathbf{x}-\mathbf{x}_{1}\right) \tilde{\hat{\rho}}_{2}\left(\mathbf{x}_{1}\right)+\int d \mathbf{x}_{2} \nu_{21}\left(\mathbf{x}-\mathbf{x}_{1}\right) \tilde{\hat{\rho}}_{1}\left(\mathbf{x}_{1}\right)\right\} \hat{\chi}_{2}(\mathbf{x}),} \\
{\left[\hat{\varphi}(\mathbf{x}, \mathbf{y}), \tilde{\hat{\mathcal{H}}}_{i n t}\left(\hat{\chi}\left(\mathbf{x}^{\prime}\right)\right)\right]=\left\{\int d \mathbf{x}_{2}\left(\nu_{11}\left(\mathbf{x}-\mathbf{x}_{2}\right)+\nu_{12}\left(\mathbf{y}-\mathbf{x}_{2}\right)\right) \tilde{\hat{\rho}}_{1}\left(\mathbf{x}_{2}\right)+\right.} \\
\left.\int d \mathbf{x}_{2}\left(\nu_{22}\left(\mathbf{y}-\mathbf{x}_{2}\right)+\nu_{21}\left(\mathbf{x}-\mathbf{x}_{2}\right)\right) \tilde{\hat{\rho}}_{2}\left(\mathbf{x}_{2}\right)\right\} \hat{\varphi}(\mathbf{x}, \mathbf{y}) .
\end{gathered}
$$


These formulas allow to find the equations of motion (62) for $\hat{\chi}_{1}(\mathbf{x}), \hat{\chi}_{2}(\mathbf{x})$ in the Heisenberg representation,

$$
\begin{aligned}
& i \dot{\hat{\chi}}_{1}(\mathbf{x}, t)=\left\{-\frac{1}{2 m_{1}}\left(\frac{\partial}{\partial \mathbf{x}}-i e_{1} \hat{\mathbf{A}}(\mathbf{x}, t)\right)^{2}+e_{1} \varphi^{(e)}(\mathbf{x}, t)+\hat{v}_{1}(\mathbf{x}, t)\right\} \hat{\chi}_{1}(\mathbf{x}, t), \\
& i \dot{\hat{\chi}}_{2}(\mathbf{x}, t)=\left\{-\frac{1}{2 m_{2}}\left(\frac{\partial}{\partial \mathbf{x}}-i e_{2} \hat{\mathbf{A}}(\mathbf{x}, t)\right)^{2}+e_{2} \varphi^{(e)}(\mathbf{x}, t)+\hat{v}_{2}(\mathbf{x}, t)\right\} \hat{\chi}_{2}(\mathbf{x}, t) .
\end{aligned}
$$

It is easy to find also the equation of motion for the field operators $\hat{\varphi}(\mathbf{x}, \mathbf{y})$ of bound states,

$$
\begin{aligned}
i \dot{\hat{\varphi}}\left(\mathbf{x}_{1}, \mathbf{x}_{2}, t\right) & =\sum_{\alpha} \varepsilon_{\alpha} \hat{\eta}_{\alpha}(\mathbf{X}, t) \varphi_{\alpha}(\mathbf{x})+\left\{-\frac{1}{2 m_{1}}\left(\frac{\partial}{\partial \mathbf{x}_{1}}-i e_{1} \hat{\mathbf{A}}\left(\mathbf{x}_{1}, t\right)\right)^{2}-\frac{1}{2 m_{2}}\left(\frac{\partial}{\partial \mathbf{x}_{2}}-i e_{2} \hat{\mathbf{A}}\left(\mathbf{x}_{2}, t\right)\right)^{2}\right. \\
& \left.+e_{1} \varphi^{(e)}\left(\mathbf{x}_{1}, t\right)+e_{2} \varphi^{(e)}\left(\mathbf{x}_{2}, t\right)+\hat{v}\left(\mathbf{x}_{1}, \mathbf{x}_{2}, t\right)\right\} \hat{\varphi}\left(\mathbf{x}_{1}, \mathbf{x}_{2}, t\right)
\end{aligned}
$$

Here

$$
\begin{gathered}
\hat{v}_{1}(\mathbf{x}, t)=\int d \mathbf{x}_{2} \nu_{11}\left(\mathbf{x}-\mathbf{x}_{2}\right) \tilde{\hat{\rho}}_{1}\left(\mathbf{x}_{2}, t\right)+\int d \mathbf{x}_{2} \nu_{12}\left(\mathbf{x}-\mathbf{x}_{2}\right) \tilde{\hat{\rho}}_{2}\left(\mathbf{x}_{2}, t\right) \\
\hat{v}_{2}(\mathbf{x}, t)=\int d \mathbf{x}_{1} \nu_{22}\left(\mathbf{x}-\mathbf{x}_{1}\right) \tilde{\hat{\rho}}_{2}\left(\mathbf{x}_{1}, t\right)+\int d \mathbf{x}_{1} \nu_{21}\left(\mathbf{x}-\mathbf{x}_{1}\right) \tilde{\hat{\rho}}_{1}\left(\mathbf{x}_{1}, t\right), \\
\hat{v}\left(\mathbf{x}_{1}, \mathbf{x}_{2}, t\right)=\int d \mathbf{x}^{\prime}\left(\nu_{11}\left(\mathbf{x}_{1}-\mathbf{x}^{\prime}\right)+\nu_{12}\left(\mathbf{x}_{2}-\mathbf{x}^{\prime}\right)\right) \tilde{\hat{\rho}}_{1}\left(\mathbf{x}^{\prime}, t\right)+\int d \mathbf{x}^{\prime}\left(\nu_{22}\left(\mathbf{x}_{2}-\mathbf{x}^{\prime}\right)+\nu_{21}\left(\mathbf{x}_{1}-\mathbf{x}^{\prime}\right)\right) \tilde{\hat{\rho}}_{2}\left(\mathbf{x}^{\prime}, t\right) .
\end{gathered}
$$

The operators $\hat{\eta}_{\alpha}(\mathbf{X}, t)$ related to $\hat{\varphi}\left(\mathbf{x}_{1}, \mathbf{x}_{2}, t\right)$ by $\hat{\varphi}\left(\mathbf{x}_{1}, \mathbf{x}_{2}, t\right)=\varphi_{\beta}(\mathbf{x}) \hat{\eta}_{\beta}(\mathbf{X}, t)$.

The equations of motion (63), (65) are gauge-invariant. Indeed, under the following transformations of fields $\hat{\chi}_{i}, \hat{\varphi}$ :

$$
\begin{gathered}
\hat{\chi}_{1}(\mathbf{x}, t) \rightarrow \hat{\chi}_{1}^{\prime}(\mathbf{x}, t)=e^{i e_{1} \alpha(\mathbf{x}, t)} \hat{\chi}_{1}(\mathbf{x}, t), \quad \hat{\chi}_{2}(\mathbf{x}, t) \rightarrow \hat{\chi}_{2}^{\prime}(\mathbf{x}, t)=e^{i e_{2} \alpha(\mathbf{x}, t)} \hat{\chi}_{2}(\mathbf{x}, t) \\
\hat{\varphi}(\mathbf{x}, \mathbf{y}, t) \rightarrow \hat{\varphi}^{\prime}(\mathbf{x}, \mathbf{y}, t)=e^{i e_{1} \alpha(\mathbf{x}, t)+i e_{2} \alpha(\mathbf{y}, t)} \hat{\varphi}(\mathbf{x}, \mathbf{y}, t)
\end{gathered}
$$

and electromagnetic field,

$$
\hat{\mathbf{A}}(\mathbf{x}, t) \rightarrow \hat{\mathbf{A}}^{\prime}(\mathbf{x}, t)=\hat{\mathbf{A}}(\mathbf{x}, t)-\frac{\partial}{\partial \mathbf{x}} \alpha(\mathbf{x}, t), \quad \varphi^{(e)}(\mathbf{x}, t) \rightarrow \varphi^{(e)^{\prime}}(\mathbf{x}, t)=\varphi^{(e)}(\mathbf{x})-\frac{\partial \alpha(\mathbf{x}, t)}{\partial t}
$$

the operators $\tilde{\hat{\rho}}_{i}(\mathbf{x})$ are invariant,

$$
\tilde{\hat{\rho}}_{i}(\mathbf{x}, t) \rightarrow \tilde{\hat{\rho}}_{i}^{\prime}(\mathbf{x}, t)=\tilde{\hat{\rho}}_{i}(\mathbf{x}, t)
$$

whereas $\tilde{\hat{\boldsymbol{\pi}}}_{i}(\mathbf{x})$ transform (see $(\underline{65})$ ), by the law

$$
\tilde{\hat{\boldsymbol{\pi}}}_{i}(\mathbf{x}, t) \rightarrow \tilde{\hat{\boldsymbol{\pi}}}_{i}^{\prime}(\mathbf{x}, t)=\tilde{\hat{\boldsymbol{\pi}}}_{i}(\mathbf{x}, t)+e_{i} \tilde{\hat{\rho}}_{i}(\mathbf{x}, t) \frac{\partial}{\partial \mathbf{x}} \alpha(\mathbf{x}, t)
$$

As a result we have (see 64)

$$
\tilde{\hat{\mathbf{J}}}(\mathbf{x}, t) \rightarrow \tilde{\hat{\mathbf{J}}}^{\prime}(\mathbf{x}, t)=\tilde{\hat{\mathbf{J}}}(\mathbf{x}, t), \quad \tilde{\hat{\mathcal{H}}} \rightarrow \tilde{\hat{\mathcal{H}}}^{\prime}=\tilde{\hat{\mathcal{H}}}
$$

that provides a gauge invariance of the Maxwell-Lorentz equations (63). Next noting that

$$
\left(\frac{\partial}{\partial \mathbf{x}}-i e_{i} \hat{\mathbf{A}}(\mathbf{x}, t)\right) \hat{\chi}_{i}(\mathbf{x}, t) \rightarrow\left(\frac{\partial}{\partial \mathbf{x}}-i e_{i} \hat{\mathbf{A}}^{\prime}(\mathbf{x}, t)\right) \hat{\chi}_{i}^{\prime}(\mathbf{x}, t)=e^{i e_{i} \alpha(\mathbf{x}, t)}\left(\frac{\partial}{\partial \mathbf{x}}-i e_{i} \hat{\mathbf{A}}(\mathbf{x}, t)\right) \hat{\chi}_{i}(\mathbf{x}, t)
$$


we make sure of gauge-invariance of the equations of motion for the field operators of particles $\hat{\chi}_{i}(\mathbf{x})$. Taking into account the transformation law for the field operators of bound states $\hat{\varphi}(\mathbf{x}, \mathbf{y})$, it is easy to prove a gauge invariance of the equation of motion for $\hat{\varphi}(\mathbf{x}, \mathbf{y})$.

Since $\hat{\varphi}\left(\mathbf{x}_{1}, \mathbf{x}_{2}, t\right)=\varphi_{\alpha}(\mathbf{x}) \hat{\eta}_{\alpha}(\mathbf{X}, t)$, we have under the gauge transformation (66)),

$$
\hat{\varphi}^{\prime}\left(\mathbf{x}_{1}, \mathbf{x}_{2}, t\right)=\varphi_{\alpha}(\mathbf{x}) \hat{\eta}_{\alpha}^{\prime}(\mathbf{X}, t)
$$

where $\mathbf{x}_{1}=\mathbf{X}+\left(m_{2} / M\right) \mathbf{x}, \mathbf{x}_{2}=\mathbf{X}-\left(m_{1} / M\right) \mathbf{x}$. Hence, we come to the transformation law for the operators $\hat{\eta}_{\alpha}(\mathbf{X})$ : $\hat{\eta}_{\alpha}(\mathbf{X}, t) \rightarrow \hat{\eta}_{\alpha}^{\prime}(\mathbf{X}, t)=\sum_{\beta} L_{\alpha \beta}(\mathbf{X}, t) \hat{\eta}_{\beta}(\mathbf{X}, t), \quad L_{\alpha \beta}(\mathbf{X}, t)=\int d \mathbf{x} \varphi_{\alpha}^{*}(\mathbf{x}) e^{i e_{1} \alpha\left[\mathbf{X}+\left(m_{2} / M\right) \mathbf{x}, t\right]+i e_{2} \alpha\left[\mathbf{X}-\left(m_{1} / M\right) \mathbf{x}, t\right]} \varphi_{\beta}(\mathbf{x})$

In conclusion of this section let us note that $\tilde{\hat{\psi}}_{i}(\mathbf{x})$ transform under the gauge transformation as well as $\hat{\chi}_{i}(\mathbf{x})$ :

$$
\tilde{\hat{\psi}}_{i}(\mathbf{x}, t) \rightarrow \tilde{\hat{\psi}}_{i}^{\prime}(\mathbf{x}, t)=e^{i e_{i} \alpha(\mathbf{x}, t)} \tilde{\hat{\psi}}_{i}(\mathbf{x}, t)
$$

\section{THE PROCESS OF SPONTANEOUS RADIATION BY ATOM}

In this section we employ the obtained formulas in order to find the probability distribution of spontaneous radiation of an excited atom. To this end we use the general formulas for the scattering matrix $\hat{S}$ in terms of the T-operator:

$$
\begin{gathered}
\hat{S}=1-2 \pi i \int_{-\infty}^{\infty} d E \delta\left(E-\hat{\mathcal{H}}_{0}\right) \hat{T}^{(+)}(E) \delta\left(E-\hat{\mathcal{H}}_{0}\right), \\
\hat{T}^{(+)}(E)=\lim _{\eta \rightarrow+0} \hat{T}(E+i \eta),
\end{gathered}
$$

where $\hat{T}(z)$ is found from the equation

$$
\hat{T}(z)=\hat{V}+\hat{V} \hat{R}_{0} \hat{T}(z), \quad \hat{R}_{0}=\frac{1}{z-\hat{\mathcal{H}}_{0}} .
$$

In the first order of the perturbative approach over charge we have

$$
\langle f|\hat{T}(z)| i\rangle \approx\langle f|\hat{V}| i\rangle
$$

where

$$
|i\rangle=\hat{\eta}_{\alpha \mathbf{p}}^{+}|0\rangle, \quad|f\rangle=\hat{C}_{\mathbf{k} \lambda}^{+} \hat{\eta}_{\alpha \mathbf{p}}^{+}|0\rangle .
$$

Here $|i\rangle$ is the initial state (in which atom is in the state $\alpha, \mathbf{p}$ ) and $|f\rangle$ is the final state (in which atom is in the state $\alpha^{\prime}, \mathbf{p}^{\prime}$ and photon is in the state $\lambda, \mathbf{k}$ ). According to (48), (150) we can take $\hat{V}$ in the following form:

$$
\tilde{\hat{V}}=-\int d \mathbf{x} \hat{\mathbf{a}}(\mathbf{x}) \tilde{\hat{\mathbf{j}}}(\mathbf{x})
$$

where the current of bound states is defined by

$$
\tilde{\hat{\mathbf{j}}}(\mathbf{x})=\frac{e_{1}}{m_{1}} \tilde{\boldsymbol{\pi}}_{1}(\mathbf{x})+\frac{e_{2}}{m_{2}} \tilde{\hat{\pi}}_{2}(\mathbf{x})
$$


By using of (48), (150) we find

$$
\langle f|\tilde{\hat{V}}| i\rangle=-\left(\frac{2 \pi}{\mathcal{V} \omega_{k}}\right)^{1 / 2} \mathbf{e}_{\mathbf{k}}^{(\lambda)^{*}} \int d \mathbf{x} e^{-i \mathbf{k x}}\left\langle\alpha^{\prime} \mathbf{p}^{\prime}|\tilde{\hat{\mathbf{j}}}(\mathbf{x})| \alpha \mathbf{p}\right\rangle .
$$

The formulas (69), (27) lead to

$$
\tilde{\hat{\mathbf{j}}}(\mathbf{k}) \equiv \int d \mathbf{x} e^{-i \mathbf{k x}} \tilde{\hat{\mathbf{j}}}(\mathbf{x})=\sum_{\mathbf{p}_{1}, \mathbf{p}_{2}} \sum_{\alpha_{1}, \alpha_{2}} \hat{\eta}_{\alpha_{1} \mathbf{p}_{1}}^{+} \hat{\eta}_{\alpha_{2} \mathbf{p}_{2}}\left(\mathbf{g}_{\alpha_{1} \alpha_{2}}(\mathbf{k})+\frac{\mathbf{p}_{1}+\mathbf{p}_{2}}{2 M} g_{\alpha_{1} \alpha_{2}}(\mathbf{k})\right) \Delta\left(\mathbf{p}_{1}-\mathbf{p}_{2}+\mathbf{k}\right)
$$

and, consequently,

$$
\langle f|\tilde{\hat{V}}| i\rangle=-\left(\frac{2 \pi}{\mathcal{V} \omega_{k}}\right)^{1 / 2} \mathbf{e}_{\mathbf{k}}^{(\lambda) *}\left(\mathbf{g}_{\alpha \alpha^{\prime}}(\mathbf{k})+\frac{\mathbf{p}+\mathbf{p}^{\prime}}{2 M} g_{\alpha \alpha^{\prime}}(\mathbf{k})\right) \Delta\left(\mathbf{p}-\mathbf{p}^{\prime}-\mathbf{k}\right), \quad \mathbf{k}=\mathbf{p}-\mathbf{p}^{\prime},
$$

where $\Delta(\mathbf{p})=\delta_{\mathbf{p} 0}$, and

$$
\begin{gathered}
g_{\alpha \beta}(\mathbf{k})=\int d \mathbf{y}\left(e_{1} e^{i \mathbf{k y}\left(m_{2} / M\right)}+e_{2} e^{-i \mathbf{k y}\left(m_{1} / M\right)}\right) \varphi_{\alpha}^{*}(\mathbf{y}) \varphi_{\beta}(\mathbf{y}) \\
\left.\mathbf{g}_{\alpha \beta}(\mathbf{k})=-\frac{i}{2} \int d \mathbf{y}\left(\frac{e_{1}}{m_{1}} e^{i \mathbf{k y}\left(m_{2} / M\right)}-\frac{e_{2}}{m_{2}} e^{-i \mathbf{k y}\left(m_{1} / M\right.}\right)\right)\left(\varphi_{\alpha}^{*}(\mathbf{y}) \frac{\partial \varphi_{\beta}(\mathbf{y})}{\partial \mathbf{y}}-\frac{\partial \varphi_{\alpha}^{*}(\mathbf{y})}{\partial \mathbf{y}} \varphi_{\beta}(\mathbf{y})\right) .
\end{gathered}
$$

The matrix element of $\hat{S}$-matrix is defined, in accordance with (67), by

$$
\langle f|\hat{S}| i\rangle=-2 \pi i\langle f|\tilde{\hat{V}}| i\rangle \delta\left(\varepsilon(\mathbf{p})-\varepsilon\left(\mathbf{p}^{\prime}\right)-\omega_{k}\right) .
$$

Next using the standard method we obtain the following expression for the probability of transition per unit time from the initial state $\hat{\eta}_{\alpha \mathbf{p}}^{+}|0\rangle$ into the final state $\hat{\eta}_{\beta \mathbf{p}^{\prime}}^{+} \hat{C}_{\mathbf{k} \lambda}^{+}|0\rangle$ :

$$
d w_{i \rightarrow f}=\frac{2 \pi}{\omega_{k}}\left|\mathbf{e}_{\mathbf{k}}^{(\lambda)}\left(\mathbf{g}_{\alpha \beta}(\mathbf{k})+\frac{\mathbf{p}+\mathbf{p}^{\prime}}{2 M} g_{\alpha \beta}(\mathbf{k})\right)\right|^{2} \delta\left(\omega_{k}+\varepsilon_{\beta}\left(\mathbf{p}^{\prime}\right)-\varepsilon_{\alpha}(\mathbf{p})\right) d \mathbf{k} .
$$

In the domain of small $\mathbf{k}\left(k r_{0}<<1\right.$, see section II) the Fourier-components of $\mathbf{g}_{\alpha \beta}(\mathbf{x}), g_{\alpha \beta}(\mathbf{x})$ can be written in the form

$$
\mathbf{g}_{\alpha \beta}(0)=\left(\frac{e_{1}}{m_{1}}-\frac{e_{2}}{m_{2}}\right) \int d \mathbf{y} \varphi_{\alpha}^{*}(\mathbf{y}) \hat{\mathbf{p}} \varphi_{\beta}(\mathbf{y})=\left(\frac{e_{1}}{m_{1}}-\frac{e_{2}}{m_{2}}\right)\langle\alpha|\hat{\mathbf{p}}| \beta\rangle, \quad g_{\alpha \beta}(0)=\left(e_{1}+e_{2}\right) \delta_{\alpha \beta} .
$$

If $e_{2}=-e_{1}=-e$ (this case corresponds to the hydrogen atom), then $g_{\alpha \beta}(0)=0, \mathbf{g}_{\alpha \beta}(0)=(e / \mu)\langle\alpha|\hat{\mathbf{p}}| \beta\rangle$. The Schroedinger equation for the wave function $\varphi_{\alpha}(\mathbf{y})$ gives

$$
\langle\alpha|\hat{\mathbf{p}}| \beta\rangle=i \mu\left(\varepsilon_{\alpha}-\varepsilon_{\beta}\right) \int d \mathbf{y} \varphi_{\alpha}^{*}(\mathbf{y}) \mathbf{y} \varphi_{\beta}(\mathbf{y}) .
$$

Therefore,

$$
\mathbf{g}_{\alpha \beta}(0)=i\left(\varepsilon_{\alpha}-\varepsilon_{\beta}\right)\langle\alpha|\hat{\mathbf{d}}| \beta\rangle,
$$

where $\hat{\mathbf{d}}=e \hat{\mathbf{y}}$ is the dipole moment of the atom. Hence,

$$
d w_{i \rightarrow f}=2 \pi \omega_{k}\left|\left\langle\alpha\left|\mathbf{e}_{\mathbf{k}}^{(\lambda)} \hat{\mathbf{d}}\right| \beta\right\rangle\right|^{2} \delta\left(\omega_{k}+\varepsilon_{\beta}\left(\mathbf{p}^{\prime}\right)-\varepsilon_{\alpha}(\mathbf{p})\right) d \mathbf{k} .
$$


Since $\varepsilon_{\alpha}(\mathbf{p})=\varepsilon_{\alpha}+p^{2} / 2 M$, we have for the atom of infinite mass $\left(m_{2} \rightarrow \infty, M \rightarrow \infty\right)^{5}$ :

$$
d w_{i \rightarrow f}=\frac{2 \pi}{\omega_{k}}\left|\mathbf{e}_{\mathbf{k}}^{(\lambda)} \mathbf{g}_{\alpha \beta}(\mathbf{k})\right|^{2} \delta\left(\omega_{k}+\varepsilon_{\beta}-\varepsilon_{\alpha}\right) d \mathbf{k},
$$

where

$$
\mathbf{g}_{\alpha \beta}(\mathbf{k})=-\frac{i e}{2 m_{1}} \int d \mathbf{y} e^{i \mathbf{k y}}\left(\varphi_{\alpha}^{*}(\mathbf{y}) \frac{\partial \varphi_{\beta}(\mathbf{y})}{\partial \mathbf{y}}-\frac{\partial \varphi_{\alpha}^{*}(\mathbf{y})}{\partial \mathbf{y}} \varphi_{\beta}(\mathbf{y})\right)
$$

( $m_{1}$ is the mass of light fermion).

\section{SCATTERING OF PHOTONS AND FERMIONS BY ATOMS}

Here we study the scattering process of long-wave photons by atoms. For this purpose let us use the general formulas (67), (68), where the Hamiltonian of free particles is defined by

$$
\tilde{\hat{\mathcal{H}}}_{0}=\sum_{i=1}^{2} \int d \mathbf{x} \nabla \hat{\chi}_{i}^{+}(\mathbf{x}) \nabla \hat{\chi}_{i}(\mathbf{x})+\sum_{\mathbf{k}} \sum_{\lambda=1}^{2} \omega_{k} \hat{C}_{\mathbf{k} \lambda}^{+} \hat{C}_{\mathbf{k} \lambda}+\sum_{\alpha} \int d \mathbf{x}\left(\frac{1}{2 m} \nabla \hat{\eta}_{\alpha}^{+}(\mathbf{x}) \nabla \hat{\eta}_{\alpha}(\mathbf{x})+\varepsilon_{\alpha} \hat{\eta}_{\alpha}^{+}(\mathbf{x}) \hat{\eta}_{\alpha}(\mathbf{x})\right),
$$

and the Hamiltonian associated with electromagnetic interaction by

$$
\tilde{\hat{V}}=\tilde{\hat{V}}^{\prime}+\tilde{\hat{V}}^{\prime \prime}
$$

In accordance with (48), (50),

$$
\begin{gathered}
\tilde{\hat{V}}^{\prime}=\frac{1}{2} \int d \mathbf{x} \hat{\mathbf{a}}^{2}(\mathbf{x})\left(\frac{e^{2}}{m_{1}} \tilde{\hat{\rho}}_{1}(\mathbf{x})+\frac{e^{2}}{m_{2}} \tilde{\hat{\hat{\rho}}}_{2}(\mathbf{x})\right), \quad \tilde{\hat{V}}^{\prime \prime}=-\int d \mathbf{x} \hat{\mathbf{a}}(\mathbf{x}) \tilde{\hat{\mathbf{j}}}(\mathbf{x}), \\
\hat{\hat{\mathbf{j}}}(\mathbf{x})=\frac{e}{m_{1}} \tilde{\hat{\boldsymbol{\pi}}}_{1}(\mathbf{x})+\frac{e}{m_{2}} \tilde{\hat{\pi}}_{2}(\mathbf{x})
\end{gathered}
$$

(further we assume $e_{1}=-e_{2}=e$ ). Since $\tilde{\hat{V}}^{\prime} \sim e^{2}, \tilde{\hat{V}}^{\prime \prime} \sim e$, in the second order in charge $e$, the $T$-matrix can be written as

$$
\hat{T}(z)=\tilde{\hat{V}}^{\prime}+\tilde{\hat{V}}^{\prime \prime} \hat{R}_{0}(z) \tilde{\hat{V}}^{\prime \prime}, \quad \hat{R}_{0}=\frac{1}{z-\tilde{\hat{\mathcal{H}}}_{0}}
$$

(within the considered approximation it is not necessary to take into account the fields $\hat{\chi}_{i}(\mathbf{x})$ ). Bearing in mind (72), (28), the operator $\tilde{\hat{V}}^{\prime}$ can be written as

$$
\tilde{\hat{V}}^{\prime}=\frac{1}{2 \mathcal{V}} \sum_{\mathbf{p}_{1}, \mathbf{p}_{2}} \hat{\eta}_{\alpha}^{+}\left(\mathbf{p}_{1}\right) \hat{\eta}_{\beta}\left(\mathbf{p}_{2}\right) q_{\alpha \beta}\left(\mathbf{p}_{1}-\mathbf{p}_{2}\right) \int d \mathbf{x} \hat{\mathbf{a}}^{2}(\mathbf{x}) e^{i \mathbf{x}\left(\mathbf{p}_{2}-\mathbf{p}_{1}\right)}
$$

where

$$
q_{\alpha \beta}(\mathbf{k})=\int d \mathbf{y}\left\{\frac{e^{2}}{m_{1}} e^{i \mathbf{k y}\left(m_{2} / M\right)}+\frac{e^{2}}{m_{2}} e^{-i \mathbf{k y}\left(m_{1} / M\right)}\right\} \varphi_{\alpha}^{*}(\mathbf{y}) \varphi_{\beta}(\mathbf{y})
$$

Similarly,

$$
\tilde{\hat{V}}^{\prime \prime}=-\frac{1}{\mathcal{V}} \sum_{\mathbf{p}_{1}, \mathbf{p}_{2}} \hat{\eta}_{\alpha}^{+}\left(\mathbf{p}_{1}\right) \hat{\eta}_{\beta}\left(\mathbf{p}_{2}\right) \mathbf{I}_{\alpha \beta}\left(\mathbf{p}_{1}+\mathbf{p}_{2}, \mathbf{p}_{1}-\mathbf{p}_{2}\right) \int d \mathbf{x} \hat{\mathbf{a}}(\mathbf{x}) e^{i \mathbf{x}\left(\mathbf{p}_{2}-\mathbf{p}_{1}\right)}
$$


where

$$
\mathbf{I}_{\alpha \beta}\left(\mathbf{p}_{1}+\mathbf{p}_{2}, \mathbf{p}_{1}-\mathbf{p}_{2}\right)=\mathbf{g}_{\alpha \beta}\left(\mathbf{p}_{1}-\mathbf{p}_{2}\right)+\frac{\mathbf{p}_{1}+\mathbf{p}_{2}}{2 M} g_{\alpha \beta}\left(\mathbf{p}_{1}-\mathbf{p}_{2}\right), \quad \mathbf{p}_{1}-\mathbf{p}_{2}=\mathbf{k}, \quad \mathbf{p}_{1}+\mathbf{p}_{2}=\mathbf{p}
$$

moreover, $\mathbf{g}_{\alpha \beta}(\mathbf{k}), g_{\alpha \beta}(\mathbf{k})$ are defined by (170).

Let $\hat{C}_{\mathbf{k} \lambda}^{+} \hat{\eta}_{\alpha}^{+}(\mathbf{p})|0\rangle$ and $\hat{C}_{\mathbf{k}^{\prime} \lambda^{\prime}}^{+} \hat{\eta}_{\alpha^{\prime}}^{+}\left(\mathbf{p}^{\prime}\right)|0\rangle$ be the initial and final states of the system,

$$
|i\rangle=\hat{C}_{\mathbf{k} \lambda}^{+} \hat{\eta}_{\alpha}^{+}(\mathbf{p})|0\rangle, \quad|i\rangle=\hat{C}_{\mathbf{k}^{\prime} \lambda^{\prime}}^{+} \hat{\eta}_{\alpha^{\prime}}^{+}\left(\mathbf{p}^{\prime}\right)|0\rangle .
$$

Then the matrix element $\langle f|\hat{T}(z)| i\rangle$ can be represented in the form

$$
\langle f|\hat{T}(z)| i\rangle=\left\langle f\left|\tilde{\hat{V}}^{\prime}\right| i\right\rangle+\left\langle f\left|\tilde{\hat{V}}^{\prime \prime} \hat{R}_{0}(z) \tilde{\hat{V}}^{\prime \prime}\right| i\right\rangle
$$

where

$$
\begin{gathered}
\left\langle f\left|\tilde{\hat{V}}^{\prime}\right| i\right\rangle=-\frac{4 \pi^{2} i}{\mathcal{V}} \frac{1}{\sqrt{\omega \omega^{\prime}}} R_{\alpha^{\prime} \alpha}^{\prime} \Delta\left(\mathbf{p}+\mathbf{k}-\mathbf{p}^{\prime}-\mathbf{k}^{\prime}\right) \\
\left\langle f\left|\tilde{\hat{V}}^{\prime \prime} \hat{R}_{0}(z) \tilde{\hat{V}}^{\prime \prime}\right| i\right\rangle=-\frac{4 \pi^{2} i}{\mathcal{V}} \frac{1}{\sqrt{\omega \omega^{\prime}}} R_{\alpha^{\prime} \alpha}^{\prime \prime} \Delta\left(\mathbf{p}+\mathbf{k}-\mathbf{p}^{\prime}-\mathbf{k}^{\prime}\right), \quad \Delta(\mathbf{k})=\delta_{\mathbf{k} 0},
\end{gathered}
$$

and the matrices $R_{\alpha^{\prime} \alpha}^{\prime}, R_{\alpha^{\prime} \alpha}^{\prime \prime}$ are given by

$$
\begin{gathered}
R_{\alpha^{\prime} \alpha}^{\prime}=\mathbf{e}_{\mathbf{k}}^{(\lambda)} \mathbf{e}_{\mathbf{k}}^{(\lambda)^{*}} q_{\alpha \alpha^{\prime}}(\mathbf{k}), \quad \mathbf{k}=\mathbf{p}^{\prime}-\mathbf{p} \\
R_{\alpha^{\prime} \alpha}^{\prime \prime}=\sum_{\beta}\left\{\frac{\left(\mathbf{e}^{\prime} \mathbf{I}_{\beta \alpha^{\prime}}\left(\mathbf{p}^{\prime}, \mathbf{k}^{\prime}\right)\right)^{*}\left(\mathbf{e} \mathbf{I}_{\beta \alpha}(\mathbf{p}, \mathbf{k})\right)}{\omega+\varepsilon_{\alpha}(\mathbf{p})-\varepsilon_{\beta}(\mathbf{p}+\mathbf{k})}+\frac{\left(\mathbf{e}^{\prime} \mathbf{I}_{\alpha \beta}\left(\mathbf{p},-\mathbf{k}^{\prime}\right)\right)^{*}\left(\mathbf{e I}_{\alpha^{\prime} \beta}\left(\mathbf{p}^{\prime},-\mathbf{k}\right)\right)}{-\omega^{\prime}+\varepsilon_{\alpha}(\mathbf{p})-\varepsilon_{\beta}\left(\mathbf{p}-\mathbf{k}^{\prime}\right)}\right\} .
\end{gathered}
$$

Here we have taken into account that $\omega+\varepsilon_{\alpha}(\mathbf{p})=\omega^{\prime}+\varepsilon_{\beta}\left(\mathbf{p}^{\prime}\right)$ is a consequence of (67) and assumed $E=\omega+\varepsilon_{\alpha}(\mathbf{p})$. According to our approximation $k r_{0}<<1\left(\lambda>>r_{0}\right)$ we have

$$
\begin{gathered}
q_{\alpha \beta}(\mathbf{k}) \approx \frac{e^{2}}{\mu} \delta_{\alpha \beta}, \quad g_{\alpha \beta} \approx 0 \\
\mathbf{g}_{\alpha \beta}(\mathbf{k}) \approx-\frac{i e}{\mu} \int d \mathbf{y} \varphi_{\alpha}^{*}(\mathbf{y}) \frac{\partial \varphi_{\beta}(\mathbf{y})}{\partial \mathbf{y}}=\frac{e}{\mu}\langle\alpha|\hat{\mathbf{p}}| \beta\rangle
\end{gathered}
$$

whence

$$
R_{\alpha^{\prime} \alpha}^{\prime} \approx \frac{e^{2}}{\mu} \mathbf{e}_{\mathbf{k}}^{(\lambda)} \mathbf{e}_{\mathbf{k}^{\prime}}^{\left(\lambda^{\prime}\right)^{*}} \delta_{\alpha \alpha^{\prime}}, \quad \mathbf{I}_{\alpha \beta}(\mathbf{p}, \mathbf{k}) \approx \mathbf{g}_{\alpha \beta}(\mathbf{k}) \approx \frac{e}{\mu}\langle\alpha|\hat{\mathbf{p}}| \beta\rangle
$$

where $\hat{\mathbf{p}}=-i \nabla$ is the momentum operator. Noting that $\varepsilon_{\alpha}(\mathbf{p})=\varepsilon_{\alpha}+\left(p^{2} / 2 M\right)$ we neglect the kinetic energy in denominators of $R_{\alpha^{\prime} \alpha}^{\prime \prime}$ (the kinetic energy is small in comparison to binding energy of bound states; see section II). The result is

$$
R_{\alpha^{\prime} \alpha}^{\prime \prime}=\frac{e^{2}}{\mu^{2}} \sum_{\beta}\left\{\frac{\left\langle\alpha^{\prime}\left|\mathbf{e}^{\prime *} \hat{\mathbf{p}}\right| \beta\right\rangle\langle\beta|\mathbf{e} \hat{\mathbf{p}}| \alpha\rangle}{\omega+\varepsilon_{\alpha}-\varepsilon_{\beta}}+\frac{\left\langle\alpha^{\prime}|\mathbf{e} \hat{\mathbf{p}}| \beta\right\rangle\left\langle\beta\left|\mathbf{e}^{\prime *} \hat{\mathbf{p}}\right| \alpha\right\rangle}{-\omega^{\prime}+\varepsilon_{\alpha}-\varepsilon_{\beta}}\right\}
$$

Let us express this formula through the matrix elements of the dipole moment of atom. It is easy to verify (by using of (71) that

$$
\begin{aligned}
\frac{\left\langle\alpha^{\prime}\left|\mathbf{e}^{\prime *} \hat{\mathbf{p}}\right| \beta\right\rangle\langle\beta|\mathbf{e} \hat{\mathbf{p}}| \alpha\rangle}{\omega+\varepsilon_{\alpha}-\varepsilon_{\beta}} & =\mu^{2}\left(\varepsilon_{\alpha}-\varepsilon_{\beta}-\omega^{\prime}+\frac{\omega \omega^{\prime}}{\omega+\varepsilon_{\alpha}-\varepsilon_{\beta}}\right)\left\langle\alpha^{\prime}\left|\mathbf{e}^{\prime *} \hat{\mathbf{y}}\right| \beta\right\rangle\langle\beta|\mathbf{e} \hat{\mathbf{y}}| \alpha\rangle, \\
\frac{\left\langle\alpha^{\prime}|\mathbf{e} \hat{\mathbf{p}}| \beta\right\rangle\left\langle\beta\left|\mathbf{e}^{\prime *} \hat{\mathbf{p}}\right| \alpha\right\rangle}{-\omega^{\prime}+\varepsilon_{\alpha}-\varepsilon_{\beta}} & =\mu^{2}\left(\varepsilon_{\alpha^{\prime}}-\varepsilon_{\beta}+\omega^{\prime}+\frac{\omega \omega^{\prime}}{-\omega^{\prime}+\varepsilon_{\alpha}-\varepsilon_{\beta}}\right)\left\langle\alpha^{\prime}|\mathbf{e} \hat{\mathbf{y}}| \beta\right\rangle\left\langle\beta\left|\mathbf{e}^{\prime *} \hat{\mathbf{y}}\right| \alpha\right\rangle .
\end{aligned}
$$


Therefore, the quantity $R_{\alpha^{\prime} \alpha}^{\prime \prime}$ is of the form

$$
R_{\alpha^{\prime} \alpha}^{\prime \prime}=Q_{\alpha^{\prime} \alpha}+\omega \omega^{\prime} \sum_{\beta}\left\{\frac{\left\langle\alpha^{\prime}|\mathbf{e} \hat{\mathbf{d}}| \beta\right\rangle\left\langle\beta\left|\mathbf{e}^{\prime *} \hat{\mathbf{d}}\right| \alpha\right\rangle}{-\omega^{\prime}+\varepsilon_{\alpha}-\varepsilon_{\beta}}+\frac{\left\langle\alpha^{\prime}\left|\mathbf{e}^{\prime *} \hat{\mathbf{d}}\right| \beta\right\rangle\langle\beta|\mathbf{e} \hat{\mathbf{d}}| \alpha\rangle}{\omega+\varepsilon_{\alpha}-\varepsilon_{\beta}}\right\},
$$

where $\hat{\mathbf{d}}=e \hat{\mathbf{y}}$ is the dipole moment of atom, and

$$
Q_{\alpha^{\prime} \alpha}=\mu^{2} \sum_{\beta}\left\{\left(\varepsilon_{\alpha}-\varepsilon_{\beta}-\omega^{\prime}\right)\left\langle\alpha^{\prime}\left|\mathbf{e}^{\prime *} \hat{\mathbf{y}}\right| \beta\right\rangle\langle\beta|\mathbf{e} \hat{\mathbf{y}}| \alpha\rangle+\left(\omega^{\prime}+\varepsilon_{\alpha^{\prime}}-\varepsilon_{\beta}\right)\left\langle\alpha^{\prime}|\mathbf{e} \hat{\mathbf{y}}| \beta\right\rangle\left\langle\beta\left|\mathbf{e}^{\prime *} \hat{\mathbf{y}}\right| \alpha\right\rangle\right\} .
$$

Next, using that

$$
\left[\hat{\mathbf{y}} \mathbf{e}, \frac{p^{2}}{2 \mu}+\hat{V}\right]=\left[\hat{\mathbf{y}} \mathbf{e}, \frac{p^{2}}{2 \mu}\right]=\frac{i}{\mu} \mathbf{e} \hat{\mathbf{p}}
$$

one finds

$$
Q_{\alpha^{\prime} \alpha}=-\frac{e^{2}}{\mu} \mathbf{e}^{\prime *} \mathbf{e} \delta_{\alpha \alpha^{\prime}}
$$

According to (73), the quantity $R_{\alpha^{\prime} \alpha}^{\prime}$ is equal to $R_{\alpha^{\prime} \alpha}^{\prime}=\frac{e^{2}}{\mu} \mathbf{e}^{\prime *} \mathbf{e} \delta_{\alpha \alpha^{\prime}}$. Thus, the matrix $R=R^{\prime}+R^{\prime \prime}$ takes the form

$$
R_{\alpha^{\prime} \alpha}=\omega \omega^{\prime} \sum_{\beta}\left\{\frac{\left\langle\alpha^{\prime}|\mathbf{e} \hat{\mathbf{d}}| \beta\right\rangle\left\langle\beta\left|\mathbf{e}^{\prime *} \hat{\mathbf{d}}\right| \alpha\right\rangle}{-\omega^{\prime}+\varepsilon_{\alpha}-\varepsilon_{\beta}}+\frac{\left\langle\alpha^{\prime}\left|\mathbf{e}^{\prime *} \hat{\mathbf{d}}\right| \beta\right\rangle\langle\beta|\mathbf{e} \hat{\mathbf{d}}| \alpha\rangle}{\omega+\varepsilon_{\alpha}-\varepsilon_{\beta}}\right\}
$$

and the amplitude of transition from the initial state into the final state is given by $\left(\mathrm{see}^{5}\right)$

$$
S_{i \rightarrow f}=-\frac{4 \pi^{2} i}{\mathcal{V}} \frac{1}{\sqrt{\omega \omega^{\prime}}} R_{\alpha^{\prime} \alpha} \delta\left(\varepsilon_{\alpha}(\mathbf{p})+\omega-\varepsilon_{\alpha^{\prime}}\left(\mathbf{p}^{\prime}\right)-\omega^{\prime}\right) \Delta\left(\mathbf{p}+\mathbf{k}-\mathbf{p}^{\prime}-\mathbf{k}^{\prime}\right) .
$$

The differential scattering cross-section is of the form

$$
d \sigma_{i \rightarrow f}=\frac{1}{\omega \omega^{\prime}}\left|R_{\alpha^{\prime} \alpha}\right|^{2} \delta\left(\varepsilon_{\alpha}(\mathbf{p})+\omega-\varepsilon_{\alpha^{\prime}}\left(\mathbf{p}^{\prime}\right)-\omega^{\prime}\right) d \mathbf{k}^{\prime} .
$$

Let us consider the scattering of an elementary particle by compound particle (the scattering of an electron by atom). Due to the structure of the Hamiltonian of interaction (38) it is sufficient to study the scattering of particle of the first kind by bound state. The matrix element of $\hat{S}$-matrix in the first nonvanishing approximation over interaction is defined by

$$
S_{i \rightarrow f}=-2 \pi i\left\langle f\left|\tilde{\hat{\mathcal{H}}}_{i n t}^{1}\right| i\right\rangle \delta\left(\varepsilon_{i}-\varepsilon_{f}\right),
$$

where $\tilde{\hat{\mathcal{H}}}_{\text {int }}^{1}$ is given by (38) and consequently,

$$
\left\langle f\left|\tilde{\hat{\mathcal{H}}}_{i n t}^{1}\right| i\right\rangle=\frac{1}{\mathcal{V}^{2}} \int d \mathbf{x}_{1} d \mathbf{x}_{2} d \mathbf{y} \varphi_{\alpha}\left(\mathbf{x}_{2}-\mathbf{y}\right) e^{i \mathbf{p} \mathbf{X}} \varphi_{\alpha^{\prime}}\left(\mathbf{x}_{2}-\mathbf{y}\right) e^{-i \mathbf{p}^{\prime} \mathbf{X}}\left(\nu_{11}\left(\mathbf{x}_{1}-\mathbf{x}_{2}\right)-\nu_{21}\left(\mathbf{x}_{1}-\mathbf{y}\right)\right) e^{i \mathbf{x}_{1}\left(\mathbf{k}_{1}-\mathbf{k}_{1}^{\prime}\right)} .
$$

Here $\langle f|=\langle 0| \hat{\eta}_{\alpha^{\prime}}\left(\mathbf{p}^{\prime}\right) \hat{\chi}_{1}\left(\mathbf{k}_{1}^{\prime}\right)$ and $|i\rangle=\hat{\chi}_{1}^{+}\left(\mathbf{k}_{1}\right) \hat{\eta}_{\alpha}^{+}(\mathbf{p})|0\rangle$. The simple calculations lead to

$$
\left\langle f\left|\tilde{\hat{\mathcal{H}}}_{i n t}^{1}\right| i\right\rangle=\frac{1}{\mathcal{V}} \Delta\left(\mathbf{p}+\mathbf{k}-\mathbf{p}^{\prime}-\mathbf{k}^{\prime}\right) \int d \mathbf{x} \varphi_{\alpha^{\prime}}^{*}(\mathbf{x}) \varphi_{\alpha}(\mathbf{x})\left\{\nu_{11}(\mathbf{q}) e^{-i \mathbf{q} \mathbf{x}\left(m_{2} / M\right)}+\nu_{21}(\mathbf{q}) e^{i \mathbf{q} \mathbf{x}\left(m_{1} / M\right)}\right\}, \quad \mathbf{q}=\mathbf{k}^{\prime}-\mathbf{k} .
$$

For Coulomb's interaction of particles we have

$$
\nu_{11}(\mathbf{q})=-\nu_{21}(\mathbf{q}) \equiv \nu(\mathbf{q})=\frac{4 \pi e^{2}}{q^{2}} .
$$


Therefore, in the long-wave approximation $\left(q r_{0}<<1\right)$, one gets

$$
\left\langle f\left|\tilde{\hat{\mathcal{H}}}_{i n t}^{1}\right| i\right\rangle=4 \pi i e \frac{1}{\mathcal{V}} \Delta\left(\mathbf{p}+\mathbf{k}-\mathbf{p}^{\prime}-\mathbf{k}^{\prime}\right) \frac{1}{q^{2}} \mathbf{q d}_{\alpha^{\prime} \alpha}
$$

where $\hat{\mathbf{d}}=e \hat{\mathbf{x}}$ is the dipole moment of atom $\left(\mathbf{d}_{\alpha^{\prime} \alpha}=\int d \mathbf{x} \varphi_{\alpha^{\prime}}^{*} \mathbf{x}(\mathbf{x}) \varphi_{\alpha}(\mathbf{x})\right)$. Note that the Hamiltonian

$$
\tilde{\hat{V}}=\int d \mathbf{x}_{1} d \mathbf{x}_{2} \hat{\chi}^{+}\left(\mathbf{x}_{1}\right) \hat{\chi}\left(\mathbf{x}_{1}\right) v_{\alpha^{\prime} \alpha}\left(\mathbf{x}_{1}-\mathbf{x}_{2}\right) \hat{\eta}_{\alpha^{\prime}}^{+}\left(\mathbf{x}_{2}\right) \hat{\eta}_{\alpha}\left(\mathbf{x}_{2}\right),
$$

which describes the interaction between particles and bound states leads to the same result. Here $\hat{\chi}, \hat{\eta}_{\alpha}$ are the field operators of particles and bound states respectively, and $v_{\alpha^{\prime} \alpha}(\mathbf{x})=\left(e / x^{3}\right) \mathbf{x d}_{\alpha^{\prime} \alpha}$. The relation (74) defines the interaction energy of the dipole moment $\mathbf{d}$ and electric charge $e$, which are at the distance $\mathbf{x}$ from each other.

\section{THE VAN DER WAALS FORCES}

In this section we investigate the forces acting between neutral atoms being in the ground state (the van der Waals forces) on the basis of the developed formalism. In order to solve this problem let us address to the Schroedinger equation that determines the energy spectrum of the system,

$$
\tilde{\hat{\mathcal{H}}} \Phi=E \Phi, \quad \tilde{\hat{\mathcal{H}}}=\tilde{\hat{\mathcal{H}}}_{0}+\tilde{\hat{V}} .
$$

Here $\tilde{\hat{\mathcal{H}}}_{0}$ and $\tilde{\hat{V}}=\tilde{\hat{\mathcal{H}}}_{\text {int }}^{1}+\tilde{\hat{\mathcal{H}}}_{\text {int }}^{2}+\tilde{\hat{\mathcal{H}}}_{\text {int }}^{3}$ are defined by (37), [38)- (40). Since the system in question consists of two atoms, we should seek the solution of (75) in the form

$$
\Phi_{\alpha \beta}\left(\mathbf{X}, \mathbf{X}^{\prime}\right)=\sum_{\lambda \rho} \int d \mathbf{Y} d \mathbf{Y}^{\prime} K_{\alpha \beta ; \lambda \rho}\left(\mathbf{X}, \mathbf{X}^{\prime} ; \mathbf{Y}, \mathbf{Y}^{\prime}\right) \hat{\eta}_{\lambda}^{+}(\mathbf{Y}) \hat{\eta}_{\rho}^{+}\left(\mathbf{Y}^{\prime}\right) \Phi_{0}
$$

The Hamiltonian of interaction $\tilde{\hat{V}}$ is equal, in accordance with (38)- to

$$
\tilde{\hat{V}}=\frac{1}{2} \int d \mathbf{X} d \mathbf{Y} \hat{\eta}_{\alpha}^{+}(\mathbf{X}) \hat{\eta}_{\beta}^{+}(\mathbf{Y}) \hat{\eta}_{\gamma}(\mathbf{Y}) \hat{\eta}_{\delta}(\mathbf{X}) G_{\delta \gamma ; \alpha \beta}(\mathbf{X}-\mathbf{Y})
$$

where

$$
\begin{aligned}
G_{\delta \gamma ; \alpha \beta}(\mathbf{X}-\mathbf{Y})=\int d \mathbf{x} d \mathbf{y} \varphi_{\alpha}^{*}(\mathbf{x}) \varphi_{\beta}^{*}(\mathbf{y}) \varphi_{\gamma}(\mathbf{y}) \varphi_{\delta}(\mathbf{x})\{ & \nu_{12}\left(\mathbf{X}-\mathbf{Y}-\frac{m_{1} \mathbf{x}+m_{2} \mathbf{y}}{M}\right)+\nu_{21}\left(\mathbf{X}-\mathbf{Y}+\frac{m_{1} \mathbf{y}+m_{2} \mathbf{x}}{M}\right) \\
& \left.+\nu_{11}\left(\mathbf{X}-\mathbf{Y}+\frac{m_{2}}{M}(\mathbf{x}-\mathbf{y})\right)+\nu_{22}\left(\mathbf{X}-\mathbf{Y}-\frac{m_{1}}{M}(\mathbf{x}-\mathbf{y})\right)\right\} .
\end{aligned}
$$

We suppose that the kinetic energy of atoms is small in comparison to the energy levels $\left|\varepsilon_{\alpha}\right|\left(\varepsilon_{\alpha}<0\right)$. In this case, according to (37), the operator $\tilde{\hat{\mathcal{H}}}_{0}$ can be represented in the form

$$
\tilde{\hat{\mathcal{H}}}_{0}=\sum_{\alpha} \int d \mathbf{X} \varepsilon_{\alpha} \hat{\eta}_{\alpha}^{+}(\mathbf{X}) \hat{\eta}_{\alpha}(\mathbf{X})
$$

It can be easily seen that

$$
\begin{gathered}
\tilde{\hat{\mathcal{H}}}_{0} \hat{\eta}_{\lambda}^{+}(\mathbf{Z}) \hat{\eta}_{\rho}^{+}\left(\mathbf{Z}^{\prime}\right) \Phi_{0}=\left(\varepsilon_{\lambda}+\varepsilon_{\rho}\right) \hat{\eta}_{\lambda}^{+}(\mathbf{Z}) \hat{\eta}_{\rho}^{+}\left(\mathbf{Z}^{\prime}\right) \Phi_{0}, \\
\tilde{\hat{V}} \hat{\eta}_{\lambda}^{+}(\mathbf{Z}) \hat{\eta}_{\rho}^{+}\left(\mathbf{Z}^{\prime}\right) \Phi_{0}=\sum_{\alpha \beta} G_{\lambda \rho ; \alpha \beta}\left(\mathbf{Z}-\mathbf{Z}^{\prime}\right) \hat{\eta}_{\alpha}^{+}(\mathbf{Z}) \hat{\eta}_{\beta}^{+}\left(\mathbf{Z}^{\prime}\right) \Phi_{0} .
\end{gathered}
$$


These formulas show that we can seek the solution of (75) in a more simple, rather than (76), form

$$
\Phi_{\alpha \beta}\left(\mathbf{X}, \mathbf{X}^{\prime}\right)=\sum_{\lambda \rho} K_{\alpha \beta ; \lambda \rho}\left(\mathbf{X}, \mathbf{X}^{\prime}\right) \hat{\eta}_{\lambda}^{+}(\mathbf{X}) \hat{\eta}_{\rho}^{+}\left(\mathbf{X}^{\prime}\right) \Phi_{0}
$$

so that the coordinates of atoms have the definite values in the state $\Phi_{\alpha \beta}\left(\mathbf{X}, \mathbf{X}^{\prime}\right)$. Upon substituting this expression into (75) and using (78), one finds

$$
K_{\alpha \beta ; \gamma \delta}\left(\varepsilon_{\gamma}+\varepsilon_{\delta}\right)+\sum_{\lambda \rho} K_{\alpha \beta ; \lambda \rho} G_{\lambda \rho ; \gamma \delta}\left(\mathbf{Z}-\mathbf{Z}^{\prime}\right)=E_{\alpha \beta} K_{\alpha \beta ; \gamma \delta} .
$$

A perturbative approach for this equation can be easily developed in the domain of great $\left|\mathbf{Z}-\mathbf{Z}^{\prime}\right|$ when the quantity $G_{\lambda \rho ; \gamma \delta}\left(\mathbf{Z}-\mathbf{Z}^{\prime}\right)$ becomes small (see (77)). Expanding $K_{\alpha \beta ; \gamma \delta}$ in $G$,

$$
\begin{gathered}
K_{\alpha \beta ; \gamma \delta}=K_{\alpha \beta ; \gamma \delta}^{1}+K_{\alpha \beta ; \gamma \delta}^{1}+K_{\alpha \beta ; \gamma \delta}^{2}+\ldots, \\
E_{\alpha \beta}=E_{\alpha \beta}^{0}+E_{\alpha \beta}^{1}+E_{\alpha \beta}^{2}+\ldots
\end{gathered}
$$

one obtains in the zeroth order

$$
K_{\alpha \beta ; \gamma \delta}^{0}\left(\varepsilon_{\gamma}+\varepsilon_{\delta}\right)=E_{\alpha \beta}^{0} K_{\alpha \beta ; \gamma \delta}^{0}
$$

whence

$$
K_{\alpha \beta ; \gamma \delta}^{0}=K_{\alpha \beta}^{0} \delta_{\alpha \gamma} \delta_{\beta \delta}, \quad E_{\alpha \beta}^{0}=\varepsilon_{\alpha}+\varepsilon_{\beta} .
$$

Taking into account this result we have in the first approximation,

$$
K_{\alpha \beta ; \gamma \delta}^{1}\left(\varepsilon_{\gamma}+\varepsilon_{\delta}\right)+K_{\alpha \beta}^{0} G_{\alpha \beta ; \gamma \delta}\left(\mathbf{Z}-\mathbf{Z}^{\prime}\right)=\left(\varepsilon_{\alpha}+\varepsilon_{\beta}\right) K_{\alpha \beta ; \gamma \delta}^{1}+E_{\alpha \beta}^{1} K_{\alpha \beta}^{0} \delta_{\alpha \gamma} \delta_{\beta \delta} .
$$

By setting here $\alpha=\gamma, \beta=\delta$, one gets

$$
E_{\alpha \beta}^{1}=G_{\alpha \beta ; \alpha \beta}\left(\mathbf{Z}-\mathbf{Z}^{\prime}\right)
$$

and for $\alpha, \beta \neq \gamma, \delta$,

$$
K_{\alpha \beta ; \gamma \delta}^{1}=K_{\alpha \beta}^{0} \frac{G_{\alpha \beta ; \gamma \delta}\left(\mathbf{Z}-\mathbf{Z}^{\prime}\right)}{\left(\varepsilon_{\alpha}+\varepsilon_{\beta}-\varepsilon_{\gamma}-\varepsilon_{\delta}\right)}, \quad \alpha, \beta \neq \gamma, \delta .
$$

The second order of the perturbative approach give

$$
K_{\alpha \beta ; \gamma \delta}^{2}\left(\varepsilon_{\gamma}+\varepsilon_{\delta}\right)+\sum_{\lambda \rho} K_{\alpha \beta ; \lambda \rho}^{1} G_{\lambda \rho ; \gamma \delta}\left(\mathbf{Z}-\mathbf{Z}^{\prime}\right)=\left(\varepsilon_{\alpha}+\varepsilon_{\beta}\right) K_{\alpha \beta ; \gamma \delta}^{2}+E_{\alpha \beta}^{1} K_{\alpha \beta ; \gamma \delta}^{1}+E_{\alpha \beta}^{2} K_{\alpha \beta ; \gamma \delta}^{0} .
$$

Taking here $\alpha=\gamma, \beta=\delta$, one finds

$$
\sum_{\lambda \rho} K_{\alpha \beta ; \lambda \rho}^{1} G_{\lambda \rho ; \alpha \beta}\left(\mathbf{Z}-\mathbf{Z}^{\prime}\right)=E_{\alpha \beta}^{1} K_{\alpha \beta ; \alpha \beta}+K_{\alpha \beta}^{0} E_{\alpha \beta}^{2},
$$

whence, according to (79), 800), we have

$$
E_{\alpha \beta}^{2}=\sum_{\lambda \rho}^{\prime} \frac{G_{\alpha \beta ; \lambda \rho}\left(\mathbf{Z}-\mathbf{Z}^{\prime}\right) G_{\lambda \rho ; \alpha \beta}\left(\mathbf{Z}-\mathbf{Z}^{\prime}\right)}{\left(\varepsilon_{\alpha}+\varepsilon_{\beta}-\varepsilon_{\lambda}-\varepsilon_{\rho}\right)} .
$$


The prime above the sum means that the terms with $\lambda=\alpha, \rho=\beta$ are omitted. The state vector $\Phi_{\alpha \beta}\left(\mathbf{X}, \mathbf{X}^{\prime}\right)$ in the main approximation of the perturbative approach is determined by

$$
\Phi_{\alpha \beta}\left(\mathbf{X}, \mathbf{X}^{\prime}\right)=K_{\alpha \beta}^{0} \hat{\eta}_{\alpha}^{+}(\mathbf{X}) \hat{\eta}_{\beta}^{+}\left(\mathbf{X}^{\prime}\right) \Phi_{0}+\ldots
$$

(the constant $K_{\alpha \beta}^{0}$ may be found from the normalization relation, $\left(\Phi_{\alpha \beta}, \Phi_{\alpha \beta}\right)=1$ ). Formulas (79), (81) for $E_{\alpha \beta}^{1}$ and $E_{\alpha \beta}^{2}$ give us the corrections to the energy levels $E_{\alpha \beta}^{0}=\varepsilon_{\alpha}+\varepsilon_{\beta}$. It follows from the obtained formulas that the energy of two atoms being in the ground state $\alpha$ and spaced apart for sufficiently long distances is defined by

$$
E_{\alpha \alpha}=2 \varepsilon_{\alpha}+G_{\alpha \alpha ; \alpha \alpha}\left(\mathbf{Z}-\mathbf{Z}^{\prime}\right)+\sum_{\lambda \rho}^{\prime} \frac{G_{\alpha \alpha ; \lambda \rho}\left(\mathbf{Z}-\mathbf{Z}^{\prime}\right) G_{\lambda \rho ; \alpha \alpha}\left(\mathbf{Z}-\mathbf{Z}^{\prime}\right)}{\left(2 \varepsilon_{\alpha}-\varepsilon_{\lambda}-\varepsilon_{\rho}\right)}+\ldots
$$

Let us prove now that $G_{\alpha \alpha ; \alpha \alpha}\left(\mathbf{Z}-\mathbf{Z}^{\prime}\right) \equiv 0$. In doing so we use the following formula ${ }^{9}$ :

$$
\frac{1}{\sqrt{R^{2}-2 R \rho x+\rho^{2}}}=\frac{1}{|\mathbf{R}-\boldsymbol{\rho}|}=\frac{1}{R}+\sum_{n=1}^{\infty}\left(\frac{\rho}{R}\right)^{n} \mathcal{P}_{n}(x)
$$

where $x=\cos \vartheta, \vartheta$ is the angle between vectors $\mathbf{R}$ and $\boldsymbol{\rho}$, and $\mathcal{P}_{n}(x)$ are the Legendre polynomials. Noting that (see (77))

$$
\begin{array}{r}
G_{\alpha \alpha ; \alpha \alpha}\left(\mathbf{Z}-\mathbf{Z}^{\prime}\right)=\int d \mathbf{x} d \mathbf{y}\left|\varphi_{\alpha}(\mathbf{x})\right|^{2}\left|\varphi_{\alpha}(\mathbf{y})\right|^{2}\left\{\nu_{12}\left(\mathbf{Z}-\mathbf{z}^{\prime}-\frac{m_{1} \mathbf{x}+m_{2} \mathbf{y}}{M}\right)+\nu_{21}\left(\mathbf{Z}-\mathbf{z}^{\prime}+\frac{m_{1} \mathbf{y}+m_{2} \mathbf{x}}{M}\right)\right. \\
\left.+\nu_{11}\left(\mathbf{Z}-\mathbf{z}^{\prime}+\frac{m_{2}}{M}(\mathbf{x}-\mathbf{y})\right)+\nu_{22}\left(\mathbf{Z}-\mathbf{z}^{\prime}-\frac{m_{1}}{M}(\mathbf{x}-\mathbf{y})\right)\right\}
\end{array}
$$

we come, taking into account the spherical symmetry of $\left|\varphi_{\alpha}(\mathbf{x})\right|^{2}$, to

$$
G_{\alpha \alpha ; \alpha \alpha}\left(\mathbf{Z}-\mathbf{Z}^{\prime}\right)=0
$$

We have also employed here that according to 43)

$$
\nu_{a b}\left(\mathbf{Z}-\mathbf{Z}^{\prime}\right)=\frac{e_{a} e_{b}}{\left|\mathbf{Z}-\mathbf{Z}^{\prime}\right|}, \quad e_{1}=-e_{2}=e .
$$

Let us find now $G_{\alpha \alpha ; \lambda \rho}\left(\mathbf{Z}-\mathbf{Z}^{\prime}\right)$,

$$
\begin{array}{r}
G_{\alpha \alpha ; \lambda \rho}\left(\mathbf{Z}-\mathbf{Z}^{\prime}\right)=\int d \mathbf{x} d \mathbf{y} \varphi_{\rho}^{*}(\mathbf{x}) \varphi_{\lambda}^{*}(\mathbf{y}) \varphi_{\alpha}(\mathbf{y}) \varphi_{\alpha}(\mathbf{x})\left\{\nu_{12}\left(\mathbf{Z}-\mathbf{z}^{\prime}-\frac{m_{1} \mathbf{x}+m_{2} \mathbf{y}}{M}\right)+\nu_{21}\left(\mathbf{Z}-\mathbf{z}^{\prime}+\frac{m_{1} \mathbf{y}+m_{2} \mathbf{x}}{M}\right)\right. \\
\left.+\nu_{11}\left(\mathbf{Z}-\mathbf{z}^{\prime}+\frac{m_{2}}{M}(\mathbf{x}-\mathbf{y})\right)+\nu_{22}\left(\mathbf{Z}-\mathbf{z}^{\prime}-\frac{m_{1}}{M}(\mathbf{x}-\mathbf{y})\right)\right\}
\end{array}
$$

The presence of multipliers $\varphi_{\alpha}(\mathbf{y})$ and $\varphi_{\alpha}(\mathbf{x})$ makes it possible to expand the expression in braces in powers of $\mathbf{x}, \mathbf{y}$. As a result we have

$$
G_{\alpha \alpha ; \beta \lambda}\left(\mathbf{Z}-\mathbf{Z}^{\prime}\right)=\frac{1}{\left|\mathbf{Z}-\mathbf{Z}^{\prime}\right|^{3}}\left(-3\left(\mathbf{n d} \mathbf{d}_{\beta \alpha}\right)\left(\mathbf{n d}_{\lambda \alpha}\right)+\left(\mathbf{d}_{\beta \alpha} \mathbf{d}_{\lambda \alpha}\right)\right)
$$

where

$$
\mathbf{n}=\frac{\mathbf{Z}-\mathbf{Z}^{\prime}}{\left|\mathbf{Z}-\mathbf{Z}^{\prime}\right|}, \quad \mathbf{d}_{\beta \alpha}=e \int d \mathbf{x} \mathbf{x} \varphi_{\beta}^{*}(\mathbf{x}) \varphi_{\alpha}(\mathbf{x})
$$


$\left(\mathbf{d}_{\beta \alpha}\right.$ are the matrix elements of the dipole moment of atom). Thus, the potential energy of interaction between atoms, $V\left(\mathbf{Z}-\mathbf{Z}^{\prime}\right)$ is defined by 6

$$
V\left(\mathbf{Z}-\mathbf{Z}^{\prime}\right) \equiv E_{\alpha \alpha}^{2}-2 \varepsilon_{\alpha}=\frac{1}{\left|\mathbf{Z}-\mathbf{Z}^{\prime}\right|^{6}} \sum_{\beta \lambda}^{\prime} \frac{\left|-3\left(\mathbf{n d} \mathbf{d}_{\beta \alpha}\right)\left(\mathbf{n d}_{\lambda \alpha}\right)+\left(\mathbf{d}_{\beta \alpha} \mathbf{d}_{\lambda \alpha}\right)\right|^{2}}{2 \varepsilon_{\alpha}-\varepsilon_{\beta}-\varepsilon_{\lambda}}<0 .
$$

Since $\varepsilon_{\beta}, \varepsilon_{\lambda}>\varepsilon_{\alpha}, V\left(\mathbf{Z}-\mathbf{Z}^{\prime}\right)<0$. Therefore, the attractive forces (the van der Waals forces) act between the neutral atoms at long distances.

Consider now the scattering of a compound particle by compound particle, which are in the ground state. We start from the scattering matrix representation in terms of $T$-operators (67). Accurate to the terms, which are of the second order in interaction $\tilde{\hat{V}}$ between the atoms (see (68) ), one gets

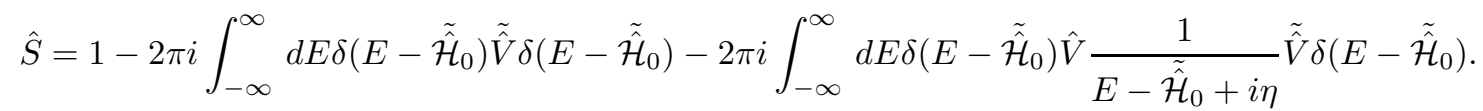

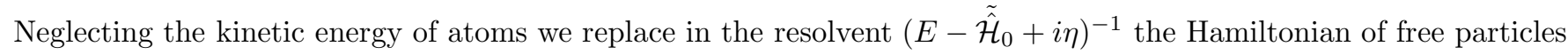
$\tilde{\hat{\mathcal{H}}}_{0}$ by the Hamiltonian $\tilde{\hat{H}}_{0}$ of atoms in the rest (see (37))

$$
\tilde{\hat{\mathcal{H}}}_{0} \rightarrow \tilde{\hat{H}}_{0}=\sum_{\alpha} \int d \mathbf{x} \varepsilon_{\alpha} \hat{\eta}_{\alpha}^{+}(\mathbf{x}) \hat{\eta}_{\alpha}(\mathbf{x})
$$

(in the low-energy limit we can not do this under the argument of $\delta$-function).

Since we study the collision of two atoms and the number of atoms is conserved during the process of collision, we can restrict ourselves by considering of this process in the two-particle subspace with the following completeness condition:

$$
\frac{1}{2} \int d \mathbf{x}_{1} d \mathbf{x}_{2} \sum_{\alpha_{1}, \alpha_{2}} \hat{\eta}_{\alpha_{1}}^{+}\left(\mathbf{x}_{1}\right) \hat{\eta}_{\alpha_{2}}^{+}\left(\mathbf{x}_{2}\right)|0\rangle\langle 0| \hat{\eta}_{\alpha_{2}}\left(\mathbf{x}_{2}\right) \hat{\eta}_{\alpha_{1}}\left(\mathbf{x}_{1}\right)=1
$$

In this case formulas (78) are true. Since the atoms are in the ground state, (83) is also valid and, therefore, the matrix element of $\hat{S}$-matrix turns to zero in the first approximation of the perturbation theory.

The second approximation of the perturbation theory is defined, according to (84), (85), by the formula

$$
\begin{aligned}
\tilde{\hat{V}} \frac{1}{E-\tilde{\hat{H}}_{0}+i \eta} \tilde{\hat{V}} & =\frac{1}{2} \sum_{\alpha_{1}, \alpha_{2}} \sum_{\beta_{1}, \beta_{2}} \sum_{\gamma_{1}, \gamma_{2}} \int d \mathbf{x}_{1} d \mathbf{x}_{2} \frac{1}{E-\varepsilon_{\alpha_{1}}-\varepsilon_{\alpha_{2}}+i \eta} G_{\alpha_{1} \alpha_{2} ; \beta_{1} \beta_{2}}\left(\mathbf{x}_{1}-\mathbf{x}_{2}\right) \\
& \times \hat{\eta}_{\beta_{1}}^{+}\left(\mathbf{x}_{1}\right) \hat{\eta}_{\beta_{2}}^{+}\left(\mathbf{x}_{2}\right)|0\rangle\langle 0| \hat{\eta}_{\gamma_{1}}\left(\mathbf{x}_{1}\right) \hat{\eta}_{\gamma_{2}}\left(\mathbf{x}_{2}\right) G_{\gamma_{1} \gamma_{2} ; \alpha_{1} \alpha_{2}}^{+}\left(\mathbf{x}_{1}-\mathbf{x}_{2}\right) .
\end{aligned}
$$

In the two-particle subspace this formula is equivalent to

$$
\tilde{\hat{V}} \frac{1}{E-\tilde{\hat{H}}_{0}+i \eta} \tilde{\hat{V}}=\int d \mathbf{x}_{1} d \mathbf{x}_{2} \sum_{\beta_{1}, \beta_{2}} \sum_{\gamma_{1}, \gamma_{2}} \hat{\eta}_{\beta_{1}}^{+}\left(\mathbf{x}_{1}\right) \hat{\eta}_{\beta_{2}}^{+}\left(\mathbf{x}_{2}\right) V_{\beta_{1} \beta_{2} ; \gamma_{1} \gamma_{2}}\left(\mathbf{x}_{1}-\mathbf{x}_{2} ; E\right) \hat{\eta}_{\gamma_{1}}\left(\mathbf{x}_{1}\right) \hat{\eta}_{\gamma_{2}}\left(\mathbf{x}_{2}\right),
$$

where

$$
V_{\beta_{1} \beta_{2} ; \gamma_{1} \gamma_{2}}\left(\mathbf{x}_{1}-\mathbf{x}_{2} ; E\right)=\sum_{\alpha_{1} \alpha_{2}} \frac{1}{E-\varepsilon_{\alpha_{1}}-\varepsilon_{\alpha_{2}}+i \eta} G_{\gamma_{1} \gamma_{2} ; \alpha_{1} \alpha_{2}}^{+}\left(\mathbf{x}_{1}-\mathbf{x}_{2}\right) G_{\alpha_{1} \alpha_{2} ; \beta_{1} \beta_{2}}\left(\mathbf{x}_{1}-\mathbf{x}_{2}\right) .
$$

Since the initial and final states of a particle are the ground states, the scattering matrix in the two-particle subspace can be represented in the form

$$
\hat{S}=-2 \pi i \int_{-\infty}^{\infty} d E \delta\left(E-\tilde{\hat{\mathcal{H}}}_{0}\right) \hat{V}_{e f f} \delta\left(E-\tilde{\hat{\mathcal{H}}}_{0}\right)
$$


where

$$
\begin{gathered}
\hat{V}_{e f f}=\frac{1}{2} \int d \mathbf{x}_{1} \int d \mathbf{x}_{2} \hat{\eta}_{\alpha_{1}}^{+}\left(\mathbf{x}_{1}\right) \hat{\eta}_{\alpha_{2}}^{+}\left(\mathbf{x}_{2}\right) V_{e f f}\left(\mathbf{x}_{1}-\mathbf{x}_{2}\right) \hat{\eta}_{\alpha_{2}}\left(\mathbf{x}_{2}\right) \hat{\eta}_{\alpha_{1}}\left(\mathbf{x}_{1}\right), \\
V_{e f f}\left(\mathbf{x}_{1}-\mathbf{x}_{2}\right)=\sum_{\beta_{1} \beta_{2}} \frac{G_{\alpha \alpha ; \beta_{1} \beta_{2}}^{+}\left(\mathbf{x}_{1}-\mathbf{x}_{2}\right) G_{\beta_{1} \beta_{2} ; \alpha \alpha}\left(\mathbf{x}_{1}-\mathbf{x}_{2}\right)}{2 \varepsilon_{\alpha}-\varepsilon_{\alpha_{1}}-\varepsilon_{\alpha_{2}}}
\end{gathered}
$$

We can see that the second order in $\tilde{\hat{V}}$ of perturbation theory for $\hat{S}$-matrix is equivalent to the first order of perturbation theory over effective interaction $\hat{V}_{\text {eff }}$. This effective interaction is determined by van der Waals forces (see formula (82)).

It follows from (39), that the Hamiltonian of interaction of atoms at low-energies is defined by

$$
\tilde{\hat{V}}=\int d \mathbf{x}_{1} d \mathbf{x}_{2} v_{\alpha \beta ; \gamma \delta}\left(\mathbf{x}_{1}-\mathbf{x}_{2}\right) \hat{\eta}_{\alpha}^{+}\left(\mathbf{x}_{1}\right) \hat{\eta}_{\beta}^{+}\left(\mathbf{x}_{2}\right) \hat{\eta}_{\gamma}\left(\mathbf{x}_{2}\right) \hat{\eta}_{\delta}\left(\mathbf{x}_{1}\right)
$$

where

$$
v_{\alpha \beta ; \gamma \delta}(\mathbf{x})=\frac{1}{x^{5}}\left(x^{2}\left(\mathbf{d}_{\alpha \delta} \mathbf{d}_{\beta \gamma}\right)-3\left(\mathbf{x d}_{\alpha \delta}\right)\left(\mathbf{x d}_{\delta \gamma}\right)\right) .
$$

This Hamiltonian corresponds to the dipole-dipole interaction of atoms.

\section{CONCLUSION}

The goal of this paper was to develop a microscopic approach for describing the physical processes in many-particle systems in the presence of bound states of particles. To achieve this goal we developed an approximate second quantization method for systems with bound states of particles.

The basic results obtained in this paper are the following:

1. The Fock space was introduced in the second quantization formalism. In this space the creation and annihilation operators of elementary particles $\hat{\chi}^{+}, \hat{\chi}$ and their bound states $\hat{\eta}^{+}, \hat{\eta}$ were introduced on an equal status.

2. The operators of basic physical quantities acting in this space were constructed. These operators include the Hamiltonians of interaction between elementary particles and their bound states.

3. It was shown that in the approximation when the radius of interaction is small the above mentioned Hamiltonians transform into the well-known Hamiltonians for Coulomb's and dipole interactions between the particles of various kinds.

4. The non-relativistic quantum electrodynamics of charged and neutral (the bound states) particles was constructed.

5. The various physical effects including the theory of the van der Waals forces acting between atoms were considered as the approbation of the developed formalism. The description of such effects within the usual formalism requires more considerable efforts associated with introduction of the interaction between neutral currents of bound states and electromagnetic field.

6. More detailed calculations, which fall out the limits of our approximation should result in appearance of three- and many-body interactions between unbound particles and two-body bound states. These many-body interactions should 
be consistent with pair interaction of original particles. The appearance of these many-body terms is analogous to the origin of the terms with photon-photon scattering in transition from the usual standard Lagrangian to the effective low-energy Euler-Heisenberg Lagrangian of quantum electrodynamics.

Especially we would like to emphasize a role of the obtained Hamiltonians, which describe the interaction of quasineutral particles (the bound states of charged fermions) with electromagnetic field, the elementary particles with bound states, and also the bound states with bound states. On the basis of these Hamiltonians one can study such phenomena as Bose-Einstein condensation in a gas of excited atoms, the interaction of condensates with an electromagnetic field in Bose and Fermi systems. These Hamiltonians can be also the basis of the kinetic theory for systems with bound states of particles.

Finally we would like to stress that the developed method can be easily generalized to the case of bound states consisting of more than two particles. The generalization of the offered method for describing the systems with bound states of bosons and also of bosons and fermions taking into account the spin of particles can be also performed without principal difficulties.

\section{Acknowledgments}

The authors would like to acknowledge the financial support of INTAS (project 00-00577) and the Russian Fund of Fundamental Researches (project 03-02-17695). The authors thank A.S. Peletminskii for useful discussions and for his help in preparing of the paper.

${ }^{1}$ S. Chu, Rev. Mod. Phys. 70, 685 (1998).

${ }^{2}$ C.N. Cohen-Tannoudji, Rev. Mod. Phys. 70, 707 (1998).

${ }^{3}$ W.D. Phillips, Rev. Mod. Phys. 70, 721 (1998).

${ }^{4}$ M.H. Anderson et al., Science, 269, 198 (1995).

${ }^{5}$ A.I. Akhiezer, V.B. Berestetskii, Quantum Electrodynamics (Interscience Publisher, New York,1965).

${ }^{6}$ L. Landau, E. Lifshitz, Quantum Mechanics (Pergamon Press, New York,1967).

${ }^{7}$ A.S. Davydov, Quantum Mechanics (Pergamon Press, New York,1976).

${ }^{8}$ A.I. Akhiezer, S.V. Peletminskii, Methods of Statistical Physics (Pergamon Press, Oxford, 1981).

${ }^{9}$ D. Jackson, Fourier series and orthogonal polinomials (The Carus Mathematical Monographs, Number Six, Minnesota, 1941).

${ }^{10}$ For Coulomb's interaction the inequality $R>>r_{0}$ is equivalent to $\mathcal{E}<<\varepsilon_{0}$ because of $r_{0}=\frac{n^{2} \bar{h}^{2}}{m e^{2}}, \varepsilon_{0}=\frac{m e^{4}}{2 n^{2} h^{2}}, n \sim 1$ 